DOE/BG/01569-15

\title{
Western Gas Sands Project Status Report
}

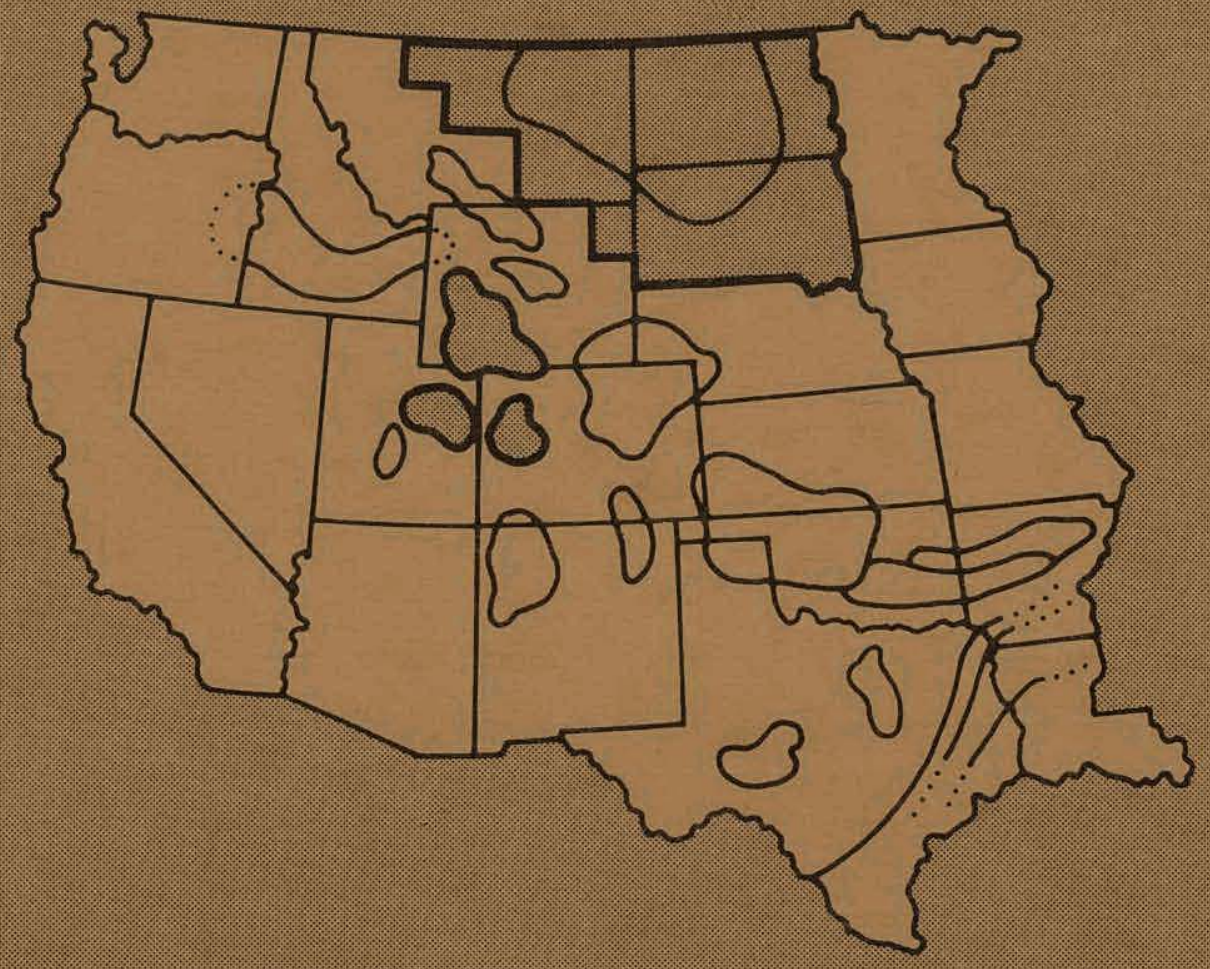

1 September 1979 - 30 September 1979

Prepared for

U.S. Department of Energy

Bartlesville Energy Technology Center

Charles H. Atkinson

Project Manager

Compiled by CER Corporation

Las Vegas, Nevada

Contract DE-AC08-79 BG01569 


\section{DISCLAIMER}

This report was prepared as an account of work sponsored by an agency of the United States Government. Neither the United States Government nor any agency Thereof, nor any of their employees, makes any warranty, express or implied, or assumes any legal liability or responsibility for the accuracy, completeness, or usefulness of any information, apparatus, product, or process disclosed, or represents that its use would not infringe privately owned rights. Reference herein to any specific commercial product, process, or service by trade name, trademark, manufacturer, or otherwise does not necessarily constitute or imply its endorsement, recommendation, or favoring by the United States Government or any agency thereof. The views and opinions of authors expressed herein do not necessarily state or reflect those of the United States Government or any agency thereof. 


\section{DISCLAIMER}

Portions of this document may be illegible in electronic image products. Images are produced from the best available original document. 
This report was prepared as an account of work sponsored by the United States Government. Neither the United States nor the United States DOE, nor any of their employees, nor any of their contractors, subcontractors, or their employees, makes any warranty, express or implied, or assumes any legal liability or responsibility for the accuracy, completeness, or usefulness of any information, apparatus, product or process disclosed, or represents that its use would not infringe privately owned rights.

Available from the National Technical Information Service, U. S. Department of Commerce, Springfield, Virginia 22161.

NATIONAL TECHNICAL INFORMATION SERVICE PAPER COPY PRICES EFFECTIVE JANUARY 1, 1979

$\begin{array}{cc}\begin{array}{c}\text { Page } \\ \text { Range }\end{array} & \begin{array}{c}\text { Domestic } \\ \text { Price }\end{array} \\ & \\ 001-025 & \$ 4.00 \\ 026-050 & \$ 4.50 \\ 051-075 & \$ 5.25 \\ 076-100 & \$ 6.00\end{array}$

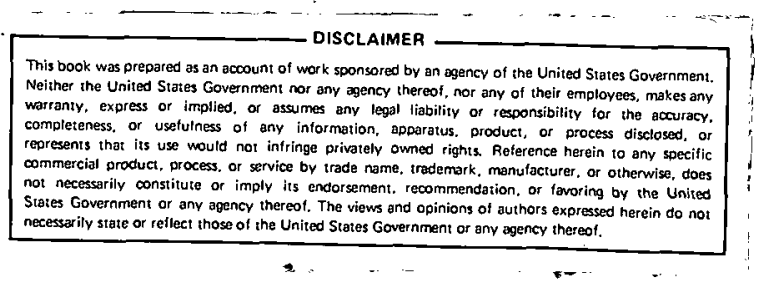




\section{CONTENTS}

Page

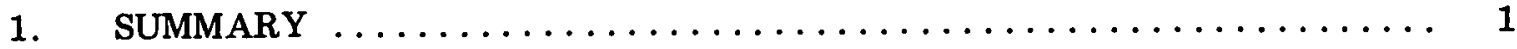

2. PROJECT MANAGEMENT $\ldots \ldots \ldots \ldots \ldots \ldots \ldots \ldots \ldots \ldots \ldots \ldots \ldots \ldots \ldots \ldots$

2.1 Technical Monitoring and Evaluation $\ldots \ldots \ldots \ldots \ldots \ldots \ldots \ldots \ldots$

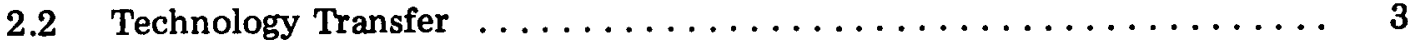

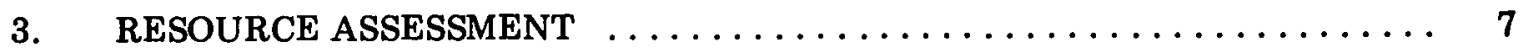

3.1 U.S. Geological Survey Activities $\ldots \ldots \ldots \ldots \ldots \ldots \ldots \ldots \ldots \ldots \ldots$

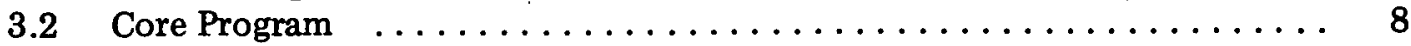

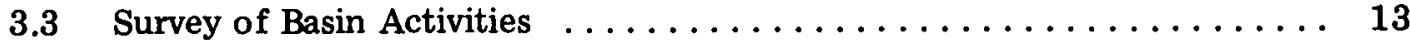

3.4 C K GeoEnergy Corporation $\ldots \ldots \ldots \ldots \ldots \ldots \ldots \ldots \ldots \ldots \ldots \ldots \ldots$

4. RESEARCH AND DEVELOPMENT BY ENERGY TECHNOLOGY CENTERS AND NATIONAL LABORATORIES $\ldots \ldots \ldots \ldots \ldots \ldots \ldots \ldots$

4.1 Bartlesville Energy Technology Center $\ldots \ldots \ldots \ldots \ldots \ldots \ldots \ldots \ldots \ldots$

4.2 Gas Research Institute . . . . . . . . . . . . . . . . . 40

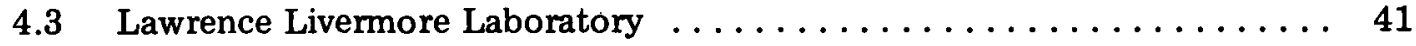

4.4 Los Alamos Scientific Laboratory $\ldots \ldots \ldots \ldots \ldots \ldots \ldots \ldots \ldots \ldots \ldots$

4.5 Sandia Laboratories $\ldots \ldots \ldots \ldots \ldots \ldots \ldots \ldots \ldots \ldots \ldots \ldots \ldots \ldots . \ldots$

5. FIELD TESTS AND DEMONSTRATIONS $\ldots \ldots \ldots \ldots \ldots \ldots \ldots \ldots \ldots$

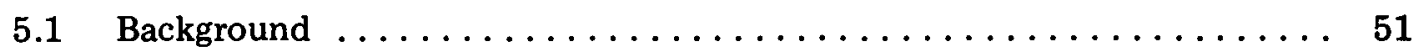

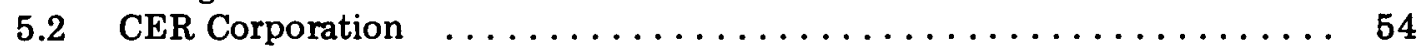

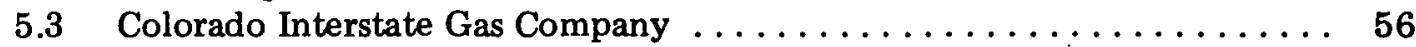

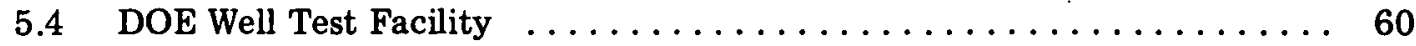

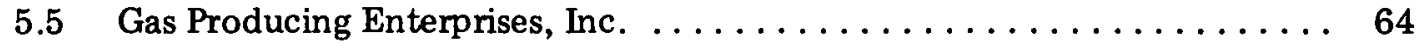

5.6 Mitchell Energy Corporation $\ldots \ldots \ldots \ldots \ldots \ldots \ldots \ldots \ldots \ldots \ldots \ldots$

5.7 Mobil Research and Development Corporation $\ldots \ldots \ldots \ldots \ldots \ldots \ldots \ldots$

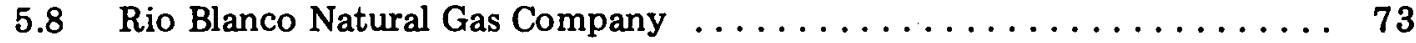

5.9 Sandia Laboratories-Mineback $\ldots \ldots \ldots \ldots \ldots \ldots \ldots \ldots \ldots \ldots \ldots$ 


\section{FIGURES}

Page

Figure $3-1$ Milestone Chart - USGS $\ldots \ldots \ldots \ldots \ldots \ldots \ldots \ldots \ldots \ldots \ldots$

Figure 3-2 Composite Plot of Formation Resistivity Index

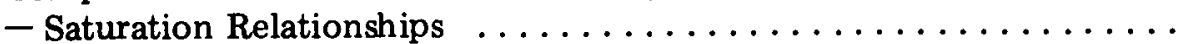

Figure 3-3 Greater Green River Basin Showing Wells of Interest

and USGS Designated Core Areas $\ldots \ldots \ldots \ldots \ldots \ldots \ldots \ldots \ldots \ldots$

Figure 3-4 Northern Great Plains Province Showing Wells of

Interest and USGS Designated Core Areas . . . . . . . . . . 18

Figure 3-5 Piceance Basin Showing Wells of Interest and USGS Designated Core Areas $\ldots \ldots \ldots \ldots \ldots \ldots \ldots \ldots \ldots$

Figure 3-6 Uinta Basin Showing Wells of Interest and USGS Designated Core Areas $\ldots \ldots \ldots \ldots \ldots \ldots \ldots \ldots \ldots \ldots$

Figure 4-1 Proppant Embedment Apparatus $\ldots \ldots \ldots \ldots \ldots \ldots \ldots \ldots \ldots \ldots \ldots$

Figure 4-2 Percent Closure vs. Applied Pressure for 100 percent Coverage $\ldots \ldots \ldots .32$

Figure 4-3 Percent Closure vs. Applied Pressure for 75 percent Coverage $\ldots \ldots \ldots . .33$

Figure 4-4 Percent Closure vs. Applied Pressure for 50 percent Coverage $\ldots \ldots \ldots \ldots 34$

Figure 4-5 Milestone Chart - BETC $\ldots \ldots \ldots \ldots \ldots \ldots \ldots \ldots \ldots \ldots \ldots \ldots$

Figure 4-6 Geometry of Time Dependent Crack Problem $\ldots \ldots \ldots \ldots \ldots \ldots \ldots \ldots$

Figure 4-7 Displacement in Y Direction of Point $\mathrm{x}=.08 \mathrm{c}$ $\mathrm{Y}=.01 \mathrm{c}$. The Elastic Constant for Material 1 is $30 \mathrm{GPa} \ldots \ldots \ldots 42$

Figure 4-8 Geometry of a Fracture near a Frictional Interface $\ldots \ldots \ldots \ldots \ldots \ldots \ldots$

Figure 4-9 Variation in Mode I Stress Intensity Factor as Crack Approaches a Frictional Interface for Variation in Frictional Stress Along the Interface . . . . . 43

Figure 4-10 Milestone Chart - LLL $\ldots \ldots \ldots \ldots \ldots \ldots \ldots \ldots \ldots \ldots \ldots \ldots \ldots$

Figure 4-11 Milestone Chart - LASL $\ldots \ldots \ldots \ldots \ldots \ldots \ldots \ldots \ldots \ldots \ldots \ldots \ldots$

Figure $4-12$ Milestone Chart - Sandia $\ldots \ldots \ldots \ldots \ldots \ldots \ldots \ldots \ldots \ldots \ldots \ldots$ 
Figure 5-1 Production and Injection Volumes and Associated Pressure

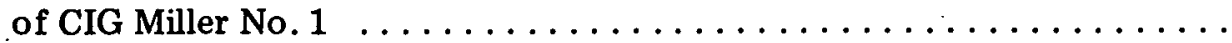

Figure 5-2 Production and Injection Volumes and Associated Pressure of CIG Sprague No. 1 Well $\ldots \ldots \ldots \ldots \ldots \ldots \ldots \ldots \ldots \ldots \ldots$

Figure 5-3 Horner Plot for September 13, BHP Test, RBNG-397-1 Gov't $\ldots \ldots \ldots 62$

Figure 5-4 Flow Rate Performance of Natural Buttes No. 9 Well $\ldots \ldots \ldots \ldots \ldots$

Figure 5-5 Flow Rate Performance of Natural Buttes No. 14 Well $\ldots \ldots \ldots \ldots \ldots 6$

Figure 5-6 Flow Rate Performance of Natural Buttes No. 18 Well $\ldots \ldots \ldots \ldots 6$

Figure 5-7 Flow Rate Performance of Natural Buttes No. 19 Well $\ldots \ldots \ldots \ldots \ldots 6$

Figure 5-8 Flow Rate Performance of Natural Buttes No. 20 Well $\ldots \ldots \ldots \ldots \ldots 6$

Figure 5-9 Flow Rate Performance of Natural Buttes No. 22 Well $\ldots \ldots \ldots \ldots \ldots$

Figure 5-10 Location of CFE Fracture Zones and Mineback $\ldots \ldots \ldots \ldots \ldots \ldots$

Figure $5-11$ Milestone Chart - Sandia Mineback $\ldots \ldots \ldots \ldots \ldots \ldots \ldots \ldots$ 


\section{TABLES}

Page

Table 3-1 Identification and Description of Samples $\ldots \ldots \ldots \ldots \ldots \ldots \ldots$

Table $3-2 \quad$ Acoustic Velocity Data $\ldots \ldots \ldots \ldots \ldots \ldots \ldots \ldots \ldots \ldots \ldots \ldots$

Table 3-3 Air-Brine Capillary Pressure Data $\ldots \ldots \ldots \ldots \ldots \ldots \ldots \ldots \ldots$

Table 3-4 Formation Factor and Resistivity Index Data $\ldots \ldots \ldots \ldots \ldots \ldots$

Table 3-5 Overburden Formation/Factor Data $\ldots \ldots \ldots \ldots \ldots \ldots \ldots \ldots$

Table 3-6 Summary of Wells - Greater Green River Basin $\ldots \ldots \ldots \ldots \ldots \ldots$

Table 3-7 Summary of Wells - Northern Great Plains Province $\ldots \ldots \ldots \ldots \ldots$

Table 3-8 Summary of Wells - Piceance Basin $\ldots \ldots \ldots \ldots \ldots \ldots \ldots \ldots$

Table $3-9$ Summary of Wells - Uinta Basin $\ldots \ldots \ldots \ldots \ldots \ldots \ldots \ldots \ldots \ldots \ldots$

Table 4-1 Field Test Results, Single Piece Stratapax® Core Bit . . . . . . . . . 24

Table $4-2 \quad$ Gas Permeability Measurements $\ldots \ldots \ldots \ldots \ldots \ldots \ldots \ldots \ldots$

Table $4-3 \quad$ Gas Permeability Measurements $\ldots \ldots \ldots \ldots \ldots \ldots \ldots \ldots \ldots$

Table 4-4 Size Distribution of 12-20 Mesh Sintered Bauxite Proppant $\ldots . \ldots \ldots 30$

Table 5-1 MHF Contract Location and Frac Data $\ldots \ldots \ldots \ldots \ldots \ldots \ldots$

Table 5-2 BHP Buildup Test Commencing September 13, RBNG 397-19-1 Gov't . . . . . . . . . . . . . . . . . . . . 61 


\section{SUMMARY}

This edition of the WGSP Status Report summarizes September, 1979, progress of the government-sponsored projects directed toward increasing gas production from the lowpermeability gas sands of the western United States. Background information is provided in the September, 1977, WGSP Status Report, NVO/0655-100.

DOE's WGSP display was exhibited at the International Petroleum Exposition Energy '79 meeting in Tulsa, Oklahoma, and at the annual meeting of the Society of Petroleum Engineers of AIME in Las Vegas, Nevada, during the month of September.

Projects of the National Laboratories and Energy Technology Centers progressed on schedule in September. Bartlesville Energy Technology Center continued work on rock-fluid interaction and advanced logging techniques. Lawrence Livermore Laboratory continued experimental and theoretical work on hydraulic fracturing mechanics and analysis of well test data. Los Alamos Scientific Laboratory continued work on permeability and porosity determination of core samples and geological support studies. Sandia Laboratories continued work on their EGR Instrumentation and Diagnostic Program.

Cyclic gas injection continued at Colorado Interstate Gas Company's Miller No. 1 and Sprague No. 1 wells. The DOE Well Test Facility is continuing to provide technical support to the Gas Research Insitute/Rio Blanco Natural Gas MHF experiment. The Gas Producing Enterprises, Inc. Natural Buttes Unit wells continued to flow to sales. The Mitchell Energy Corporation Muse-Duke No. 1 was opened after a 28-day shut-in period. The hydraulic fracturing containment experiment continued for the Sandia-mineback program. 
THIS PAGE

\section{WAS INTENTIONALLY LEFT BLANK}




\section{PROJECT MANAGEMENT}

\subsection{TECHNICAL MONITORING AND EV ALUATION}

C. H. Atkinson (DOE) and R. L. Mann (CER Corporation) participated in a workshop on unconventional gas resources at Los Alamos Scientific Laboratory, New Mexico, September 5-6.

G. R. Luetkehans, J. Baltes (CER Corporation), W. Bailey and W. Murphy (TRW) attended the International Petroleum Exposition Energy '79 meeting in Tulsa, Oklahoma, September 10-13. DOE's WGSP display was set up and manned for exhibition.

C. H. Atkinson (DOE), E. Evered, D. Bleakly, N. Newman, G. Kukal (CER Corporation), W. Murphy, and W. Bailey (TRW) attended the Society of Petroleum Engineers of AIME fall meeting in Las Vegas, Nevada, September 24-26. The WGSP display was exhibited at this meeting.

L. F. Elkins (Sohio Petroleum Company), J. L. Fitch (Mobil Research and Development Corporation), R. J. Saucier (Shell Development Company), R. W. Veatch (Amoco Production Company) and D. A. Northrop (Sandia Laboratories) met with C. H. Atkinson (DOE), R. L. Mann and G. R. Luetkehans (CER Corporation) in Las Vegas, Nevada, September 24, to discuss the WGSP Multi-well Experiment. A proposed location for the experiment was reviewed.

A meeting was held at CER offices in Las Vegas, Nevada, with Sandia Laboratories on September 27 to discuss the Multi-well Experiment arrangements and funds.

C. H. Atkinson (DOE) met with DOE management personnel from BETC, METC and DOE/ HQ in Las Vegas, Nevada, September 26 and 27, regarding the WGSP and proposed Federal Energy Regulatory Commission incentives for production from tight gas sands.

\subsection{TECHNOLOGY TRANSFER}

\subsubsection{Documentation Reports}

The WGSP Financial Supplements for August, 1979, and September, 1979, have been distributed. Work is continuing on the DOE Semi-Annual Report for the Unconventional Gas Recovery Program (period ending September 30,1979) and WGSP Third Quarterly Basin Activities Report, 1979. The WGSP Second Quarterly Basin Activities Report, 1979, has been distributed. The WGSP Project Plan, FY 1980, has been completed. The DOE Well Test Facility Operations Manual and a report on the WGSP Logging Program have been updated. 


\subsubsection{Project Data Bank}

Work continued on the WGSP bibliography.

\subsubsection{Articles and Publications}

The following articles and publications relate to the WGSP and are included in the bibliography. Abstracts are included when available.

\section{The Effects of Mechanical and Frictional Rock Properties On Hydraulic Fracture Growth Near Unbonded Interfaces by Gordon $\mathrm{D}$. Anderson \\ Lawrence Livermore Laboratory}

Experiments are being performed to study the growth of hydraulically driven cracks in the vicinity of an unbonded interface in rocks. The materials on either side of the interface may be the same or different. The materials used in these studies are Nugget sandstone from Utah (3-6 percent porosity) and Indiana limestone (12-15 percent porosity). The fracturing fluid is oil which is injected into the test specimens through high pressure steel tubing. Blocks of the rock materials which are to be studied are held adjacent to one another in a hydraulic press so that a normal stress is set up across their mutual interface. A hydraulically driven crack is initiated in one of the blocks. It is found that the major parameters which affect hydraulic crack penetration into the adjacent block are the relative values of the mechanical strength and moduli of the rocks on either side of the interface, the finish of the interface surfaces, and the magnitude of the normal stress across the interface. To gain a better understanding of the mechanisms which control the growth of cracks across the interfaces, experiments are being performed to study the frictional properties of these interface surfaces. The experiments involve the simultaneous measurement of the normal force across the interface and the applied force necessary to overcome friction to initiate displacement. These experiments indicate that in addition to the normal force, the frictional force depends upon the surface finish and the presence of water in the rock.

\section{Theoretical and Experimental Analyses of Hydraulic Fracturing and Some Reservoir Response to the Stimulation by M. E. Hanson, G. D. Anderson, R. J. Shaffer, L. D. Thorson and D. N. Montan Lawrence Livermore Laboratory}

We are conducting a joint theoretical/experimental research program on hydraulic fracturing. Newly developed numerical models have been applied to analyze some aspects of fracture propagation near well bonded material interfaces to determine if these interfaces can inhibit propagation. Results from these calculations indicate that, for fractures propagating from a lower modulus material toward an interface with a higher modulus material, the stress intensity factor is seen to increase abruptly and arrive at a higher value than in the 
lower modulus material. Conversely, when the fracture is propagating from a lower modulus material toward a higher modulus material, the situation is reversed. The presence of existing fractures near the interface is seen to significantly reduce the effects of these phenomena.

Small scale laboratory experiments are being performed to study the growth of hydraulically driven cracks in the vicinity of unbonded interfaces in rocks. Blocks of the materials being studied are held adjacent to one another under a static load. A hydraulically driven crack is initiated in one of the blocks. It is found that for blocks of the same material that penetration of the crack into the adjacent block is controlled by the normal stress across the interface and the finish of the interface surfaces. Experiments are performed to measure the frictional properties of the interfaces to better understand the mechanism of crack growth across the interface.

Well pressure test data from a MHF stimulated well in the Piceance Basin was analyzed. Analysis of that data indicated that the length of the fracture in the productive rock was of the order of the lens size. Additionally, the analysis showed that the fracture had a finite conductivity or there was fluid damage to the fracture faces, but it was impossible to uniquely determine if either mechanism was dominant. 
THIS PAGE

WAS INTENTIONALLY

LEFT BLANK 


\section{RESOURCE ASSESSMENT}

\subsection{U.S. GEOLOGICAL SURVEY ACTIVITIES}

\subsubsection{Uinta-Piceance Basins}

A paper which describes and discusses the stratigraphy, mineralogy and diagenesis of the uppermost Cretaceous rocks from the surface of the Piceance Basin and the subsurface of the Rio Blanco area, Colorado, is being written by P. Hansley and R. Johnson. These Cretaceous rocks contain many of the basin's low-permeability reservoirs.

A map illustrating structure contours on the top of the Cretaceous horizons for the entire Piceance Basin is being prepared.

Additional core (provided by Amoco Production Company) of lower Tertiary hydrocarbonbearing rocks from the south central Uinta Basin, Utah, was examined. Analyses of mineralogy and diagenesis of the unconventional reservoirs cored in the area have begun.

Several hundred feet of core from the Chapita Wells area of the Uinta Basin were examined. The units are primarily early Tertiary in age.

\subsubsection{Greater Green River Basin}

Work on stratigraphic analysis of the Greater Green River Basin continued.

Description and sampling of core from the Inexco (Belco) No. 1-A WASP well, Sec. 28, T36N, R112W, Sublette County, Wyoming, was completed.

Two cross sections from the Washakie Basin by T. F. Tyler have been completed and are in review.

L. W. Kiteley submitted a cross section from the Sand Wash Basin for review.

B. E. Law presented a paper at the 5th Annual DOE Enhanced Oil \& Gas Recovery Symposium entitled "Preliminary results of organic maturation, temperature, and pressure studies in the Pacific Creek area, Sublette County, Wyoming," by B. E. Law, C. W. Spencer, and N. H. Bostick.

\subsubsection{Northern Great Plains Province}

A paper on petrology of the Eagle Sandstone in the Bearpaw Mountains area, which is intended as a USGS Bulletin, was revised. 
A USGS Open-File Report on the petrology of Eagle Sandstone equivalents in the J. J. C. Paine well, north-central Montana, was prepared.

D. D. Rice attended a workshop on Alternative Gas Sources at LASL.

A paper on the origin, accumulation and resource potential of biogenic gas is being prepared.

A resource estimate of the study area is being prepared for the National Petroleum Council.

\subsubsection{Schedule Status}

Figure 3-1 is a milestone chart depicting the status of all USGS projects within the WGSP.

\subsection{CORE PROGRAM}

In the Greater Green River Basin, Colorado Interstate Gas Exploration was contacted concerning the coring of their Haystack Unit well No. 2-27-14-96 in Sweetwater County, Wyoming. Also in Sweetwater County, Pacific Transmission Supply Company was contacted in regards to coring their well No. 3-10A Federal in the Red Desert sub-basin. Work also continued on a Greater Green River Basin study.

In the Piceance Basin, coring of the Mancos "B" section of the Pacific Transmission Supply well No. 22-12 Federal, Sec. 12, T1N, R99W, Rio Blanco County, Colorado, was delayed until October. Although originally scheduled for mid-September, coring was delayed due to encountering an unexpectedly thick Mesaverde interval. Core depth was originally projected to be about $10,500 \mathrm{ft}$, but later revised to $11,500 \mathrm{ft}$. Also in the Piceance Basin, Exxon's Vega Unit No. 4 well, Mesa County, was under consideration as a coring location. After arrangements had been made, however, the proposal was rejected by the operator.

Discussion continued concerning Mapco RBU No. $11-17 \mathrm{~F}$ well in the Uinta Basin, Sec. 17, T10S, R20E, Uintah County, Utah. Both Wasatch and Mesaverde coring was discussed. Spud in is expected around mid-October, with coring being planned about two weeks later.

Core Laboratories, Inc. performed various routine and special core analysis tests on core sample from the Twin Arrow C\&K No. 4-14 well, Rio Blanco County, Colorado. These tests included acoustic velocity measurements, capillary pressure tests, formation resistivity factor measurements at various overburden pressures, and formation resistivity index measurements. The core samples used are described and identified in Table 3-1.

Three full-diameter cores from $1,042 \mathrm{ft}, 1,143 \mathrm{ft}$ and $1,144 \mathrm{ft}$ were used in this study. Core plugs, 1-in. in diameter, were obtained in the horizontal direction from the depths $1,042 \mathrm{ft}$ and $1,143 \mathrm{ft}$ and in the vertical direction from the depths $1,143 \mathrm{ft}$ and 1,144 ft. Each core plug was voided of hydrocarbons with an alternate injection of toluene and acetone, leached of salt with methyl alcohol, and then dried. Air permeabilities and Boyle's law porosities were determined on the cleaned and dried core plugs. Each core plug was evacuated and saturated with a brine containing $12,000 \mathrm{ppm}$ total dissolved solids. 

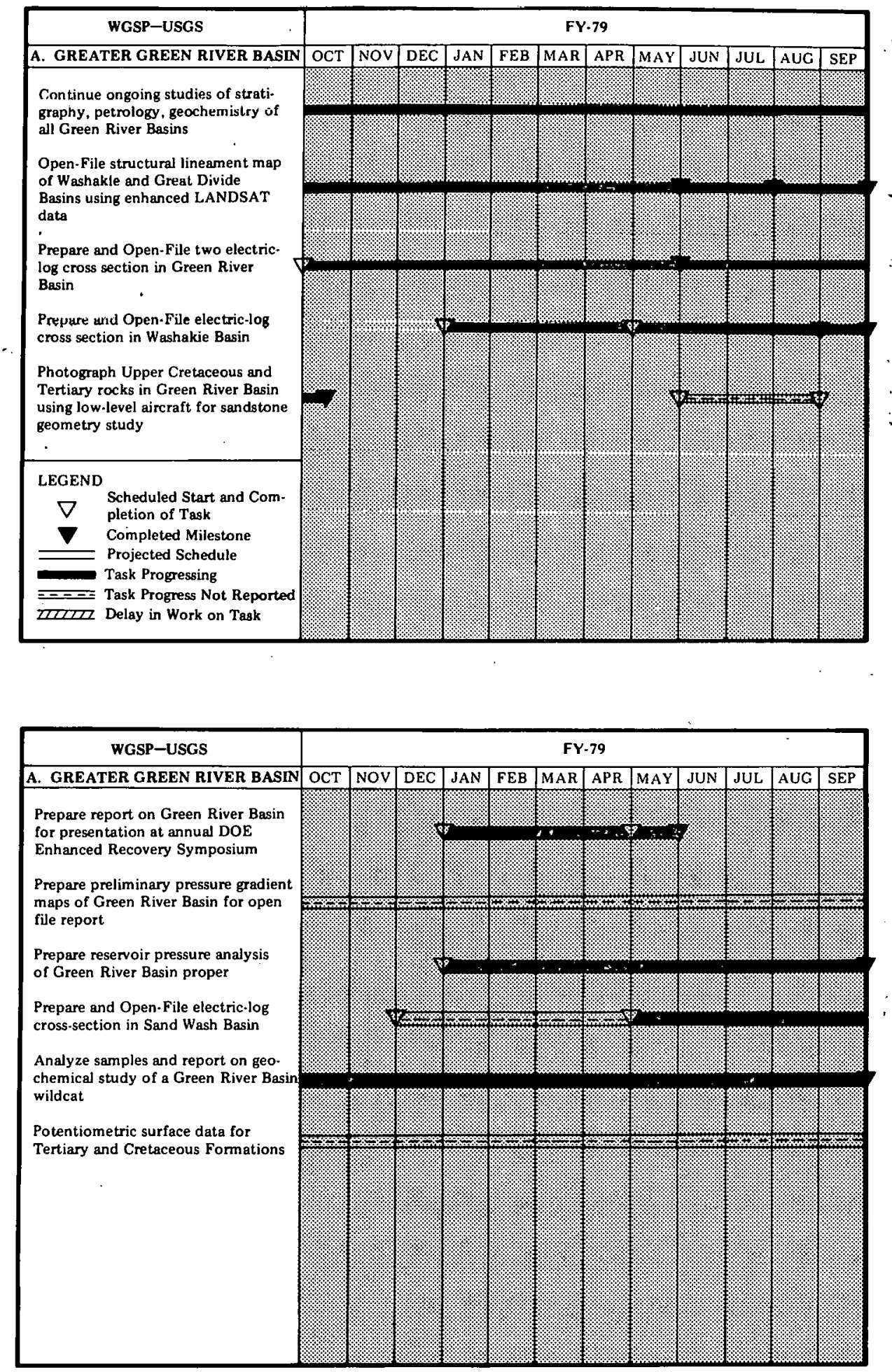

Figure 3-1 Milestone Chart-USGS 

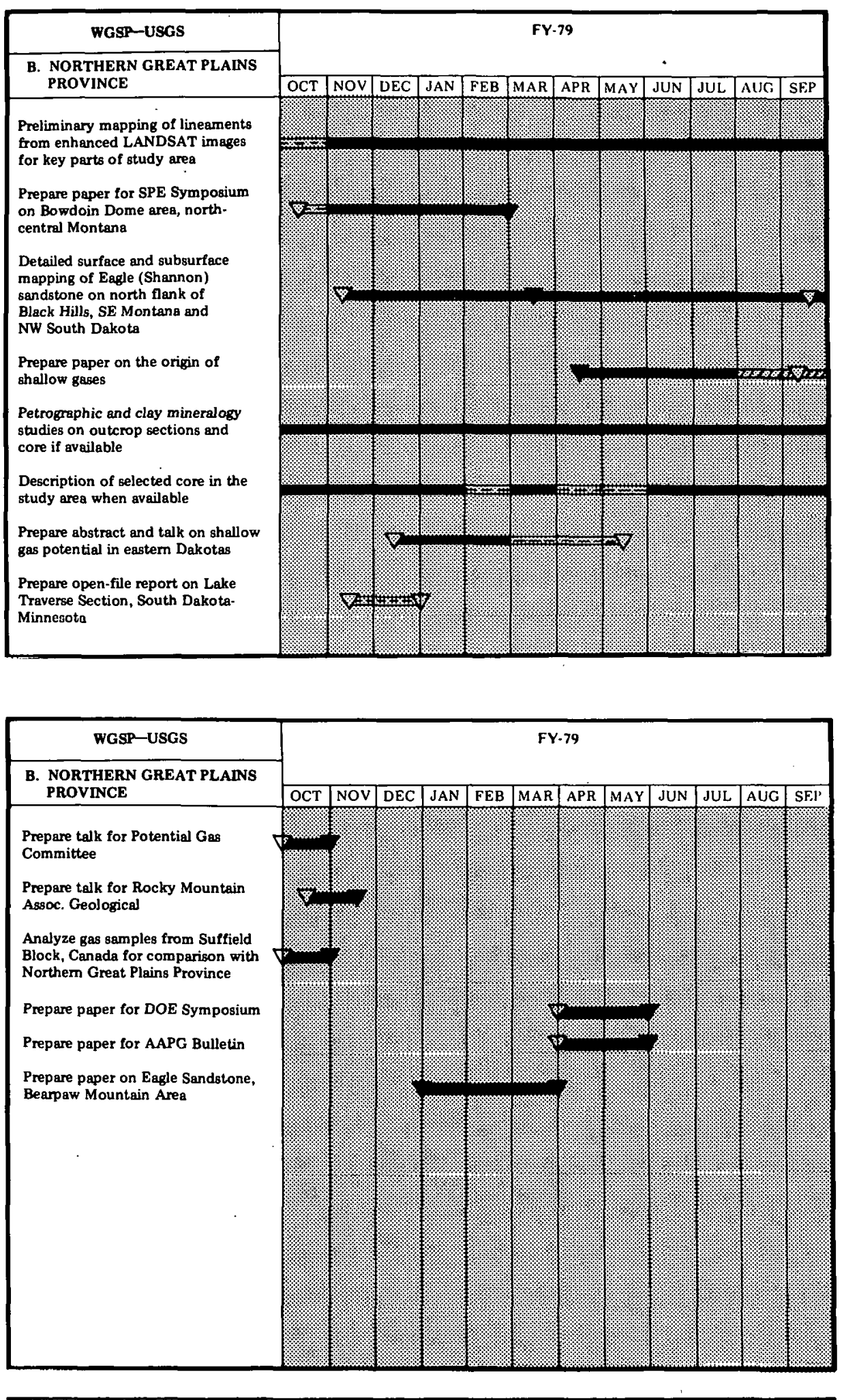

Figure 3-1 Continued 

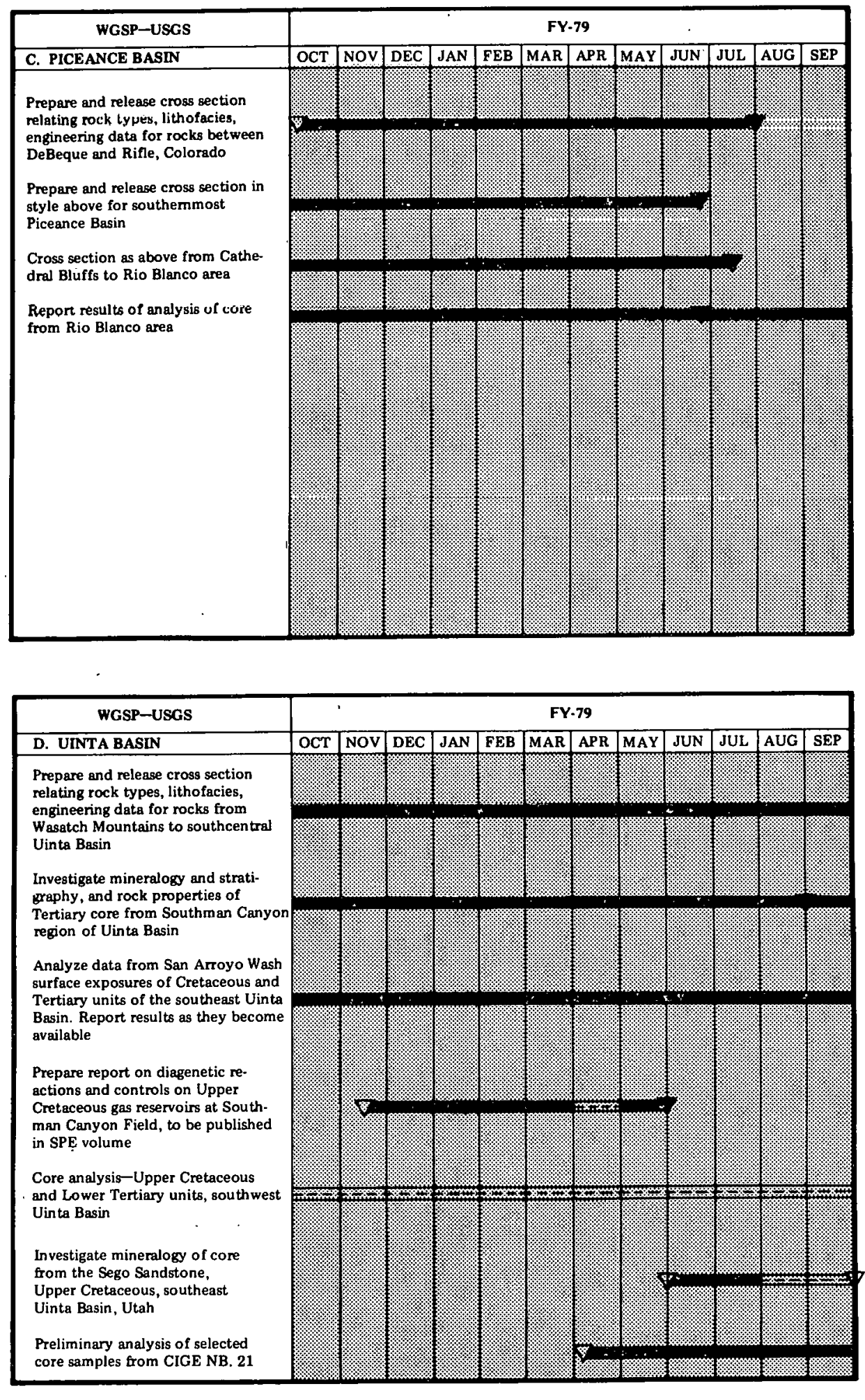

Figure 3-1 Continued 
Table 3-1 Identification and Description of Samples

\begin{tabular}{lcc}
\hline $\begin{array}{l}\text { Sample } \\
\text { Number }\end{array}$ & Depth,ft & Lithological Description \\
\hline 1 & 1,042 & Ss, bf, v/fn fn gr, well indurated, sl/slty \\
2 & 1,143 & Ss, bf, v/fn fn gr, well indurated, sl/slty \\
2V & 1,143 & Ss, bf, v/fn fn gr, well indurated, sl/slty \\
$3 V$ & 1,144 & Ss, bf, v/fn fn gr, well indurated, sl/slty \\
\hline
\end{tabular}

Table 3-2 Acoustic Velocity Data

\begin{tabular}{lcc}
\hline Sample Number: & $2 \mathrm{~V}$ & $3 \mathrm{~V}$ \\
Porosity, Percent: & $\mathbf{1 8 . 1}$ & $\mathbf{1 8 . 7}$ \\
\hline $\begin{array}{l}\text { Effective Overburden } \\
\text { Pressure, psi }\end{array}$ & \multicolumn{3}{c}{} \\
\hline 400 & Transit Time, Micro-Seconds/ft \\
800 & 96.19 & 101.71 \\
1,200 & 91.23 & 95.17 \\
1,600 & 87.99 & 90.97 \\
2,000 & 85.77 & 88.28 \\
\hline
\end{tabular}

Table 3-3 Air-Brine Capillary Pressure Data

\begin{tabular}{cccccccccccc}
\hline & \multicolumn{2}{c}{ Pressure, psi: } & 1 & 2 & 4 & 8 & 15 & 35 & 200 & $350 *$ \\
\hline $\begin{array}{c}\text { Sample } \\
\text { Number }\end{array}$ & $\begin{array}{c}\text { Permeability } \\
\text { md }\end{array}$ & $\begin{array}{c}\text { Porosity, } \\
\text { Percent }\end{array}$ & & \multicolumn{1}{c}{ Brine Saturation, } \\
\hline 1 & 130 & 22.8 & 100 & 96.7 & 52.6 & 40.8 & 340.0 & 26.9 & 26.5 & 19.7 \\
2 & 24 & 18.2 & 100 & 100.0 & 91.0 & 69.4 & 54.2 & 42.3 & 38.2 & 26.4 \\
\hline
\end{tabular}

*Equivalent pressure from centrifuge (air-brine system) 
Direct measurements of transit times were performed on the two vertical core plugs using effective overburden pressures ranging from 400 psi to 2,000 psi. These data are presented in Table 3-2.

With the exception of a final high-speed centrifuge point, eight-point capillary pressure tests were performed on the two core plugs obtained in the horizontal direction utilizing an air-brine system and a porous-plate cell. The results of these tests are presented in Table 3-3.

Prior to performing the capillary pressure tests, the electrical resistivities of the brine and the two brine-saturated core plugs were measured. These measurements were repeated over a period of several days until the electrical resistivities stabilized, indicating that ionic equilibrium within the core plugs had been attained. Each core plug was desaturated during the capillary pressure tests and the electrical resistivities were measured at each equilibrium saturation attained. The results of these data are presented in Table 3-4. Using Archie's equation, a cementation exponent " $m$ " of 1.74 is calculated. The formation resistivity index saturation relationships yield calculated saturation exponents " $n$ " of 1.88 and 1.99. A composite plot of the formation resistivity index-saturation relationships is presented in Figure 3-2.

Each core plug used for the capillary pressure and electrical resistivity tests was leached of salt with methyl alcohol, and then dried. The air permeabilities and Boyle's law porosities were again determined on the cleaned and dried core plugs. Each core plug was evacuated and pressuresaturated with a brine containing $12,000 \mathrm{ppm}$ total dissolved solids. The electrical resistivities of the brine and the brine-saturated core plugs were again measured in the manner already described using no overburden pressure. Formation resistivity factors were also measured using effective overburden pressures ranging from $200 \mathrm{psi}$ to $2,000 \mathrm{psi}$. The results of these data are presented in Table 3-5. The formation resistivity factor at 0 psi effective overburden pressure yields a cementation exponent " $\mathrm{m}$ " of 1.79. The cementation exponent increases to 1.93 at an effective overburden pressure of $200 \mathrm{psi}$ and up to 1.98 at an effective overburden pressure of $2,000 \mathrm{psi}$. These data follow a normal trend of increasing formation resistivity factor with increasing effective overburden pressure.

\subsection{SURVEY OF BASIN ACTIVITIES}

\subsubsection{Greater Green River Basin}

In this basin, 7 development wells were completed, with 5 of these wells producing and 2 wells D\&A. One development well abandoned location. Of 3 wildcat wells completed, 1 was producing and 2 were D\&A.

This basin was the largest gas producer of the four primary study areas in the WGSP, with a total of 11,916 MCFD of new gas. Producing horizons included Bear River (5,896 MCFD), Almond (1,387 MCFD) and Lewis (4,633 MCFD).

During September, 30 new wells were staked: 21 development and 9 wildcat wells.

Wells and core areas of interest are summarized in Table 3-6 and are shown in Figure 3-3. 
Table 3-4 Formation Factor and Resistivity Index Data

Resistivity of Saturating Brine, ohm-meters: $\quad 0.4712 @ 72.0^{\circ} \mathrm{F}$.

\begin{tabular}{|c|c|c|c|c|c|}
\hline $\begin{array}{l}\text { Sample } \\
\text { Number }\end{array}$ & $\begin{array}{l}\text { Air Permeability, } \\
\text { md }\end{array}$ & $\begin{array}{l}\text { Porosity, } \\
\text { Percent }\end{array}$ & $\begin{array}{l}\text { Formation } \\
\text { Factor }\end{array}$ & $\begin{array}{l}\text { Brine Saturation, } \\
\text { Percent Pore Space }\end{array}$ & $\begin{array}{l}\text { Resistivity } \\
\text { Index }\end{array}$ \\
\hline 1 & 130 & 22.8 & 12.2 & $\begin{array}{r}100.0 \\
96.7 \\
52.6 \\
40.8 \\
34.0 \\
26.5 \\
19.7\end{array}$ & $\begin{array}{r}1.00 \\
1.10 \\
3.44 \\
5.44 \\
9.10 \\
14.80 \\
20.10\end{array}$ \\
\hline 2 & 24 & 18.2 & 20.4 & $\begin{array}{r}100.0 \\
91.0 \\
69.4 \\
54.2 \\
42.3 \\
38.2 \\
26.4\end{array}$ & $\begin{array}{r}1.00 \\
1.30 \\
2.33 \\
3.79 \\
6.02 \\
6.73 \\
11.00\end{array}$ \\
\hline
\end{tabular}

Table 3-5 Overburden Formation/Factor Data

Saturant: $\quad$ Simulated Formation Brine

Resistivity of Saturant, ohm-meters: 0.418 @77.5 ${ }^{\circ} \mathrm{F}$.

\begin{tabular}{|c|c|c|c|c|c|c|c|c|c|}
\hline \multirow[b]{2}{*}{$\begin{array}{l}\text { Sample } \\
\text { Number }\end{array}$} & \multirow[b]{2}{*}{$\begin{array}{l}\text { Depth, } \\
\text { ft }\end{array}$} & \multirow[b]{2}{*}{$\begin{array}{l}\text { Permeability, } \\
\text { md }\end{array}$} & \multirow[b]{2}{*}{$\begin{array}{l}\text { Porosity, } \\
\text { Percent }\end{array}$} & \multicolumn{6}{|c|}{ Effective Overburden Pressure, psi } \\
\hline & & & & 0.0 & $\begin{array}{l}200 \\
\text { Form: }\end{array}$ & $\begin{array}{c}400 \\
\text { ation }\end{array}$ & $\begin{array}{l}800 \\
\text { Resistivi }\end{array}$ & $\begin{array}{l}1,200 \\
\text { ity Fact }\end{array}$ & 2,000 \\
\hline 1 & 1,042 & 130 & 22.8 & 14.0 & 18.4 & 18.9 & 19.4 & 19.9 & 20.2 \\
\hline 2 & 1,143 & 24 & 18.2 & 21.0 & 25.9 & 26.2 & 26.2 & 26.4 & 27.2 \\
\hline
\end{tabular}




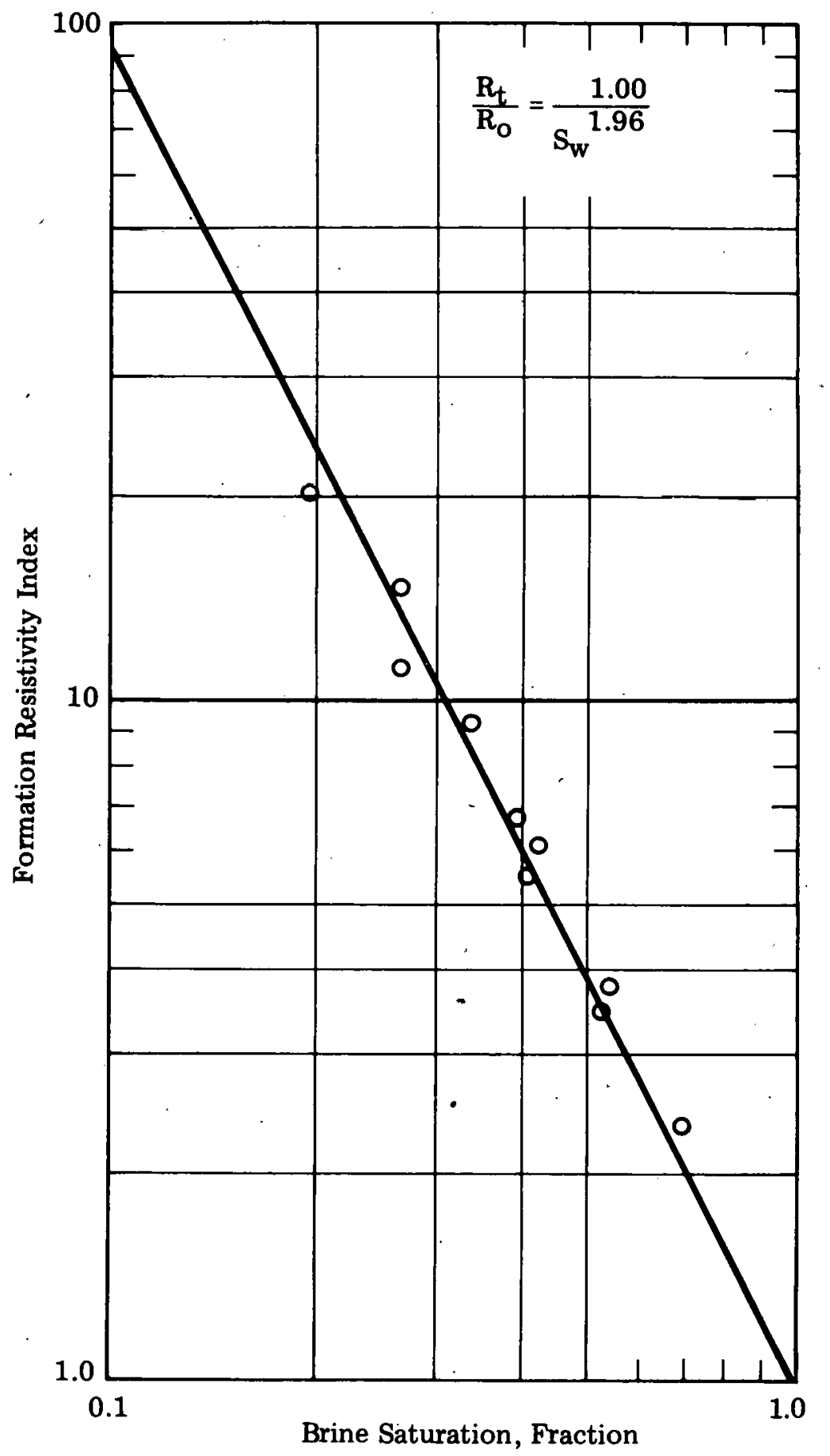

Figure 3-2 Composite Plot of Formation Resistivity Index - Saturation Relationships 


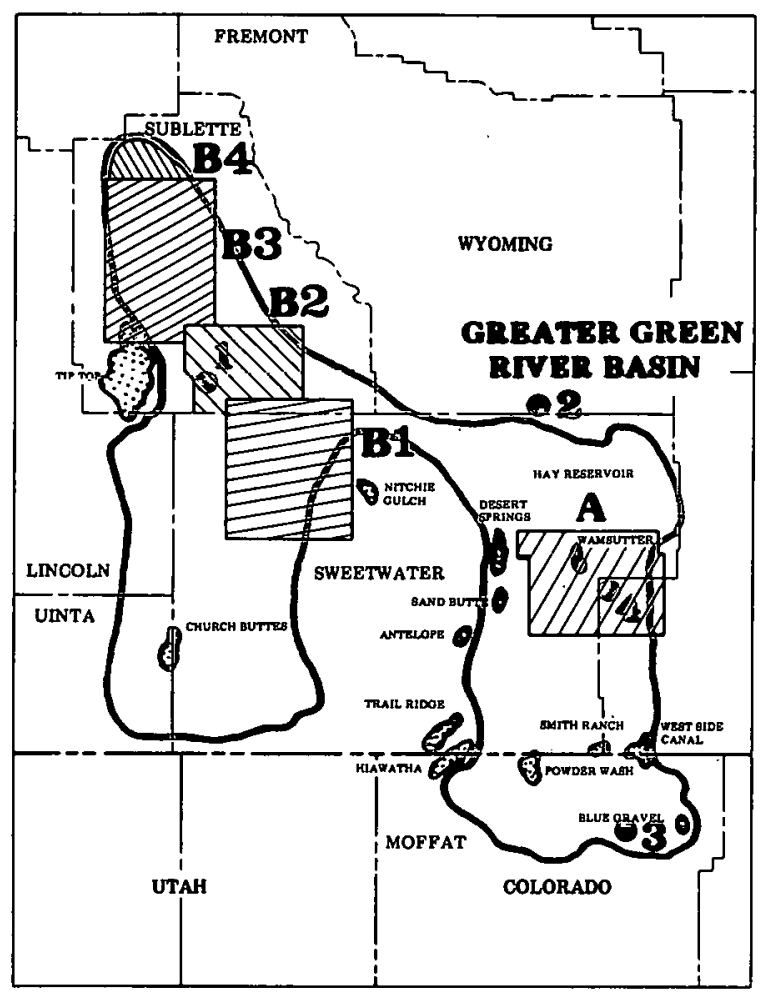

Figure 3-3

Greater Green River Basin Showing Wells of Interest and USGS Desig. nated Core Areas (refer to Table 3.6)

Table 3-6 Summary of Wells - Greater Green River Basin

\begin{tabular}{|c|c|c|c|c|c|c|c|c|}
\hline OPERATOR & $\begin{array}{l}\text { WELL } \\
\text { NAME } \\
\end{array}$ & $\begin{array}{c}\text { MAP } \\
\text { INDEX } \\
\text { NO. } \\
\end{array}$ & $\begin{array}{c}\text { LOCATION } \\
\text { Sec/T/R } \\
\end{array}$ & $\begin{array}{c}\text { HORIZON }^{2} \\
\mathrm{ft}\end{array}$ & $\begin{array}{c}\text { FINAL } \\
\text { TD }\end{array}$ & $\begin{array}{l}\text { FRACTURE } \\
\text { TREATMEN }\end{array}$ & STATUS & $\begin{array}{l}\text { IPF in } \\
\text { MCFD }\end{array}$ \\
\hline $\begin{array}{l}\text { Energetics } \\
\text { Inc. }\end{array}$ & $\begin{array}{l}\text { 10-29 } \\
\text { Federal }\end{array}$ & 1 & $\begin{array}{l}\text { nwnw } 29 / 27 N / \\
111 \text { W } \\
\text { Bird Canyon Field } \\
\text { Sublette Cnty, WY }\end{array}$ & $\begin{array}{l}\text { Béar River } \\
(9,693-9,730)\end{array}$ & $\begin{array}{l}9,925 \\
\text { PB: } \\
9,874\end{array}$ & $\begin{array}{l}64,760 \mathrm{gal} \\
\text { gel, } 79,000 \\
\text { lb sand }\end{array}$ & $\begin{array}{l}\text { Develop. well } \\
\text { comp. 7-16-79, } \\
\text { no cores or tests. } \\
\text { Located in Core } \\
\text { Area B2. }\end{array}$ & 4,400 \\
\hline $\begin{array}{l}\text { Davis } \\
\text { Oll }\end{array}$ & $\begin{array}{l}\text { 3 Picket } \\
\text { Lake Unit }\end{array}$ & 2 & $\begin{array}{l}\text { nesw } 18 / 26 N / 96 W \\
\text { Picket Lake Field } \\
\text { Sweetwater Cnty. } \\
\text { WY }\end{array}$ & $\begin{array}{l}\text { Lewis } \\
(13,716-13,736)\end{array}$ & 14,000 & $\begin{array}{l}166,740 \mathrm{gal} \\
\text { emul, } \\
191,250 \mathrm{lb} \\
\text { sand }\end{array}$ & $\begin{array}{l}\text { Whdcat well } \\
\text { comp. 7-31-79. } \\
\text { Lewis Extension- } \\
\text { Picket Lake. }\end{array}$ & $\mathbf{3 , 1 7 5}$ \\
\hline $\begin{array}{l}\text { Northwest } \\
\text { Exploration }\end{array}$ & 1 Bruder & 3 & $\begin{array}{l}\text { nene } 4 / 9 N / 93 W \\
\text { Great Divide Field } \\
\text { Moffat Cnty, CO }\end{array}$ & $\begin{array}{l}\text { Lewis } \\
(7,626-7,540)\end{array}$ & $\begin{array}{l}7,800 \\
\text { PB: } \\
7,671\end{array}$ & $\begin{array}{l}42,000 \text { gal } \\
\text { emul, } \\
\text { Acidized } \\
\text { w/ } 2,000 \\
\text { gal. }\end{array}$ & $\begin{array}{l}\text { Develop. well } \\
\text { comp. 11-2-78. } \\
\text { No cores or tests. }\end{array}$ & 1,146 \\
\hline Sun Oil & $\begin{array}{l}\text { 1-8 Fair- } \\
\text { Federal }\end{array}$ & 4 & $\begin{array}{l}\text { csw } 8 / 19 N / 92 W \\
\text { Echo Springs } \\
\text { Field } \\
\text { Carbon Cnty, WY }\end{array}$ & $\begin{array}{l}\text { Mesaverde } \\
(10.020)\end{array}$ & & & $\begin{array}{l}\text { Develop. well } \\
\text { reported 9-6-79. } \\
\text { Located in Core } \\
\text { Area A. }\end{array}$ & \\
\hline
\end{tabular}

\footnotetext{
${ }^{1}$ Refer to Figure $3-3$

${ }^{2}$ Horizon - projected depth or producing interval
} 


\subsubsection{Northern Great Plains Province}

During September, 24 development wells were completed in this basin. Fifteen of these were producing and 9 were D\&A. Three wildcat wells were completed, with 1 producing and 2 D\&A.

New gas production totaled 3,632 MCFD from the Eagle, Bow Island and 2nd Bow Island. Seven IPFs were not available from Petroleum Information: 5 from the Eagle horizon, 1 from Bow Island, and 1 from Blackleaf/Bow Island commingled.

Forty new wells were reported in September: 27 development and 13 wildcat wells.

Wells and core areas of interest are summarized in Table 3-7 and are shown on Figure 3-4.

\subsubsection{Piceance Basin}

Of 14 development wells reported completed this month, 13 were producing and 1 was D\&A. One wildcat well was successful.

Producing horizons included the Rollins, Cozzette, Corcoran, Mancos and Mancos B for a total of 10,871 MCFD of new gas.

During the month, 17 new wells were reported: 10 development and 7 wildcat wells.

A summary of wells and core areas of interest is given in Table 3-8 and shown in Figure 3-5.

\subsubsection{Uinta Basin}

During August, 3 development wells were completed, producing a total of 6,547 MCFD from the Wasatch horizon. No wildcat wells were reported completed.

Ten new wells, 7 development and 3 wildcat, were staked during the month.

Wells and core areas of interest are summarized in Table 3-9 and are shown in Figure 3-6.

\subsection{K GEOENERGY}

\subsubsection{Development of Techniques for Optimizing Completion of Western Tight Gas Sands}

During the month of September 1979, the Green River Basin outcróp reconnaissance task was completed and field work was started. Three general areas were selected for outcrop evaluation:

- the Hoback Basin, a northwestern extension of the restricted Green River Basin;

- the Cretaceous Mountain/Hogsback Mountain area, outside of the overthrust belt located on the western edge of the Green River Basin; and 


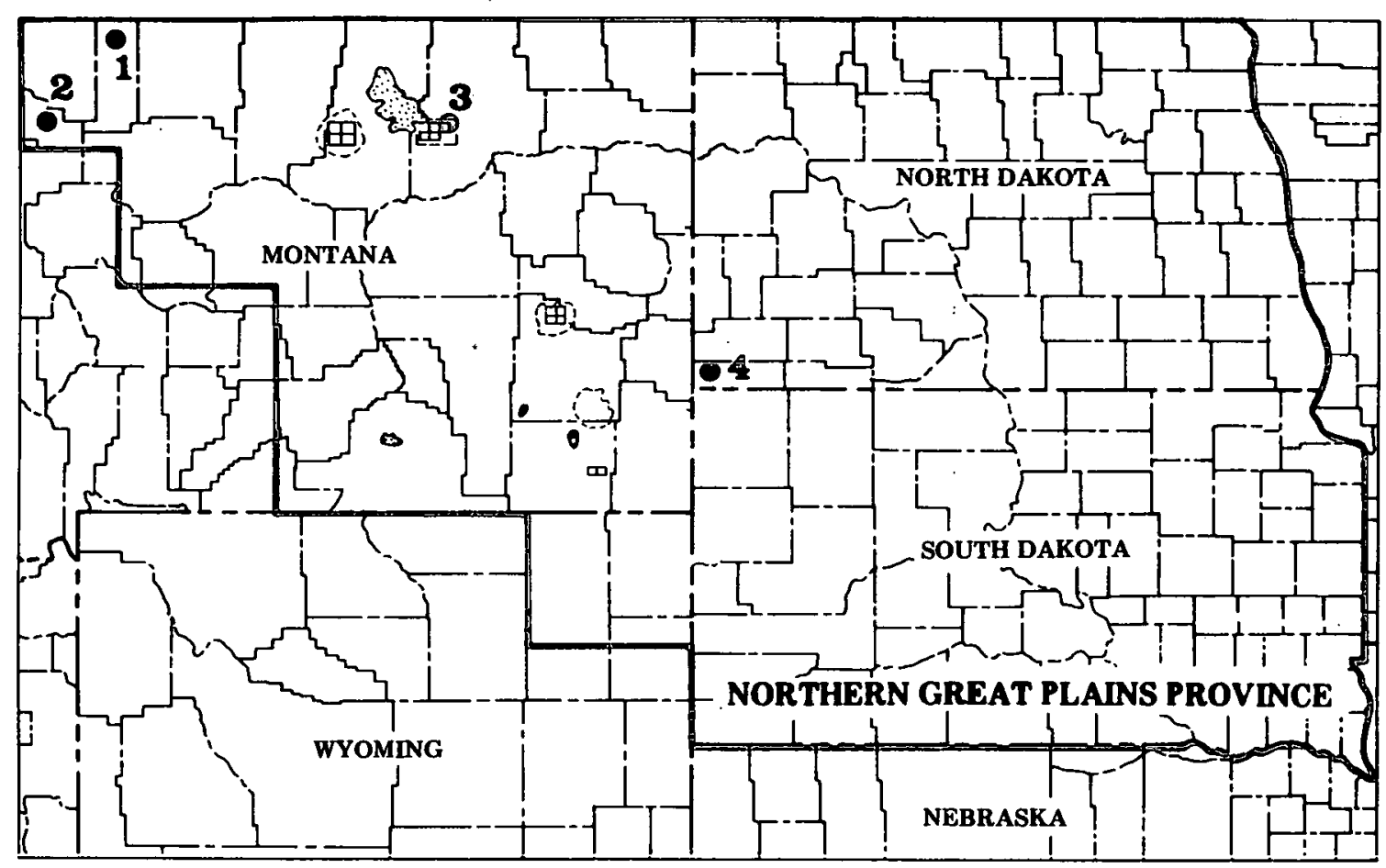

Figure 3-4 Northern Great Plains Province Showing Wells of Interest and USGS Designated Core Areas (refer to Table 3-7)

Table 3-7 Summary of Wells - Northern Great Plains Province

\begin{tabular}{|c|c|c|c|c|c|c|c|c|}
\hline OPERATOR & $\begin{array}{l}\text { WELL } \\
\text { NAME }\end{array}$ & $\begin{array}{c}\text { MAP } \\
\text { WDEX } \\
\text { NO.' }\end{array}$ & $\begin{array}{l}\text { LOCATION } \\
\text { Sec/T/R }\end{array}$ & $\underset{\text { ft }}{\text { HORIzON }}$ & $\begin{array}{c}\text { MINAL } \\
\text { TD }\end{array}$ & $\begin{array}{l}\text { FRACTURE } \\
\text { TREATMENT }\end{array}$ & STATUS & $\begin{array}{l}\text { IPF in } \\
\text { MCED }\end{array}$ \\
\hline Petroquest & $\begin{array}{l}1 \text { Gilson } \\
\text { D Riecke }\end{array}$ & 1 & $\begin{array}{l}\text { nwne } 17 / 37 N / 6 E \\
\text { Sage Creek Field } \\
\text { Lberty Caty, MT }\end{array}$ & $\begin{array}{l}\text { Blackleaf } \\
(1,801-1,834) \\
\text { Bow Island } \\
(2,238-2,244)\end{array}$ & 2,276 & $\begin{array}{l}\text { Acidized } \\
\text { with } 250 \\
\text { gal. Fract } \\
\text { w/1,000 } \\
\text { gal water } \\
5,000 \text { lb } \\
\text { sand. }\end{array}$ & $\begin{array}{l}\text { Develop. well } \\
\text { comp. 11-20-79 } \\
\text { as SIGW. Com- } \\
\text { mingled. }\end{array}$ & $\mathbf{N} / \mathbf{A}$ \\
\hline
\end{tabular}

$\begin{array}{lllll}\begin{array}{l}\text { Balcron } \\ \text { Oil }\end{array} & \begin{array}{l}6-31 \\ \text { Iverson }\end{array} & 2 & \begin{array}{l}\text { nwnw 31/29N/1W } \\ \text { Ledger Field }\end{array} & \begin{array}{l}\text { Bow Island } \\ \text { (879-840) } \\ \text { (open hole) }\end{array}\end{array}$

840

Wildcat well

comp. 8-18-79.

400

Bow Island

Extension-

Ledger Field.

No cores or

tests.

\begin{tabular}{|c|c|c|c|c|}
\hline $\begin{array}{l}\text { J. J. C. } \\
\text { Paine \& } \\
\text { Associates }\end{array}$ & $\begin{array}{l}1-0407 \\
\text { Strommen }\end{array}$ & 3 & $\begin{array}{l}\text { nesw } 4 / 30 \mathrm{~N} / 37 \mathrm{E} \\
\text { Unnamed Field } \\
\text { Valley Cnty, MT }\end{array}$ & $\begin{array}{l}\text { Greenhom } \\
(1,375)\end{array}$ \\
\hline $\begin{array}{l}\text { J. J. C. } \\
\text { Paine \& } \\
\text { Associates }\end{array}$ & 1 Carpenter & 4 & $\begin{array}{l}\text { n1/2sw } 33 / 130 N / \\
106 w \\
\text { Little Missouri } \\
\text { Field } \\
\text { Bowman Cnty, ND }\end{array}$ & $\begin{array}{l}\text { Eagle } \\
(1,166 \\
1,298)\end{array}$ \\
\hline
\end{tabular}

1,425

Wildcat well suspended, wOCT. Located in Core Area $\mathrm{C}$.

$1,444 \quad 18,026 \mathrm{gal}$ water $50,000 \mathrm{lb}$ sand Develop. well comp. 8-2-79.

No cores or tests. 
Figure 3-5

Piceance Basin Showing Wells of Interest and USGS Designated Core Areas (refer to Table 3-8)

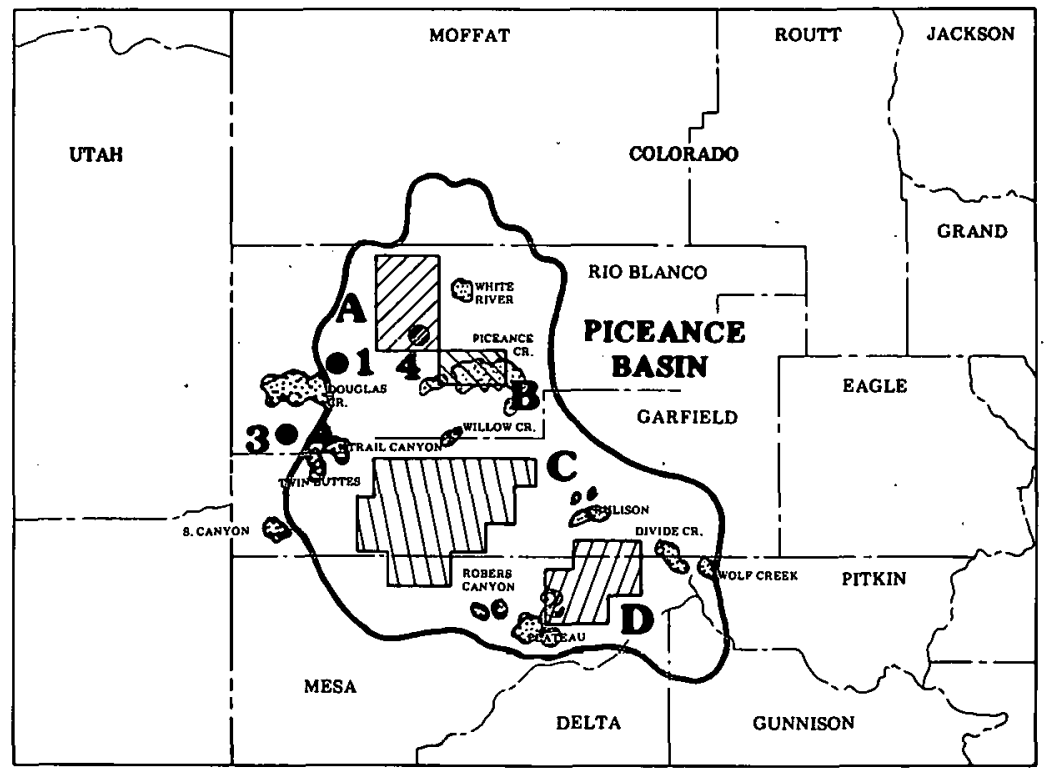

Table 3-8 Summary of Wells - Piceance Basin

\begin{tabular}{|c|c|c|c|c|c|c|c|c|}
\hline OPERATOR & $\begin{array}{l}\text { WELL } \\
\text { NAME }\end{array}$ & $\begin{array}{c}\text { MAP } \\
\text { NDEX } \\
\text { NO.! }\end{array}$ & $\begin{array}{l}\text { LOCATION } \\
\text { Sec/T/R }\end{array}$ & $\underset{\text { ft }}{\text { HORIZON }}$ & $\begin{array}{l}\text { FINAL. } \\
\text { TD }\end{array}$ & $\begin{array}{l}\text { FRACTURE } \\
\text { TREATMENT }\end{array}$ & STATUS & $\begin{array}{l}\text { IPF in } \\
\text { MCFD }\end{array}$ \\
\hline $\begin{array}{l}\text { Northwest } \\
\text { Exploration }\end{array}$ & $\begin{array}{l}16 \text { Phila- } \\
\text { delphia } \\
\text { Creek }\end{array}$ & 1 & $\begin{array}{l}\text { nwse } 11 / 2 S / 101 W \\
\text { Cathedral Fleld } \\
\text { Rio Blanco Cnty. } \\
\text { CO }\end{array}$ & $\begin{array}{l}\text { Mancos B } \\
(\mathbf{3 , 0 2 0}- \\
\mathbf{3 , 2 9 6 )}\end{array}$ & $\begin{array}{l}3.531 \\
\text { PB: } \\
3.460\end{array}$ & $\begin{array}{l}\text { Acidized w/ } \\
1,000 \text { gal. } \\
\text { Fract. w/ } \\
50,700 \text { gal } \\
\text { emul, } \\
50,000 \text { lb } \\
\text { sand. }\end{array}$ & $\begin{array}{l}\text { Develop. well } \\
\text { comp. 11-9-78. } \\
\text { Operator has } 4 \\
\text { other wells } \\
\text { producing in } \\
\text { the area. }\end{array}$ & 746 \\
\hline $\begin{array}{l}\text { Adolph } \\
\text { Coors }\end{array}$ & $\begin{array}{l}\text { CO 1-3 } \\
\text { Acco-. } \\
\text { Long }\end{array}$ & 2 & $\begin{array}{l}\text { nwnw } 3 / 10 S / 95 W \\
\text { Plateau Field } \\
\text { Mesa Cnty, Co }\end{array}$ & $\begin{array}{l}\text { Rollins } \\
(4,467-4,511) \\
\text { Cozzette } \\
(4,812-4,850) \\
\text { Corcoran } \\
(4,961-5,051)\end{array}$ & 5,120 & $\begin{array}{l}252,000 \mathrm{gal} \\
\text { water } \\
422,000 \mathrm{lb} \\
\text { sand }\end{array}$ & $\begin{array}{l}\text { Develop. well } \\
\text { comp. 5-25-78. } \\
\text { Commingled. } \\
\text { No cores or tests. }\end{array}$ & 1,087 \\
\hline $\begin{array}{l}\text { Coseka } \\
\text { Resources }\end{array}$ & $\begin{array}{l}10-21-4 \\
102 \\
\text { Kirby- } \\
\text { Robertson }\end{array}$ & 3 & $\begin{array}{l}\text { swne } 21 / 4 \mathrm{~S} / 102 W \\
\text { Thunder Field } \\
\text { Rio Blanco Cnty, } \\
\text { CO }\end{array}$ & $\begin{array}{l}\text { Mancos B } \\
(5,614-5,652) \\
\text { Dakota } \\
(5,652-5,795)\end{array}$ & $\mathbf{5 , 7 9 5}$ & & $\begin{array}{l}\text { Develop, well } \\
\text { comp. 6-30-79, } \\
\text { open hole com- } \\
\text { mingled. No } \\
\text { cores or tests. }\end{array}$ & 2,428 \\
\hline $\begin{array}{l}\text { Teton } \\
\text { Energy }\end{array}$ & $\begin{array}{l}\text { 8-2 } \\
\text { Yellow } \\
\text { Creek- } \\
\text { Federal }\end{array}$ & 4 & $\begin{array}{l}\text { nwnw } 8 / 1 \$ / 98 W \\
\text { Wildcat Fleld } \\
\text { Rio Blanco Cnty. } \\
\text { CO }\end{array}$ & $\begin{array}{l}\text { Mesaverde } \\
(8,500)\end{array}$ & & & $\begin{array}{l}\text { Wildcat well } \\
\text { reported } 8-31-79 \text {, } \\
\text { located in Core } \\
\text { Area } A \text {. }\end{array}$ & \\
\hline
\end{tabular}

1 Refer to Figure 3-5

Horizon - projected depth

or producing interval 


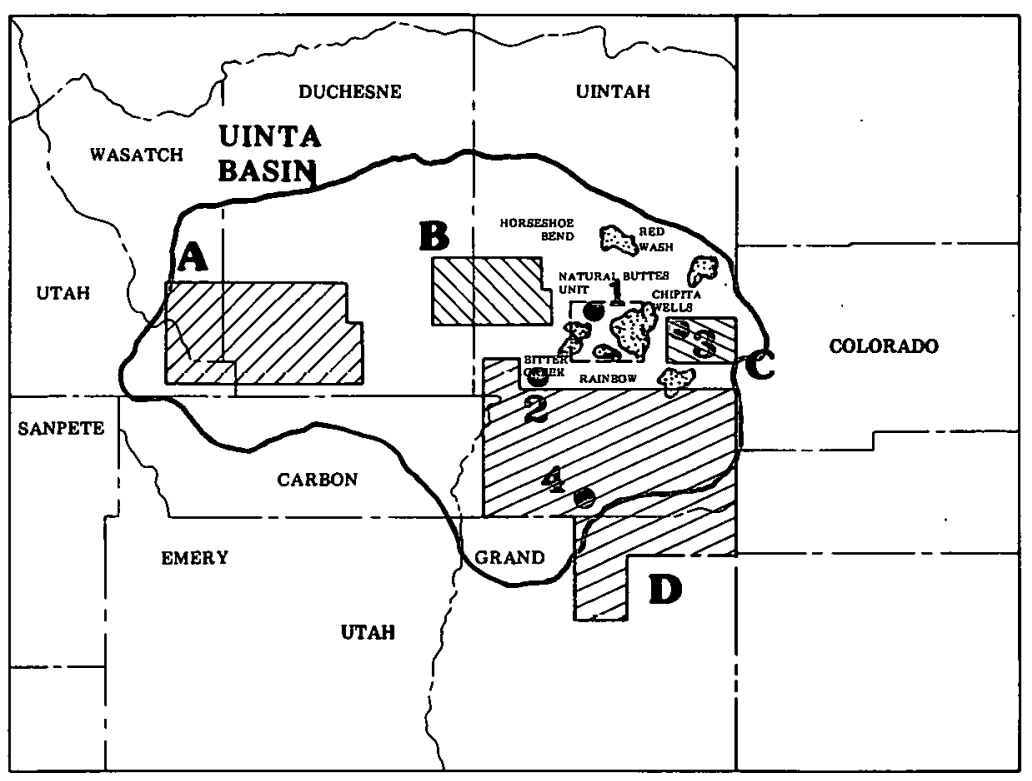

Figure 3-6

Uinta Basin Showing

Wells of Interest and USGS Designated Core Areas (refer to Table 3-9)

Table 3-9 Summary of Wells - Uinta Basin

\begin{tabular}{|c|c|c|c|c|c|c|c|c|}
\hline OPERATOR & $\begin{array}{l}\text { WELL } \\
\text { NAME }\end{array}$ & $\begin{array}{c}\text { MAP } \\
\text { NDEX } \\
\text { NO. }\end{array}$ & $\begin{array}{l}\text { LOCATION } \\
\text { Sec/T/R }\end{array}$ & $\underset{f t}{\text { HORIZON }}{ }^{2}$ & $\begin{array}{c}\text { FUNAL } \\
\text { TD }\end{array}$ & $\begin{array}{l}\text { FRACTURE } \\
\text { TREATMENT }\end{array}$ & STATUS & $\begin{array}{l}\text { IPF in } \\
\text { MCFD }\end{array}$ \\
\hline $\begin{array}{l}\text { Continental } \\
\text { Oll }\end{array}$ & $\begin{array}{l}35-5 \\
\text { Conoco- } \\
\text { Federal }\end{array}$ & 1 & $\begin{array}{l}\text { nwnw } 35 / 85 / 21 E \\
\text { Natural Buttes } \\
\text { Field } \\
\text { Uintah Cnty, UT }\end{array}$ & $\begin{array}{l}\text { Wasatch } \\
(5,911-6,483)\end{array}$ & $\begin{array}{l}7,568 \\
\text { PB: } \\
6,700\end{array}$ & $\begin{array}{l}\text { Acidized } \\
\text { and Fract. }\end{array}$ & $\begin{array}{l}\text { Develop. well } \\
\text { comp. 8-13-79. } \\
\text { No cores or tests. }\end{array}$ & 1,250 \\
\hline Wexpro & $\begin{array}{l}9 \text { Island } \\
\text { Unit }\end{array}$ & 2 & $\begin{array}{l}\text { nwsw } 11 / 10 S / 19 E \\
\text { Lsland Field } \\
\text { Uintah Cnty. UT }\end{array}$ & $\begin{array}{l}\text { Wasatch } \\
(6,088-6,151)\end{array}$ & $\begin{array}{l}6,800 \\
\text { PB: } \\
6.730\end{array}$ & $\begin{array}{l}\text { Acidized w/ } \\
3,000 \text { gal. } \\
\text { Fract. w/ } \\
76,000 \text { gal } \\
\text { gel, } 186,000 \\
\text { lb sand }\end{array}$ & $\begin{array}{l}\text { Develop. well } \\
\text { comp. 8-6-79. } \\
\text { No cores or tests. }\end{array}$ & 5,247 \\
\hline $\begin{array}{l}\text { Pacific } \\
\text { Transmission } \\
\text { Supply }\end{array}$ & $\begin{array}{l}\text { 23-17 } \\
\text { Federal }\end{array}$ & 3 & $\begin{array}{l}\text { nesw } 17 / 8 S / 23 \mathrm{E} \\
\text { Wild cat Field } \\
\text { Uintah Cnty. UT }\end{array}$ & $\begin{array}{l}\text { Mesaverde } \\
(8,800)\end{array}$ & $\cdot$ & & $\begin{array}{l}\text { Wildcat well } \\
\text { reported 9-24-79. } \\
\text { Located in Core } \\
\text { Area C. }\end{array}$ & \\
\hline $\begin{array}{l}\text { Coseka } \\
\text { Resources }\end{array}$ & $\begin{array}{l}\text { 3-11-1 5-21 } \\
\text { Wolf-Unit } \\
\text { Federal }\end{array}$ & 4 & $\begin{array}{l}\text { sesw } 11 / 15 \mathrm{~S} / 21 \mathrm{E} \\
\text { Wildcat Field } \\
\text { Uintah Cnty. UT }\end{array}$ & $\begin{array}{l}\text { Morrison } \\
(10,425)\end{array}$ & & & $\begin{array}{l}\text { Wildcat well } \\
\text { reported 9-24-79. } \\
\text { Located in Core } \\
\text { Area D. }\end{array}$ & \\
\hline
\end{tabular}

\footnotetext{
${ }^{1}$ Refer to Figure 3.6

2 Horizon - projected depth

or producing interval
} 
- the northern Rock Springs Uplift, located on the southeastern edge of the Green River Basin.

The formation nomenclature is somewhat complex in the three chosen areas. The northern area Paleocene deposits are called the Hoback formation. The upper Cretaceous nonmarine sequence is called the Mesaverde and is locally divided, from top to bottom, into the Harebell, White sandstone, and Lenticular sequence.

The Paleocene in the western area is called the Upper Evanston, Paleocene or Tertiary-1. The Mesaverde equivalents in this area are the Lower Evanston and Adaville formations, and are separated by a local unconformity.

The southeastern Green River Basin nomenclature is the most widely used. The Paleocene deposits are called the Ft. Union formation. This is underlain by the Cretaceous nonmarine Lance formation, which is separated from the Mesaverde by the marine Fox Hills sandstone and the Lewis Shale. The non-marine Mesaverde units are, from top to bottom, the Lower Almond, the Ericson, and Upper and Middle Rock Springs.

Preliminary work to get representative Paleocene and non-marine upper Cretaceous coverage in the three areas indicates better sandstone continuity than previous studies encountered in both the Piceance and Uinta Basins. However, discordant small scale sedimentary structures are more prevalent, especially in the northern Rock Springs Uplift area, and may be an important factor in low permeabilities reported in the subsurface.

The Green River Basin field work is projected to be completed in October.

\subsubsection{Reservoir Characteristics in Uinta Basin Gas Wells}

Log analysis to determine porosity, water saturations, and net pay thickness has been completed for 8 Uinta gas wells. Also, Homer plots have been developed to determine permeability and average reservoir pressures.

The results of these analyses and the production volume data for the wells will be used to check reservoir geometries established by studies of outcrops on the edge of the basin.

In order to verify late-time slopes on the Hormer plots, additional pressure measurements will be made on most of these wells prior to being put back on production. Mountain Fuel Supply Company is scheduled to place the wells on production by November 1,1979 . 
THIS PAGE

\section{WAS INTENTIONALLY LEFT BLANK}




\section{RESEARCH AND DEVELOPMENT BY ENERGY TECHNOLOGY CENTERS AND NATIONAL LABORATORIES}

\subsection{BARTLESVILLE ENERGY TECHNOLOGY CENTER}

\subsubsection{Improved Pressure Coring System - Sandia Laboratories}

\subsubsection{Core Retriever Design and Test}

Existing DOWDCO pressure core barrels have been modified and used in laboratory and field drilling tests of the high performance one- and two-piece core bits with PDC (Polycrystalline Diamond Compax) cutters and the Sandia low invasion coring fluid.

Laboratory drilling tests were conducted at Terra Tek's Drilling Research Laboratory in Salt Lake City. Both types of PDC bits and a standard natural diamond core bit were comparatively tested. When drilling in Berea Sandstone and Indiana Limestone, both with and without the Sandia low invasion fluid, the PDC bits outperformed the natural diamond core bit in penetration rate and core recovery. The two-piece PDC bit demonstrated a $50 \mathrm{ft}$ PRM (Penetration Rate per Minute) and the one-piece PDC bit's rates had as high as $120 \mathrm{ft}$. The standard natural diamond core bit gave only a $15.5 \mathrm{ft}$ PRM under similar conditions and cores obtained were of poorer quality.

Initial field tests of the single piece PDC core bit, using the modified pressure core barrels with the low invasion coring fluid and standard pressure core barrels without the low invasion fluid, have been conducted. Results are shown in Table 4-1.

Field testing will continue to evaluate the improved system's performance in other types of formations and in deviated holes, and to test additional design improvements as the hardware becomes available.

\subsubsection{Core Fluid Tests}

The patented magnesia additive was evaluated by adding 1 gram per liter of $\mathrm{Mg} 0$ to a base mud consisting of $10 \mathrm{lb} / \mathrm{BBL}$ HEC polymer and $50 \mathrm{lb} / \mathrm{BBL} \mathrm{CaCO}_{3}$ in 30 percent $\mathrm{CaCl}_{2}$ brine. The "stabilized" mud and the base mud were then tested for filtrate loss from $70^{\circ} \mathrm{F}$ to $300^{\circ} \mathrm{F}$, and the magnesia was found to significantly improve the fluid loss properties of the HEC. Similar tests performed with a proprietary polymer additive consisting of various starch derivatives and magnesia showed the starch to be of little value in reducing filtrate loss. The results are summarized on page 25 . 
Table 4-1 Field Test Results, Single Piece Stratapax@ Core Bit

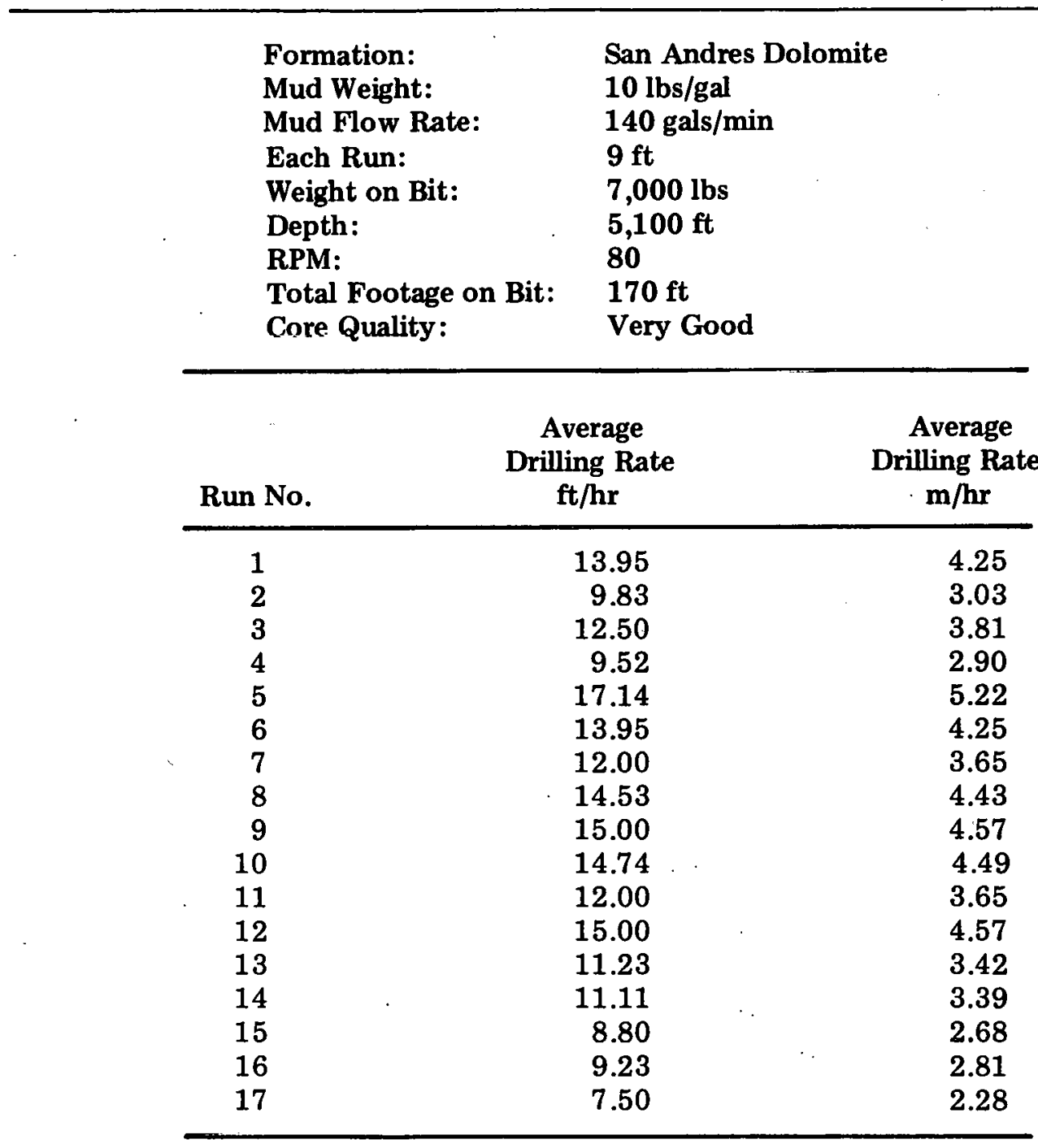

Standard natural diamond core bits used for coring in adjacent wells had penetration rates of 1 to $4 \mathrm{ft} / \mathrm{hr}$ with an average rate of $\approx 1.5 \mathrm{ft} / \mathrm{hr}$ and often produced much poorer quality. cores. 
Fluid Loss in 30 Minutes @ 500 psi $\Delta \mathrm{P}, \mathrm{cc}$

\begin{tabular}{lrcccr}
\hline & $70^{\circ} \mathrm{F}$ & $150^{\circ} \mathrm{F}$ & $200^{\circ} \mathrm{F}$ & $250^{\circ} \mathrm{F}$ & $300^{\circ} \mathrm{F}$ \\
Base Mud & 4.0 & 4.2 & 5.6 & 5.7 & $220 \pm$ \\
Mg0 & 2.9 & 4.0 & 5.0 & 4.3 & $10 \pm$ \\
Starch, Mg0 & 2.8 & 4.4 & 5.4 & 6.1 & $8 \pm$ \\
\hline
\end{tabular}

\section{4:1.1.3 Bit Design and Fabrication}

Redesign of the two-piece PDC core bit has been completed. Two main bit bodies and four pilot bit bodies are being fabricated. The pilot bit was redesigned to improve hydraulics with low invasion fluids and the main bit design was changed to accomodate the slimmed down pilot bits.

The changes were made to control the pilot bit core jamming problems as a result of the drilling tests at Terra Tek. Additional testing of the improved pressure coring system will be scheduled at the Drilling Research Laboratory as soon as hardware is available.

\subsubsection{Bit Tests}

Laboratory and field drilling tests of the PDC core bits demonstrated that the high penetration rates resulted in improved core quality.

A series of 9 test cores have been cut from the upper San Andres Dolomite in a Texas Pacific Oil Well near Denver City, Texas, using three designs of one-piece PDC bits with the low invasion fluid and a standard diamond core bit without the low invasion fluid: All cores were frozen in dry ice and shipped to Core Laboratories for analysis. Results will be reported when available.

\subsubsection{Interface Conductivity Effects on Electric Logging}

Modifications to the apparatus used to measure core resistance as a function of pressure and temperature are completed. Changes to the electrical measuring circuit were made to insure electrical isolation of the sample from current leakage paths.

\subsubsection{In Situ Permeability}

Measurement of Formation Characteristics of Western Tight Sands - Institute of Gas Technology

Work has commenced on the measurement of gas permeability of cores from the Twin Arrow C\&K No. 4-14 well in Rio Blanco County, Colorado, and from the Pacific Transmission Supply Company No. 24-19 Federal well located in Sublette County, Wyoming. The gas permeability of the core from the Twin Arrow well was found to be high (about $50 \mathrm{md}$ from a depth of $1,038.6 \mathrm{ft}$ ). The core samples are from shallow formations (maximum depth of cores studied was $1,144.5 \mathrm{ft}$ ). 
Table 4-2 Gas Permeability Measurements

Well: Twin Arrow, C\&K 4-14, Sec. 14 T3S R101W

Rio Blanco County, Colorado

\begin{tabular}{|c|c|c|c|c|c|}
\hline $\begin{array}{c}\text { Core } \\
\text { Depth } \\
\text { ft }\end{array}$ & $\begin{array}{c}\text { Net Confining } \\
\text { Pressure (PC) } \\
\text { psi }\end{array}$ & $\begin{array}{c}\text { Reciprocal } \\
\text { Mean Pressure, } \\
1 / \mathbf{P}_{\mathbf{M}} \times 10^{2}, \mathrm{psi}^{-1}\end{array}$ & $\begin{array}{l}\text { Permeability } \\
\text { md }\end{array}$ & $\begin{array}{l}\text { Klinkenberg } \\
\text { Permeability } \\
\text { Ko, md }\end{array}$ & $\begin{array}{l}\text { Permeability at a } \\
\text { Differential Pressure } \\
\text { of } 40 \mathrm{psi}, \mathrm{K}_{40} \text {, md }\end{array}$ \\
\hline \multirow[t]{3}{*}{995.5} & 120 & $\begin{array}{l}3.25 \\
2.83 \\
2.49\end{array}$ & $\begin{array}{l}2.43 \\
2.38 \\
2.33\end{array}$ & 2.01 & 2.38 \\
\hline & 500 & $\begin{array}{l}4.05 \\
3.34 \\
2.83\end{array}$ & $\begin{array}{l}2.24 \\
2.16 \\
2.08\end{array}$ & 1.72 & 2.09 \\
\hline & 1,000 & $\begin{array}{l}4.17 \\
3.29 \\
2.80\end{array}$ & $\begin{array}{l}2.02 \\
2.00 \\
1.99\end{array}$ & 1.93 & 1.99 \\
\hline \multirow[t]{3}{*}{$1,004.2$} & 200 & $\begin{array}{l}4.00 \\
3.26 \\
2.75\end{array}$ & $\begin{array}{l}5.77 \times 10^{-1} \\
5.14 \times 10^{-1} \\
4.87 \times 10^{-1}\end{array}$ & $3.32 \times 10^{-1}$ & $4.94 \times 10^{-1}$ \\
\hline & 500 & $\begin{array}{l}3.97 \\
3.28 \\
2.77\end{array}$ & $\begin{array}{l}4.78 \times 10^{-1} \\
4.50 \times 10^{-1} \\
4.31 \times 10^{-1}\end{array}$ & $3.22 \times 10^{-1}$ & $4.35 \times 10^{-1}$ \\
\hline & 1,000 & $\begin{array}{l}4.04 \\
3.31 \\
2.77\end{array}$ & $\begin{array}{l}3.82 \times 10^{-1} \\
3.53 \times 10^{-1} \\
3.33 \times 10^{-1}\end{array}$ & $2.26 \times 10^{-1}$ & $3.37 \times 10^{-1}$ \\
\hline
\end{tabular}

Gas permeability was measured as a function of mean pore pressure at three different confining pressures. Klinkenberg permeabilities $(K \infty)$ were estimated by extrapolating the measured data to a infinite pressure at each confining pressure. Examples of measured values and the Klinkenberg permeabilities of core from the Twin Arrow well are listed in Table 4-2.

Typical results of measurements made on core from the Pacific Transmission Supply Company well are listed in Table 4-3. The cores were obtained from depths ranging from 5,205 to $5,220 \mathrm{ft}$.

To facilitate comparison of permeability values, permeabilities at a mean pore pressure of 20 psig ( $\mathrm{K}_{40}$ ), corresponding to an upstream pressure of $40 \mathrm{psig}$ and a downstream pressure of 0 psig, are also listed in Tables 4-2 and 4-3. Core from the Twin Arrow well indicated a wide range of permeability, from 0.5 to $50 \mathrm{md}$; core from the Pacific Transmission Supply Company well exhibited a narrower range of permeability, from 0.15 to 0.52 md (at a net confining pressure of $120 \mathrm{psi}$ ). 
Table 4-3 Gas Permeability Measurements

Well: Pacific Transmission Supply Company No. 24-19 Federal Sec. 19 T33N R114W, Sublette County, Wyoming

\begin{tabular}{|c|c|c|c|c|c|}
\hline $\begin{array}{l}\text { Core } \\
\text { Depth } \\
\text { ft }\end{array}$ & $\begin{array}{c}\text { Net Confining } \\
\text { Pressure }\left(\mathbf{P}_{\mathbf{C}}\right) \\
\text { psi }\end{array}$ & $\begin{array}{l}\text { Reciprocal } \\
\text { Mean Pressure, } \\
\text { 1/P } \mathbf{P}_{\mathbf{M} \times 10^{2}, \mathrm{psi}}^{-1}\end{array}$ & $\begin{array}{c}\text { Permeability } \\
\text { md }\end{array}$ & $\begin{array}{l}\text { Klinkenberg } \\
\text { Permeability } \\
\mathbf{K} \infty \text {, md }\end{array}$ & $\begin{array}{l}\text { Permeability at a } \\
\text { Differential Pressure } \\
\text { of } 40 \text { psi, } K_{40}, \mathrm{md}\end{array}$ \\
\hline \multirow[t]{3}{*}{5,172} & 120 & $\begin{array}{l}4.04 \\
3.38 \\
2.86\end{array}$ & $\begin{array}{l}1.83 \times 10^{-1} \\
1.65 \times 10^{-1} \\
1.51 \times 10^{-1}\end{array}$ & $7.56 \times 10^{-2}$ & $1.52 \times 10^{-1}$ \\
\hline & 1,000 & $\begin{array}{l}4.07 \\
3.26 \\
2.85\end{array}$ & $\begin{array}{l}1.14 \times 10^{-1} \\
1.03 \times 10^{-1} \\
9.80 \times 10^{-2}\end{array}$ & $6.10 \times 10^{-2}$ & $9.80 \times 10^{-2}$ \\
\hline & 2,586 & $\begin{array}{l}4.04 \\
3.25 \\
2.82\end{array}$ & $\begin{array}{l}8.90 \times 10^{-2} \\
7.70 \times 10^{-2} \\
7.20 \times 10^{-2}\end{array}$ & $3.34 \times 10^{-2}$ & $7.26 \times 10^{-2}$ \\
\hline 5,220 & 120 & $\begin{array}{l}4.04 \\
3.33 \\
2.90\end{array}$ & $\begin{array}{l}5.85 \times 10^{-1} \\
5.37 \times 10^{-1} \\
5.20 \times 10^{-1}\end{array}$ & $3.49 \times 10^{-1}$ & $5.16 \times 10^{-1}$ \\
\hline$\circ$ & 1,000 & $\begin{array}{l}4.04 \\
3.30 \\
2.83\end{array}$ & $\begin{array}{l}4.31 \times 10^{-1} \\
4.00 \times 10^{-1} \\
3.67 \times 10^{-1}\end{array}$ & $2.24 \times 10^{-1}$ & $3.73 \times 10^{-1}$ \\
\hline & 2,610 & $\begin{array}{l}4.09 \\
3.39 \\
2.83\end{array}$ & $\begin{array}{l}3.34 \times 10^{-1} \\
2.96 \times 10^{-1} \\
2.76 \times 10^{-1}\end{array}$ & $1.43 \times 10^{-1}$ & $2.76 \times 10^{-1}$ \\
\hline
\end{tabular}

Permeability of Interbedding Material of Tight Western Gas Sands - Lawrence Livermore Laboratory

Construction and assembly of the permeability measurement system is proceeding and is approximately one-half completed. Debugging each of the sub-units will begin in October or November and the completed unit should be placed on line late in December. The computer program which will be used to analyze the data is being set up to include the effects of the compressibility of the gas.

\subsubsection{Advanced Logging Techniques and Interpretation}

Dielectric Constant Measurements for Formation Evaluation

Additional improvements have been made in the dielectric constant phase shift measurement system. The system measures dielectric constant in terms of a network phase shift. 
The system, which had been breadboarded, has been refined by using mixers to convert the radio frequency test signals to $20 \mathrm{kHz}$ signals for a phase shift measurement. An HP 5328A electronic counter, operating in the time interval averaging mode, was used to measure the phase shift accurately. Preliminary measurements show that a sensitivity of at least 5 counts per picofarad can be obtained with a $2 \mathrm{MHz}$ test frequency. Additional components have been ordered to improve the accuracy and frequency range of this system.

A second system for dielectric constant measurement will use digital sampling methods. The analog-to-digital converters and samplehold modules have been received. The control and readout circuits are being designed. This system should be more flexible than the present dielectric constant phase shift system.

A new dielectric constant sample holder has been designed which will-allow fluid saturation and dielectric constant measurement in the same unit. Construction is planned for October.

Instrumentation for Formation Evaluation and Advanced Logging Techniques - Sandia Laboratories

In the August WGSP status report, it was stated that the frequency-dependent electrical parameters of saturated sandstones could be represented by Archie's equation coupled with dielectric laws. It was further noted that a continuum of pore shapes would be required to describe the data of Poley, et al. Work has been done concerning the form of the required distribution functions.

One description of a relaxing dielectric is

$$
\epsilon(\omega)=\epsilon(\infty)+i \int_{0}^{\infty} \frac{A(k) d k}{\omega+i l}
$$

where $A(k)$ is the distribution function in frequency space. Several $A(k)$ functions were fit to the real part of $\epsilon(\omega)$ given by Poley, et al. Although some are complicated, a simple $A(k)$ represented by a histogram,

$$
\begin{aligned}
& A_{1}, 10^{7}<k<10^{8} \mathrm{~Hz}, \\
& A_{2}, 10^{5}<k<10^{7} \mathrm{~Hz},
\end{aligned}
$$

etc.

fits the data well. A test of the model $(\epsilon(\omega)$ agreeing with the data) was successful.

Work is underway to transform this frequency distribution function into distribution functions of preshapes.

\subsubsection{Nuclear Magnetic Resonance Studies - Los Alamos Scientific Laboratory}

Using a 9 in. electromagnet operated at $0.5 \mathrm{MHz}$, the remotely-produced region of homogeneous magnetic field was investigated to determine the sensitivity of NMR detection 
techniques with samples outside the coil. Several small receiver coils were made and the laboratory system tested. A coil (approximately $2.5 \mathrm{~cm} \mathrm{x} 4 \mathrm{~cm}$ long) con'structed to resonate at the logging frequency of approximately $0.5 \mathrm{MHz}$ was ușed successfully to detect signals from samples exterior to the coil of the laboratory electromagnet. An extensive set of measurements (using glycerol as the sample material) was made on the signal strength of various annular samples relative to a standard sample internal to the core.

\subsubsection{Reservoir Simulator Studies}

Parametric Analysis of MHF Test Data and Engineering Studies of Western Gas Sands Intercomp

The post-breakdown pressure buildup test data from zones $4 \mathrm{~A}$ and $4 \mathrm{~B}$ of the Mobil PCU F31-13G well have been analyzed by computer matching the test data. The analysis showed both zones to be fractured. The fractures may have been naturally occurring or created during the breakdown operation.

The analysis of the buildup test data from zone $4 \mathrm{~A}$ gives a fracture half-length, $\mathrm{x}_{\mathrm{f}}$, of $24 \mathrm{ft}$ and a fracture flow capacity, $\mathrm{k}_{\mathrm{f}} \mathrm{w}$, of $11.5 \mathrm{md}$-ft. The effective, $\mathrm{p}_{\mathrm{i}}$, was 4,140 psia.

The analysis of the pressure buildup data from zone 4B gives:

$$
\begin{aligned}
& \mathrm{x}_{\mathrm{f}}=72 \mathrm{ft} \\
& \mathrm{k}_{\mathrm{f}}=5.7 \mathrm{md}-\mathrm{ft} \\
& \mathrm{k}_{\mathrm{g}}=0.001 \mathrm{md} \\
& \mathrm{p}_{\mathrm{i}}=4,060 \mathrm{psia}
\end{aligned}
$$

A post-fracture pressure drawdown test on zone 9 was matched, varying only fracture properties. Reservoir properties determined by matching the pre-fracture pressure buildup data were held constant in matching the post-fracture drawdown pressures. The fracture and reservoir parameters obtained for post-fracture zone 9 were used to predict production rates for an extended flow test taken on the zone. The production rates predicted by the model match well with the rates measured during the test.

The analysis of the post-fracture pressure data from zone 9 gave a fracture half-length, $\mathrm{x}_{\mathrm{f}}$, of $1,367 \mathrm{ft}$ and a fracture flow capacity, $\mathrm{k}_{\mathrm{f}} \mathrm{w}$, of $190 \mathrm{md}-\mathrm{ft}$. The fracture face was damaged. A fracture skin factor of 0.065 was used in matching pressure from the postfracture drawdown test. Effective gas permeability in the reservoir was $0.021 \mathrm{md}$.

\subsubsection{Rock-Fluid Interaction}

\section{Gel Fracture Fluid Characterization}

Work continued on column preparation for better resolution of low molecular weight separation and resolution of samples with molecular weights greater than 2,000,000. Studies have continued on the filtration effects on light molecular weight gels prior to characterization in a liquid chromatographic system.

Mapping Water Resistivities in the Tight Western Gas Sands - Texas A\&M University 
A large packet of information generated in a previous investigation of water resistivity values in the Uinta Basin has been received. This includes eleven articles on the geology of the basin and surroundings, various maps, some graphical summaries of attempts to contour $R_{W}$ values, and a collection of several hundred analyses.

A cursory examination of a collection of test results from producing gas wells indicated at least a hundred fold variance in salinity. Histograms of the chloride content in each sample are being prepared to test the validity of the generalizations previously stated.

\section{Characterization of Sintered Bauxite Proppant}

A 4 in. diameter tight sandstone core from the Pacific Transmission Supply Company No. 24-19 Federal well $(5,171 \mathrm{ft})$ was cut into $13 / 16$ in. thick wafers and vacuum saturated with 2 percent $\mathrm{KCl}$ solution. Proppant embedment was tested between two of these wafers. Hardness of the core material varied randomly from 56 to 93 on the Rockwell B scale with an average of 80.9 . Size distribution of $12-20$ mesh sintered bauxite used is given in Table 4-4.

Table 4-4 Size Distribution of 12-20 Mesh Sintered Bauxite Proppant

\begin{tabular}{lccc}
\hline $\begin{array}{l}\text { Sieve } \\
\text { Size }\end{array}$ & $\begin{array}{c}\text { Size Range } \\
\text { in mm }\end{array}$ & $\begin{array}{c}\text { Weight } \\
\text { Percent }\end{array}$ & $\begin{array}{c}\text { Estimated Number } \\
\text { Percent }\end{array}$ \\
\hline $10-12$ & $2.000 \cdot 1.700$ & 2.9 & 0.6 \\
$12-14$ & $1.700-1.400$ & 23.9 & 8.3 \\
$14-16$ & $1.400 \cdot 1.180$ & 18.5 & 11.2 \\
$16-18$ & $1.180-1.000$ & 28.0 & 28.0 \\
$18-20$ & $1.000-0.850$ & 20.9 & 34.2 \\
$20-25$ & $0.850-0.710$ & 4.7 & 12.8 \\
$25-30$ & $0.710-0.600$ & 0.9 & 4.0 \\
$30-35$ & $0.600-0.500$ & 0.2 & 1.2 \\
$35-40$ & $0.500-0.425$ & $<0.1$ & $<0.1$ \\
\hline
\end{tabular}

Fifty, 75 and 100 percent of a monolayer of proppant were used, with a complete monolayer being $1.72 \pm 0.05 \mathrm{~g} / \mathrm{in}^{2}$. The total proppant area was $3.98 \mathrm{in} .2$ (a $2-1 / 4$ in. diameter circle). In order to facilitate proppant placement, one member of each core section pair was coated with silicone grease (Dow-Corning High Vacuum Silicone Lubricant). Prior to an embedment test, the proppant-covered core section was soaked in the 2 percent $\mathrm{KCl}$ brine, thereby wetting the sintered bauxite. The assembly used for the embedment measurements is shown in Figure 4-1.

The decrease in distance $(\Delta \mathrm{l})$ from the comers of the steel plate to the base of the press with applied pressure was measured with a spring loaded dial micrometer. Steel stops were placed on the comers of the $1 / 8$ in. steel plate to increase reproducibility when moving the 


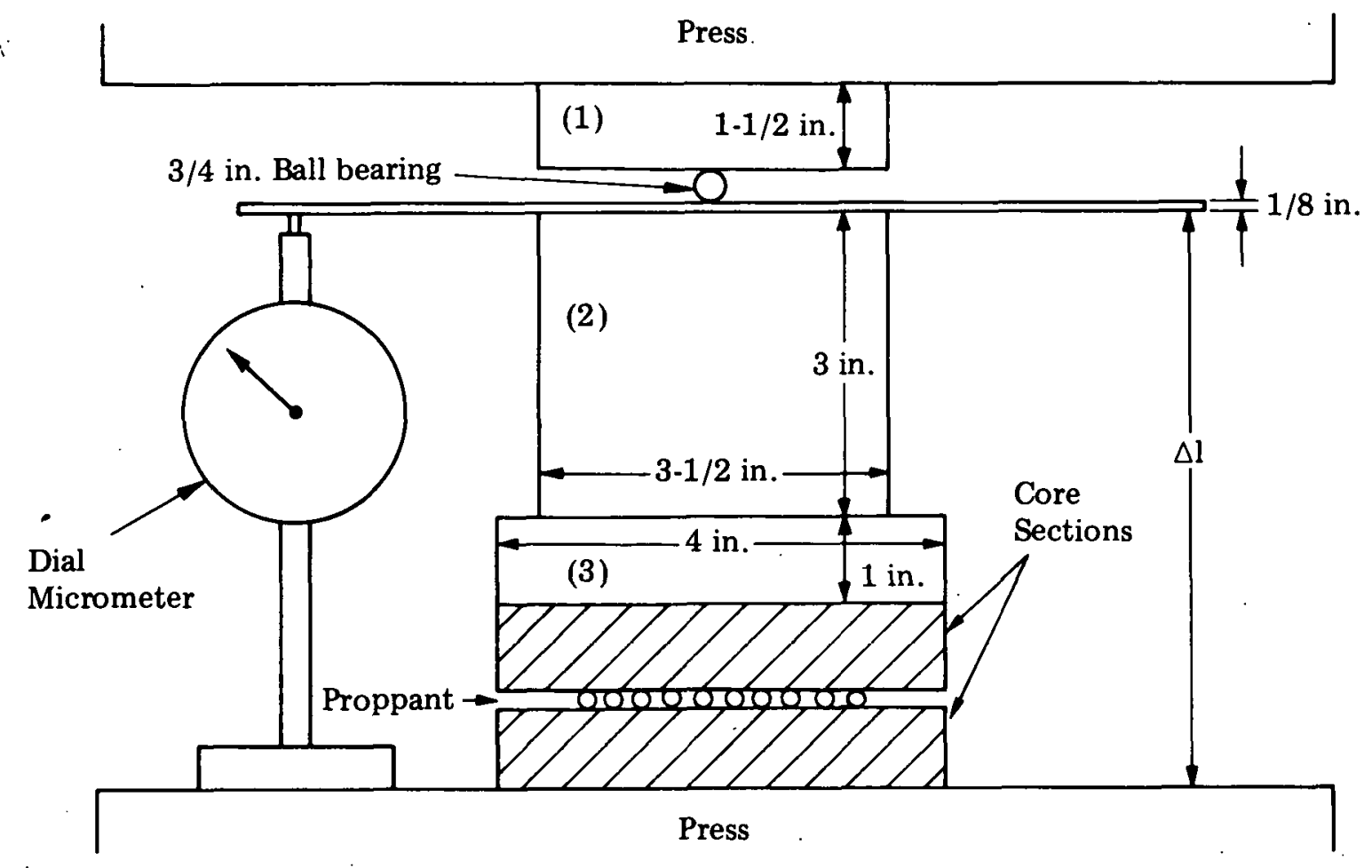

Figure 4-1 Proppant Embedment Apparatus. (1), (2) and (3) are of Hardened Steel. The 1/8 in. Steel Plate has a 1 in. Hole Cut in its Center to Allow Clearance for the Ball Bearing. This Steel Plate is Epoxied onto Steel Cylinder (2).

dial micrometer. This reproducibility was found to be +0.0002 in. At each pressure, the $\Delta \mathrm{ls}$ for each corner were averaged to give a single representative value, $\Delta \mathrm{l}^{\prime}$.

The compression of the hardened steel cylinder, (2), and steel plate, (3), in Figure 4-1 was found to be only 1-2 percent of the embedment change. The compressibility of the core itself was measured, but due to the irregular point-like support offered by the proppant, the actual compression of the core sections during an experiment was not known. However, corrections for the core compression (somewhat overestimated) and for the compression of the steel members (2) and (3) were attempted. Embedment results are presented in Figures 4-2, 4-3 and 4-4 for 100 percent, 75 percent, and 50 percent monolayer proppant coverage, respectively. In these figures, the percent closure was based on a $1.90 \mathrm{~mm}(0.0739 \mathrm{in}$. fracture width, which was the diameter of the largest proppant found in the 12-20 mesh bauxite. The percent closure is the sum of the embedment of proppant into both faces. As in previous work, there was some proppant fracturing. In every experiment the core sections fractured between 1,000 and $4,000 \mathrm{psi}$. This was also evident in the $\Delta \mathrm{l}^{\prime}$ values obtained. The top graph in Figures 4-2, 4-3 and 4-4 is the proppant embedment (corrected for compression 


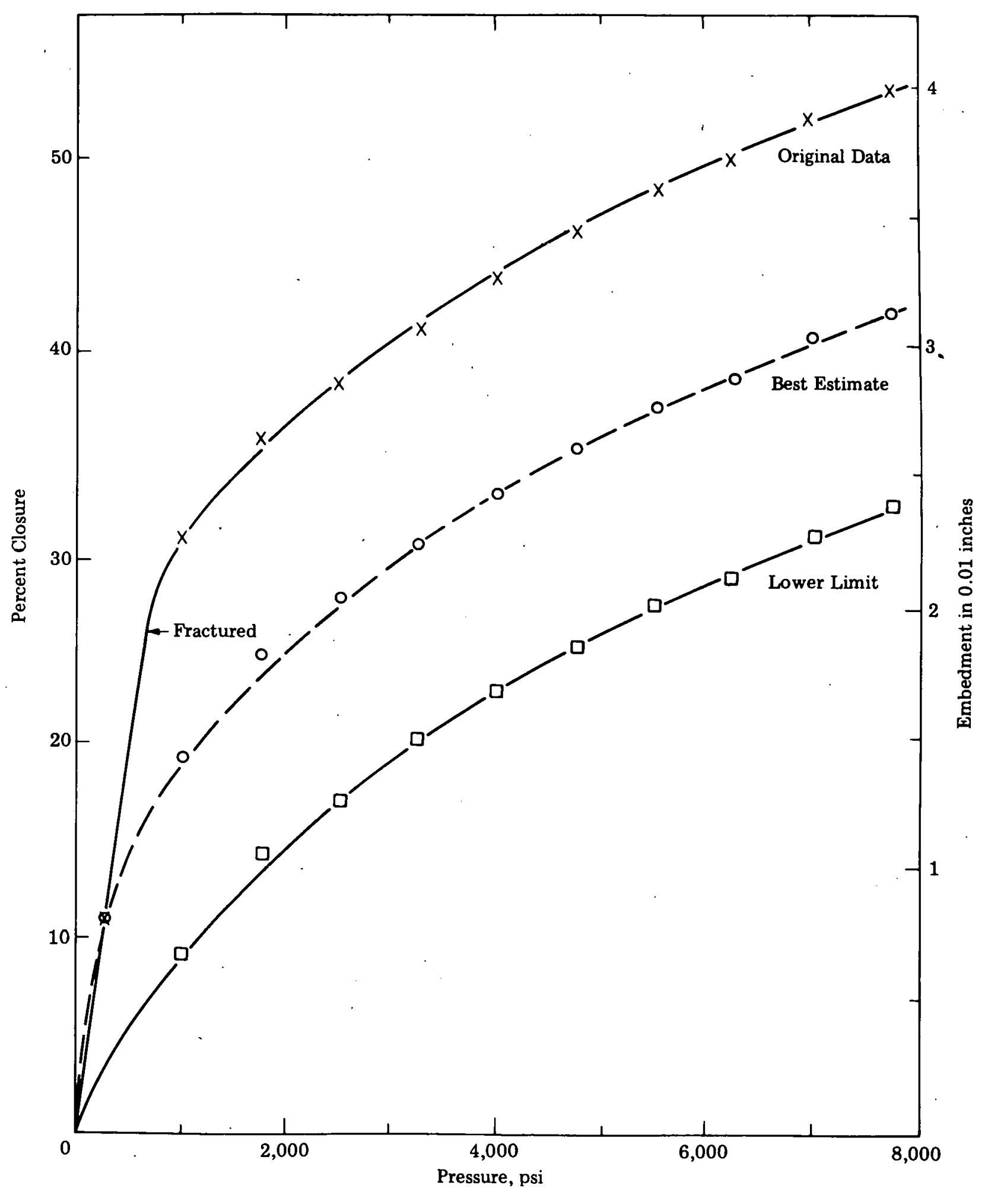

Figure 4-2 Percent Closure vs. Applied Pressure for 100 percent Coverage. 


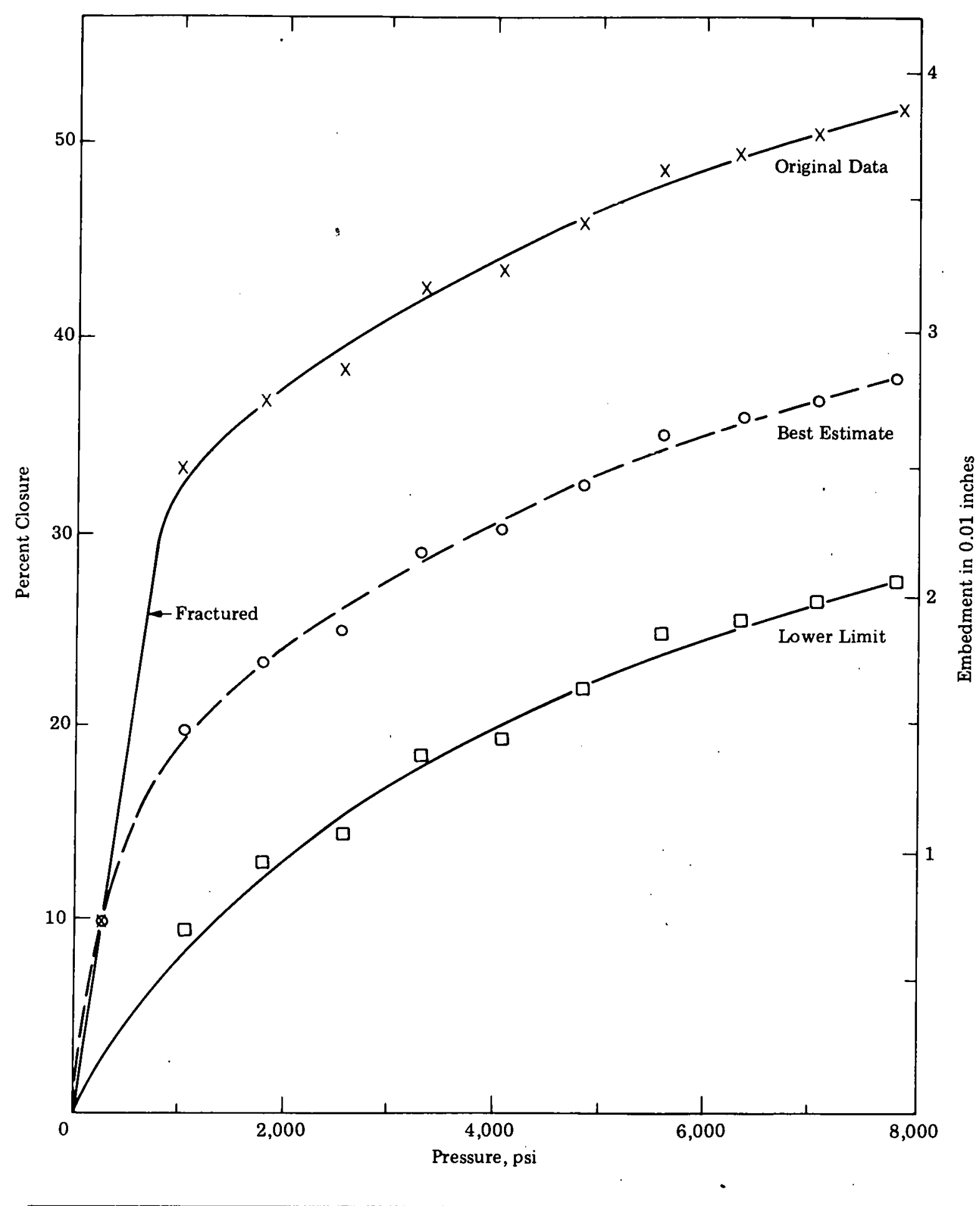

Figure 4-3 Percent Closure vs. Applied Pressure for 75 percent Coverage. 


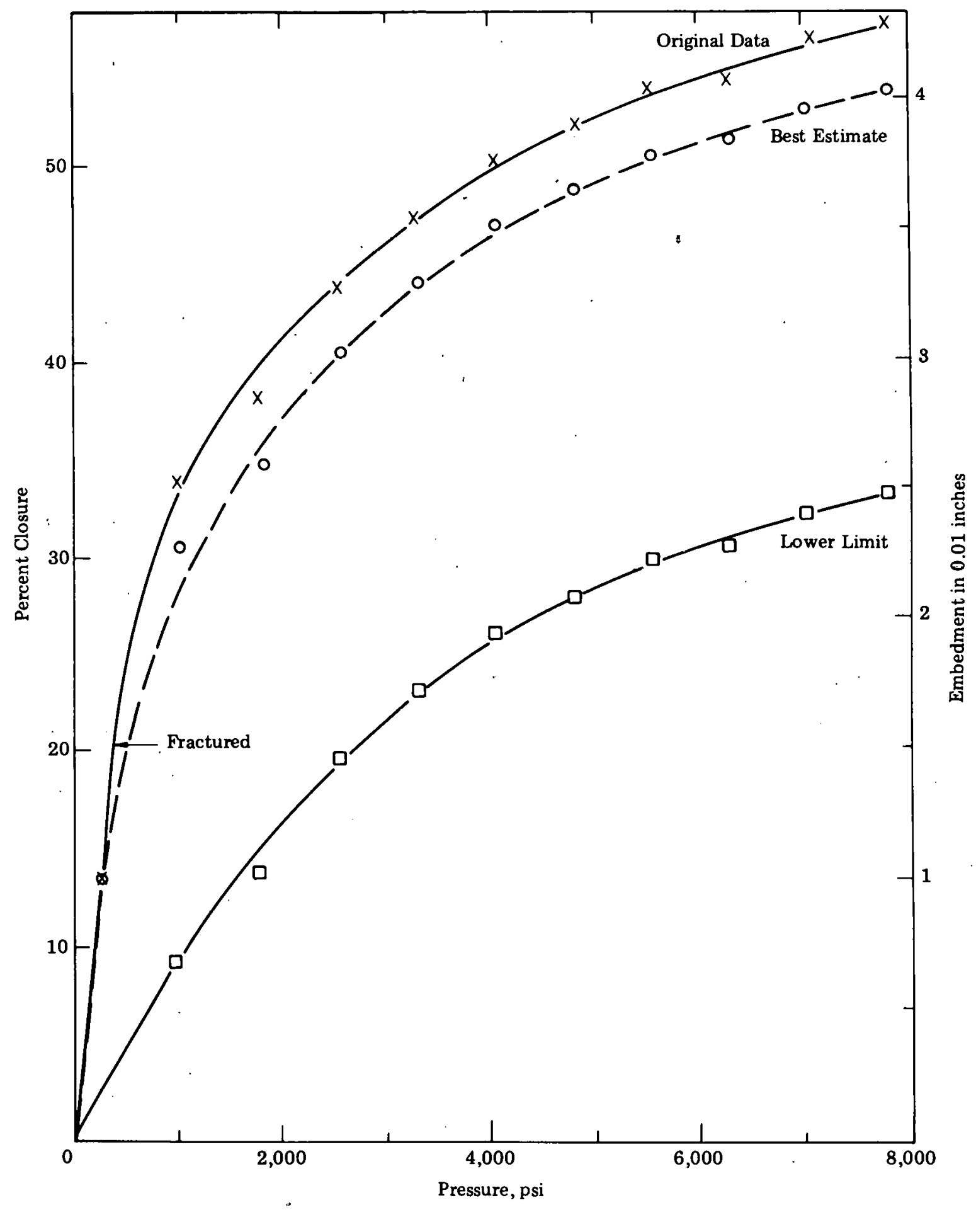

Figure 4-4 Percent Closure vs. Applied Pressure for 50 percent Coverage. 
of the core and steel components) without accounting for core fracturing. The lower graph was derived by drawing a smooth curve through the data at 4,000 psi and higher, followed by extrapolation back to the axis. This should represent a minimum proppant embedment, since past experience has shown that the change in $\Delta \mathrm{l}^{\prime}$ is considerably larger at lower applied pressures. The center curve (dashed) is a "best estimate" of the actual embedment. Here, the change in $\Delta \mathrm{l}^{\prime}$ between 1,000 and $4,000 \mathrm{psi}$ was varied until a reasonably smooth curve could be fit through all the data. This, in effect, involved vertically moving the data at 4,000 psi and above, attempting to compensate for the core fracturing. The error produced by this procedure is at least a couple of embedment percent at the higher pressures.

The opinion is that within the limits of this experiment the embedments at 100 percent and 75 percent proppant coverages are indistinguishable, and embedment at 50 percent coverage is somewhat greater. At $7,000 \mathrm{psi}$, a fracture propped with a 75 percent or greater monolayer with $12-20$ mesh bauxite will result in a 40 percent fracture closure based on a 1.90 $\mathrm{mm}$ fracture and a formation of the same general hardness. Reduction to 50 percent proppant coverage could increase the closure to 65 percent. At $2,500 \mathrm{psi}$, or at a depth of about 5,000 feet (the depth from which this core was taken), the closure would be 25 to 30 percent at proppant coverages of 75 percent or greater, and 40 percent with a 50 percent coverage. Therefore, although sintered bauxite may show some clear advantages over sand or glass beads, some consideration must be given to the problem of fracture closure through embedment.

Feasibility of Fabricating Light-weight Proppants for Application in Gas and Oil Well Stimulation - Terra Tek

During August, literature reviews were initiated to determine:

- patent and published works to determine previous work in selecting light weight proppants,

- properties of porous ceramics, and

- material properties data bank for selection of a composite proppant.

The literature survey for the first two items is complete. Initial review of the papers gathered indicated that for 30 to 40 percent porosity, material strength was reduced by 90 percent. The ceramics remained potentially viable light-weight proppants. The material properties data bank is expected to be completed by the end of October.

Analytical work is proceeding to analyze the stresses resulting from formation loading of a composite proppant.

\subsubsection{Schedule Status}

Figure $4-5$ is a milestone chart depicting BETC WGSP progress. 


\begin{tabular}{|c|c|c|c|c|c|c|c|c|c|c|c|c|c|}
\hline \multirow{2}{*}{\multicolumn{2}{|c|}{$\begin{array}{l}\text { WGSP-BETC } \\
\text { A. ROCK MECHANICS }\end{array}$}} & \multicolumn{12}{|c|}{ FY-79 } \\
\hline & & OCT & Nov & DEC & JAN & FEB & MAR & APR & MAY & JUN & JUL & AUG & SEP \\
\hline \multicolumn{14}{|c|}{$\begin{array}{l}\text { 1. FRACTURE \& ROCK } \\
\text { CHARACTERISTICS }\end{array}$} \\
\hline \multicolumn{14}{|c|}{ Design improved coring system } \\
\hline \multicolumn{14}{|c|}{ Core fluid testing } \\
\hline \multicolumn{14}{|c|}{ Coring tool report } \\
\hline \multicolumn{14}{|c|}{ Core Tests } \\
\hline Test N & Jo. 1 & $\%$ & $\nLeftarrow$ & $\infty \%$ & 20 & socs. & $\$ 2$ & $\$ 4$ & $40 \%$ & 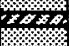 & $80 \%$ & $\$ \%$ & 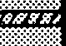 \\
\hline Test $N$ & No. 2 & & & 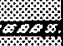 & & & $<$ & 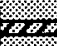 & $\infty$ & $1 \%$ & (2) & 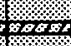 & $\$ 4 \times 4$ \\
\hline LEGEND & & & & & & & & & & & & & \\
\hline$\nabla$ & $\begin{array}{l}\text { Scheduled Start and Com- } \\
\text { pletion of Task }\end{array}$ & & & & & & & & & & & & \\
\hline$\underline{\nabla}$ & $\begin{array}{l}\text { Completed Milestone } \\
\text { Projected Schedule }\end{array}$ & & & & & & & & & & & & \\
\hline 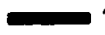 & Task Progressing & & & & & & & & & & & & \\
\hline 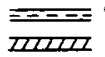 & $\begin{array}{l}\text { Task Progress Not Reported } \\
\text { Delay in Work on Task }\end{array}$ & & & & & & & & & & & & \\
\hline
\end{tabular}

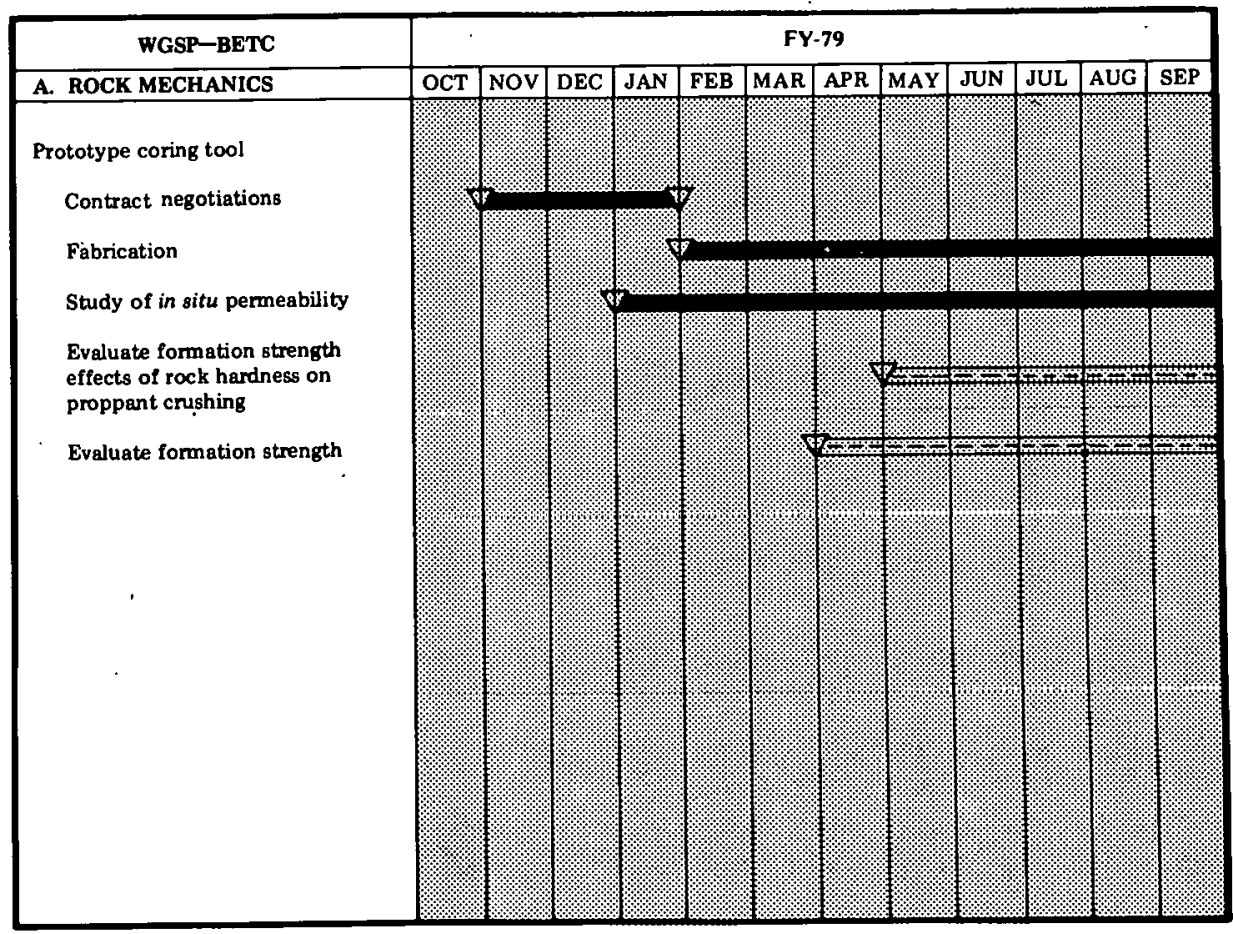

Figure 4-5 Milestone Chart - BETC 


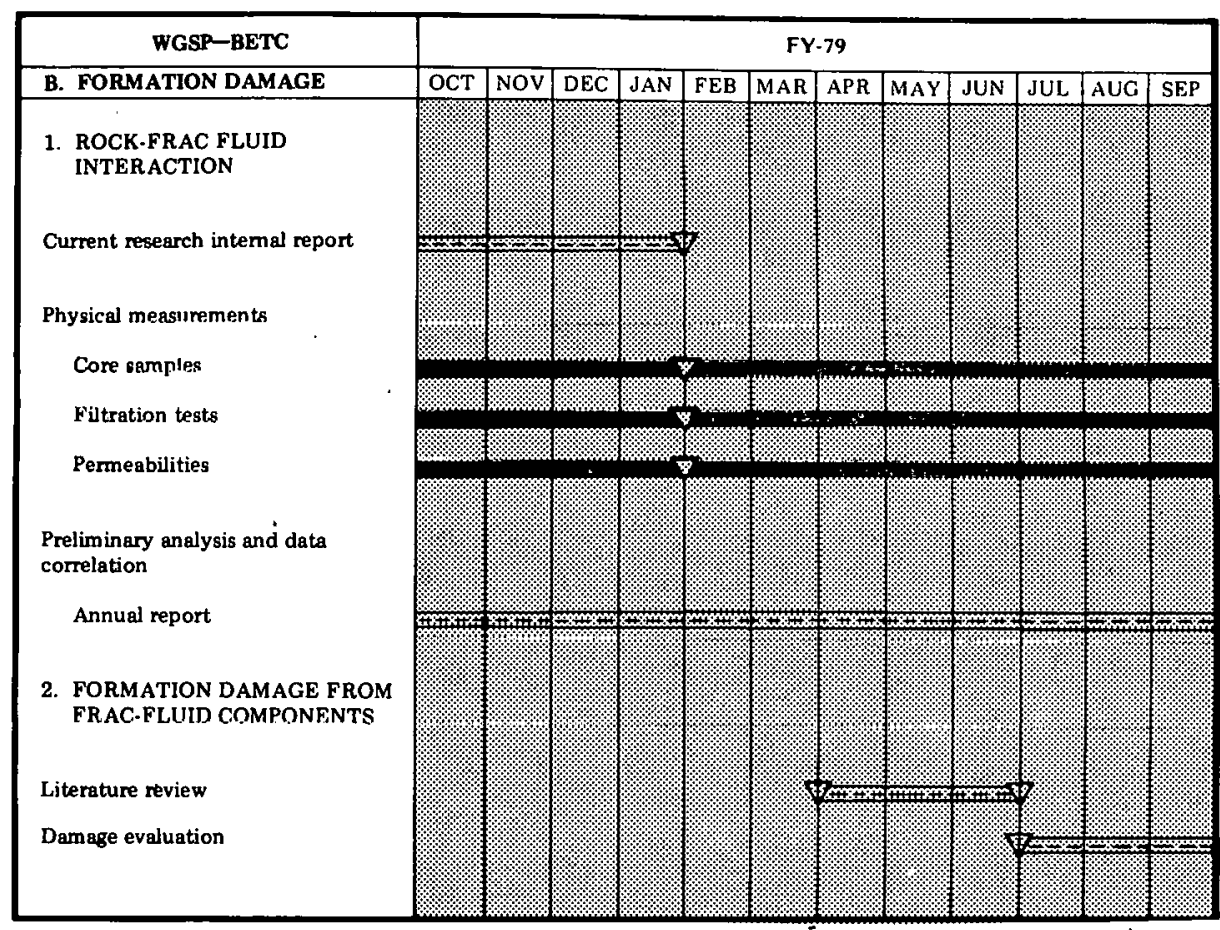

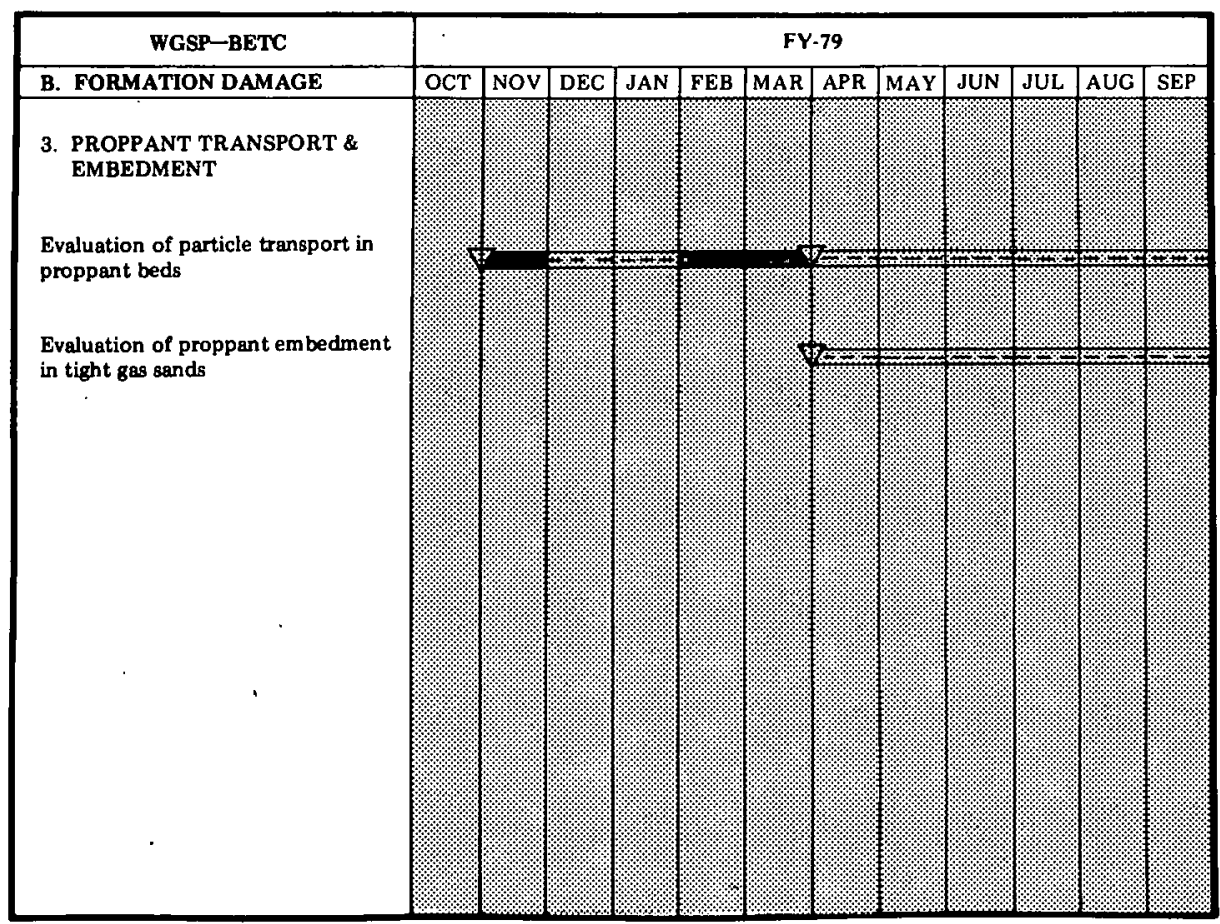

Figure 4-5 Continued 

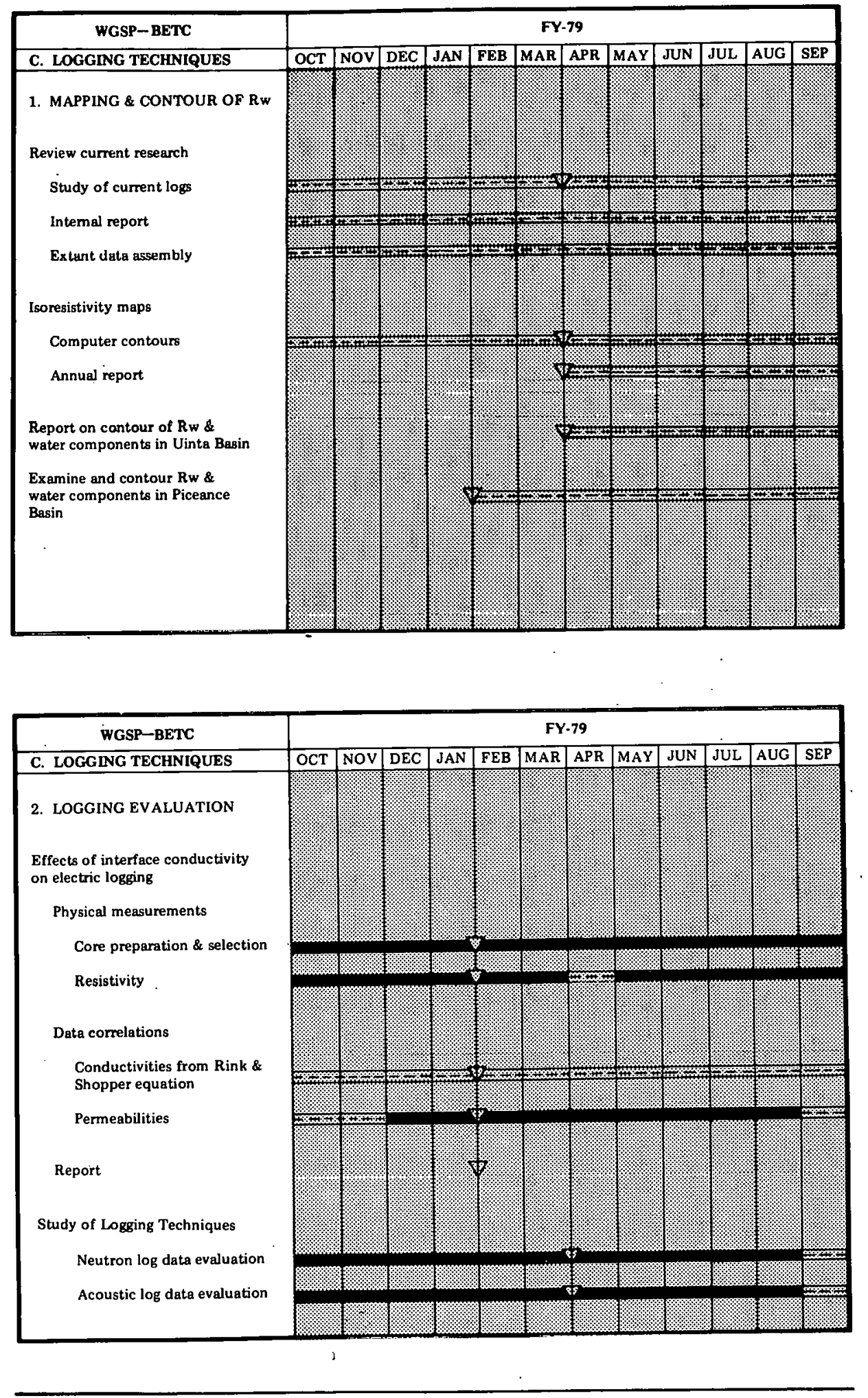

Figure 4-5 Continued 


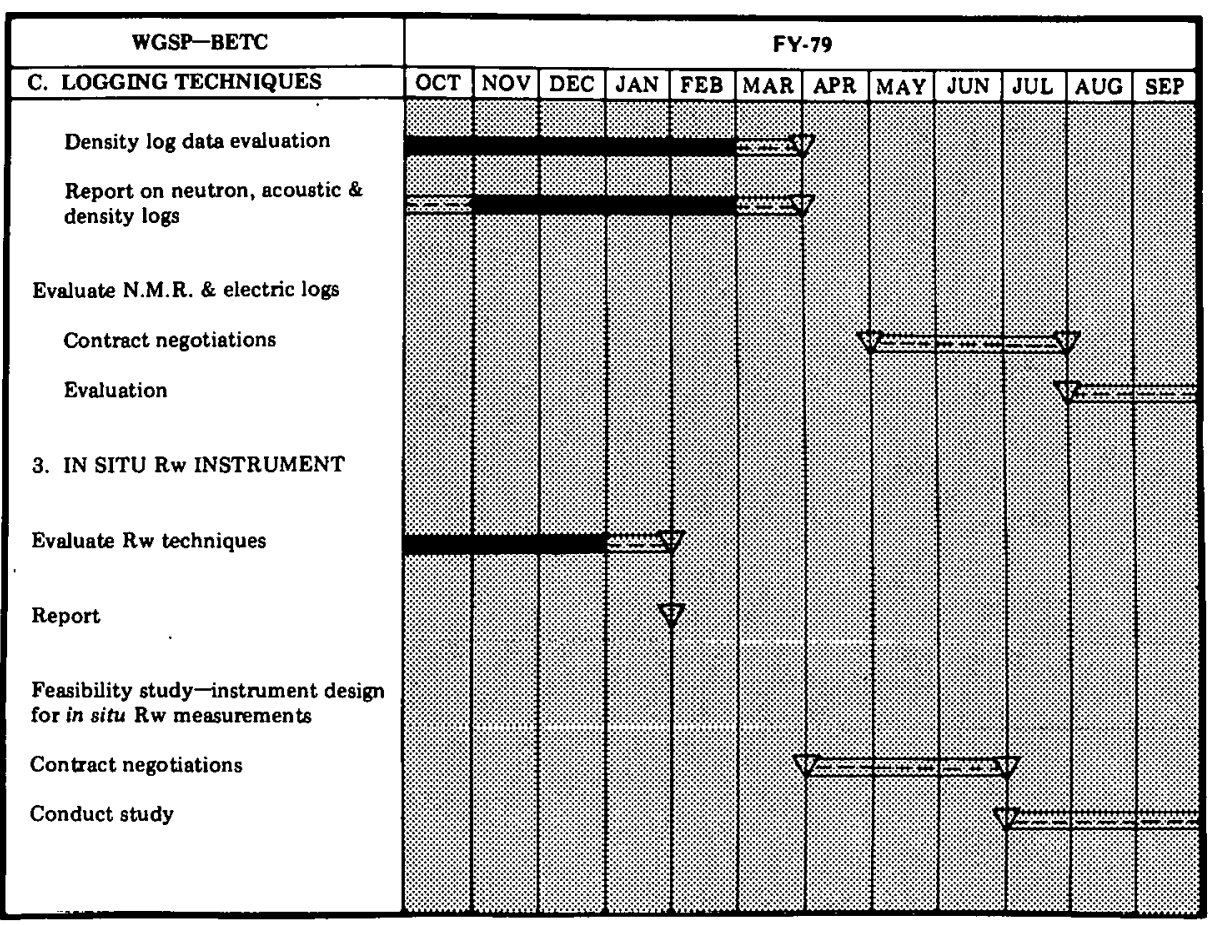

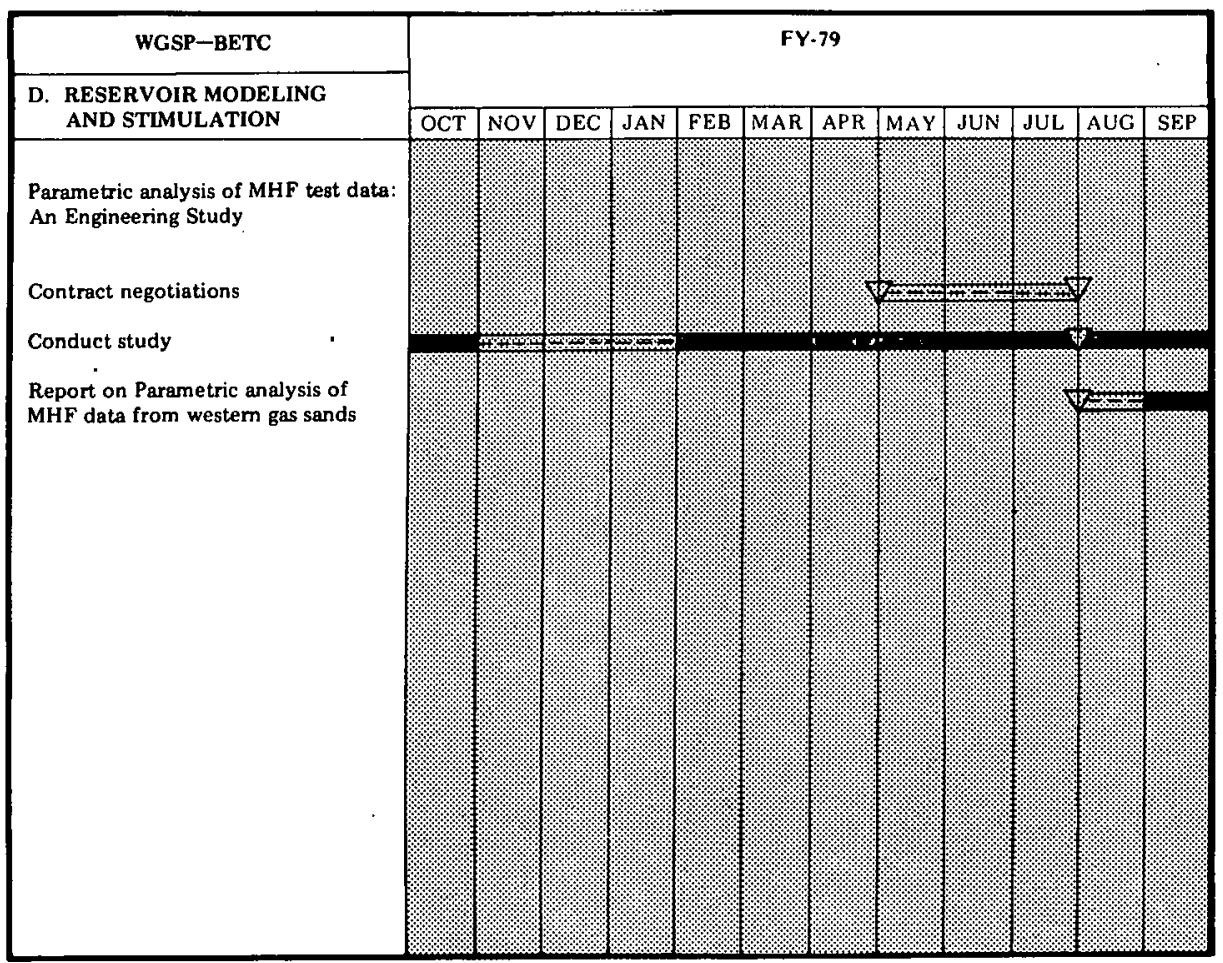

Figure 4-5 Continued 


\subsection{GAS RESEARCH INSTITUTE}

The Gas Research Institute (GRI) has initiated their project for field evaluations of reservoir fracturing to increase producibility of tight gas reservoirs by providing essential field data. GRI will coordinate their proposed work with DOE-funded projects to increase the efficiency, costeffectiveness and scope of research in critical WGSP-related areas of resource characterization and stimulation technology. Three projects were funded in July and work is proceeding.

\subsubsection{Hydraulic Fracturing and Testing of Rio Blanco Natural Gas Company No. 397-19-1 Government Well}

The reservoir analysis of the Rio Blanco Natural Gas Company No. 397-19-1 Government well continued. The well was blown down on September 5 in preparation for a new set of perforations. Prior to perforation, the well was flowing through a 1/16 in. orifice at 1-2 psi after approximately two hours of flow (the well had a stabilized flow).

The tubing was entered at $0900 \mathrm{hrs}$ on September 9 with a 1-11/16 in. magnetic decentralized, retrievable, hollow, carrier-shaped charge type gun and casing collar locator (CCL). Attempts to tie the CCL to the existing Gamma/Bond Log/CCL at the bottom of tubing proved fruitless since the packer had been set deeper than reported and below the lowermost collar recorded on the bond log. Accordingly, a Gamma/CCL tool was delivered to location. The perforating control strip was recorded on depth with the Compensated Density/Compensated Neutron/Gamma Log. The well was shot select-fire at one-foot intervals from 8,503 through 8,506 and 8,554 through 8,556 (Density-Neutron depths in feet).

After perforating the lower zone, the well was allowed to flow through the $1 / 16 \mathrm{in}$. orifice for $30 \mathrm{~min}$. Pressure was gauged at $4.5 \mathrm{psi}$ flowing tubing pressure. The upper zone was then perforated. The well was shut in except for the flow through the 1/16 in. orifice and within two hours the pressure was $10.5 \mathrm{psi}$.

Nitrogen cleanup of the well enhanced production to a stabilized rate of 13 MCFD. The well was shut in on September 13 to perform a BHP buildup test. The test, conducted from September 13 to September 19, indicated approximately the same flow capacity, $\mathrm{kh}$, as derived from the previous two tests in July. This wasipostulated from a final flow capacity value of $20 \mu \mathrm{d}$-ft.

A breakdown was performed September 20 using approximately 10,000 gal 15 percent MSR acid. Leakage from the casing necessitated pulling the tubing and RTTS packer prior to swabbing.

\subsubsection{Evaluation of Seismic Data for Detailed Stratigraphic Studies of Lenticular Sands}

A project has been funded to perform seismic research using recently developed techniques to determine if the geometry and character of lenticular sands at depth can be determined. Sandia Laboratories has proposed to obtain data in the Uinta Basin that will complement and extend outcrop and core hole studies being made under contract to DOE. 
A contract has been negotiated with Seismic International Research, Inc. to perform a twelve-fold survey in the eastern Uinta Basin, Utah. This survey will use an existing core hole and existing outcrop control to delineate sand lenses to a depth of $500 \mathrm{ft}$.

Surveying of shot point and geophone locations continued during the month of September but the seismic crew assigned to the project remained unavailable due to prior commitments.

\subsubsection{Coring and Logging of Pacific Transmission Supply Company No. 22-12 Federal Well}

GRI-funded field operations were delayed until October. (Refer to 3.2 Core Program for a more complete report of this operation).

\subsection{LAWRENCE LIVERMORE LABORATORY}

\subsubsection{Theoretical Analysis}

A time dependent finite element model has been used to determine the material overshoot characteristics from a crack which initiates, propagates bilaterally at half the dilatational wave speed, and stops when one tip reaches an interface. The problem geometry is shown in Figure 4-6. The final crack half length and the time interval from crack starting to stopping is denoted as $c$ and $t_{d}$, respectively. The problem is solved in plane strain and the coordinate system is referenced to the center of the crack. The densities of the two materials are set equal $\left(2.7 \mathrm{gm} / \mathrm{cm}^{3}\right)$, and the Lame constants for each material are also equal so that Poisson's ratio is 0.25 for both materials.

Calculation results are shown in Figure 4-7. Nondimensional vertical displacement, v/c, is plotted versus nondimensional time, $t / t_{d}$, for the point $x=0.8 \mathrm{c}, \mathrm{y}=0.1 \mathrm{c}$. Three calculations were made, corresponding to three sets of values for the Lame constants as shown in Figure 4-7. The constants $\mu_{1}$ and $\lambda_{1}$ were kept at $30 \mathrm{GPa}$ for all three calculations so the center curve corresponds to the homogeneous case. Points of maximum displacement are related to the maximum likelihood of crack reinitiation. Interface penetration becomes less likely and peak displacement occurs earlier as the second material becomes stiffer.

The equilibrium model was applied to analyze some of the effects of frictional interfaces on a pressurized fracture as the crack propagated toward the interface. As reported in the August WGSP Status Report, three calculations were performed with these different frictional stresses applied along the interface. The geometry of the fracture and the interface used in the calculations are shown on Figure 4-8. The pressure in the crack was constant, and the material on both sides of the interface was identical with a Poisson's ratio equal to 0.25 . The ratio of the pressure in the crack to Young's modulus for the material $\left(\mathrm{P}_{c} / E\right)$ was $1.5 \times 10^{-3}$. In these calculations, the effect of changes in pore pressure due to leakage of fluid from the crack into the surrounding material was ignored. 


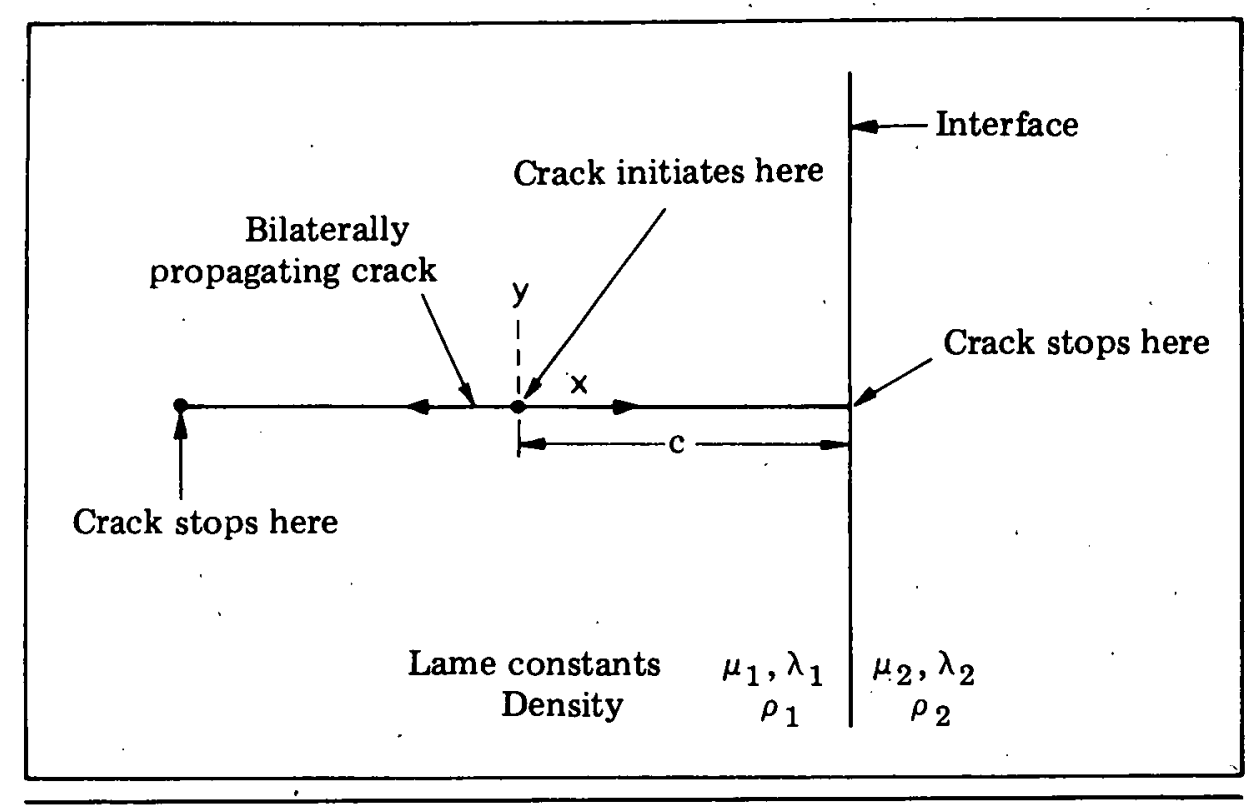

Figure 4-6 Geometry of Time Dependent Crack Problem

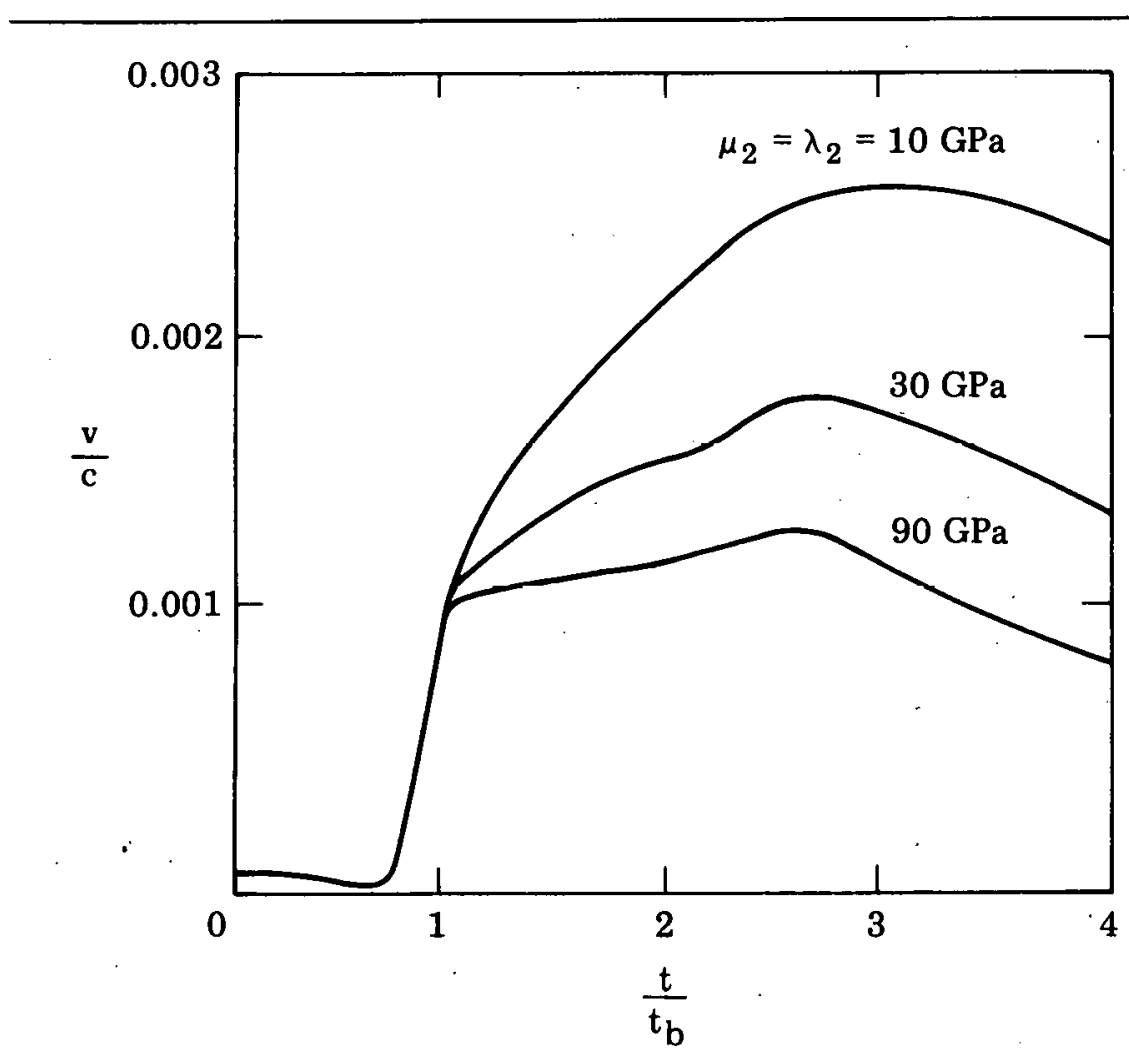

Figure 4-7 Displacement in $Y$ Direction of Point $x=.08 c, Y=.01 c$. The Elastic Constant for Material 1 is $30 \mathrm{GPa}$. 


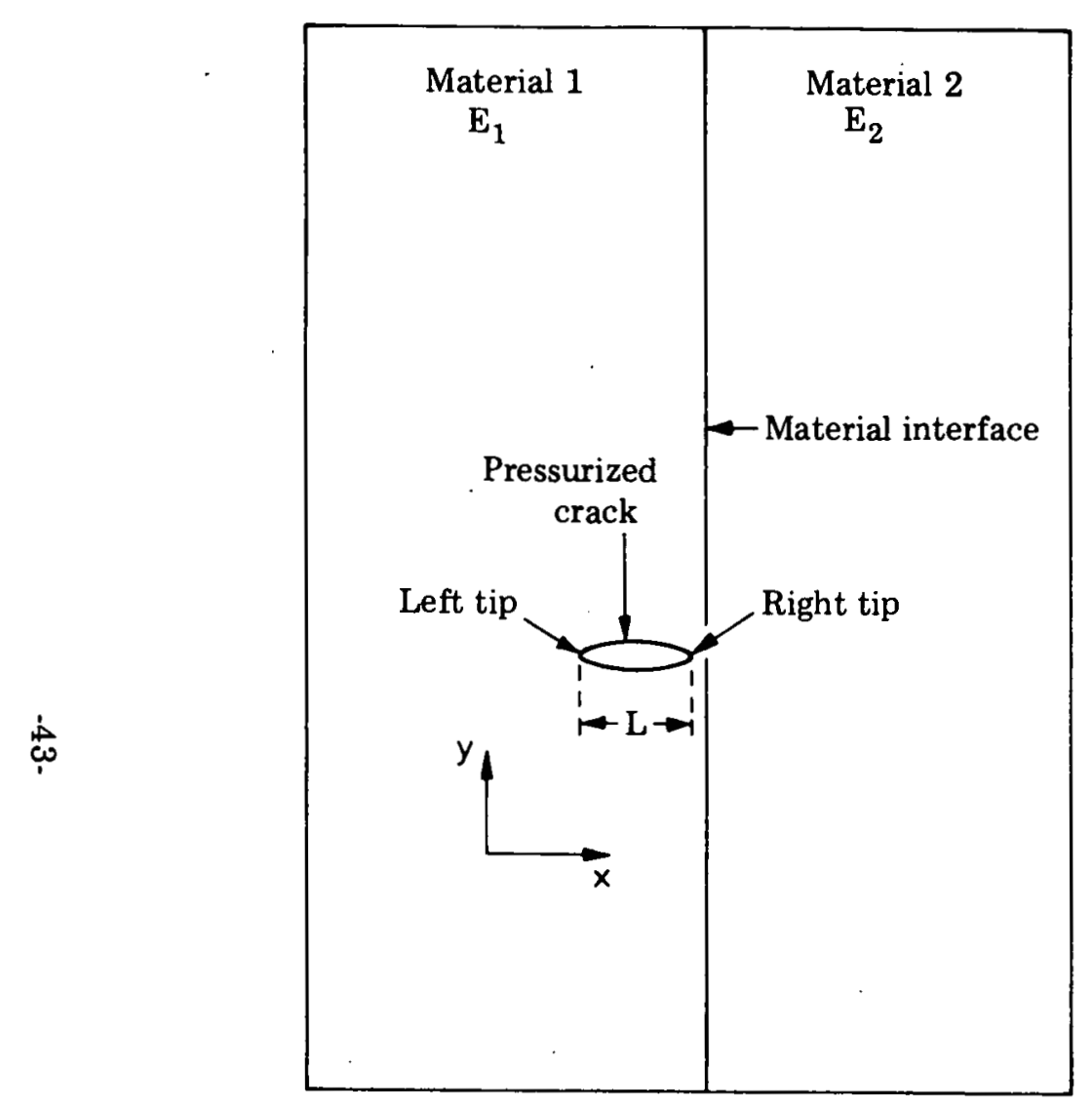

Figure 4-8 Geometry of a Fracture near a Frictional Interface

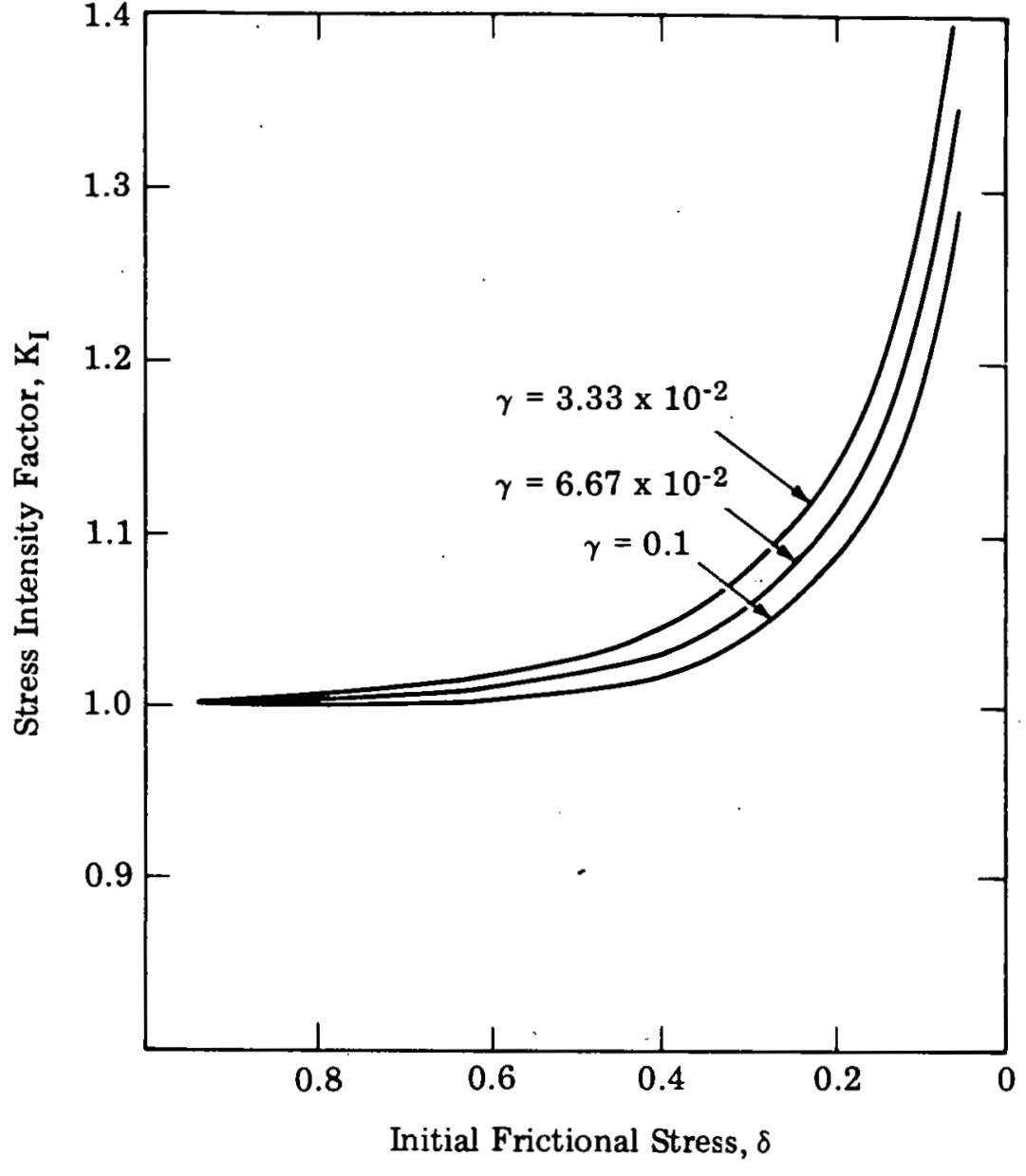

Figure 4-9 Variation in Mode I Stress Intensity Factor as Crack Approaches a Frictional Interface for Variation in Frictional Stress Along the Interface 
The results of the calculations are shown in Figure 4-9 with the distance of the crack from the interface scaled with the crack length. The ratio of the initial frictional stress to the pressure in the crack, $\gamma=\tau_{f} / P_{c}$, for these three sets of calculations was $0.033,0.067$ and 0.1. In these calculations, the crack did not penetrate the interface; therefore the frictional conditions for penetration of the interface have not been evaluated. However, experimental data which can provide correlation for these analyses are being developed. The results presented show how changes in the interfacial frictional stress tends to enhance or impede fracture propagation toward the interface.

When the scaled distance from the crack to the interface is greater than 1 , the effects of the frictional interface on the pressurized crack are small. Relative motion along the interface increased as the pressurized fracture approached the interface and as the frictional coefficient became smaller. The frictional stress along the interface changed as the crack neared the interface. The largest change in this frictional stress occurred in a region directly ahead of the crack tip where it decreased to very small values when the scaled distance was less than 0.2. The mode I stress-intensity factor was scaled (Figure 4-9) with $P_{c} \sqrt{a}$ where $P_{c}$ is the pressure in the crack and $a$ is the semi-major axis of the fracture ellipse. Figure 4-9 shows changes in the scaled mode I stress intensity factor $\left(\mathrm{K}_{\mathrm{I}}\right)$ for the three values of the initial stress on the interface as the crack tip approaches the interface. The stress intensity factor is expressed as the tendency to break, and as this friction increases, the fracture propagation should be enhanced. Figure 4-9 shows that for the lower frictional stresses along the interface, the tendency to break increases more rapidly as the crack approaches the interface. This indicates the crack is more strongly drawn toward the interface for the lower frictional stresses, a similar tendency to when a lower modulus material is to the right of the interface. However, the chance of penetrating the interface decreases with frictional stress comparing with the experimental results.

Formulation had begun on equations which were required to construct a theoretical model for analyzing the flow and pressure distribution in a propagating crack.

\subsubsection{Rock Mechanics Measurements}

Pressure-volume measurements for the Mesaverde shale and sandstone from Rio Blanco County, Colorado, have continued. A protective cover for the high pressure vessel for simultaneous ultrasonic velocity measurements in multiple directions was fabricated.

\subsubsection{Geology/Geophysics}

Compilation and preliminary analyses of data continued on the tectonic environment and geologic structure related to western tight gas sands. A classification can be made based on the tectonic position, stratigraphic thickness and structure, including earthquake seismicity. The Northern Great Plains Province and the Denver, Green River, Uinta, and San Juan Basins each differ according to those criteria. There is some correlation with production histories, reservoir properties, and amenability to fracturing.

\subsubsection{Schedule Status}

Figure 4-10 is a milestone chart depicting LLL WGSP status. 


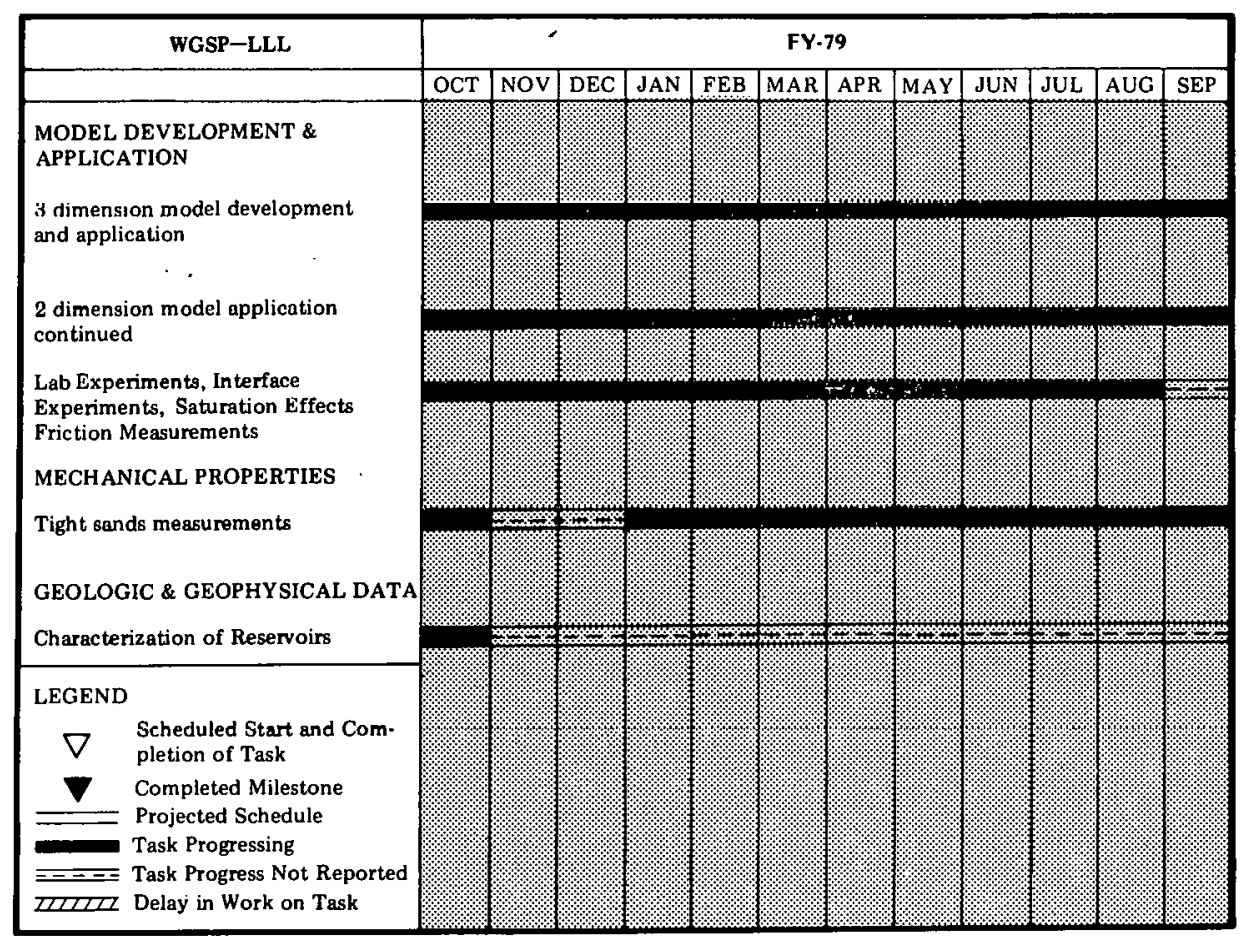

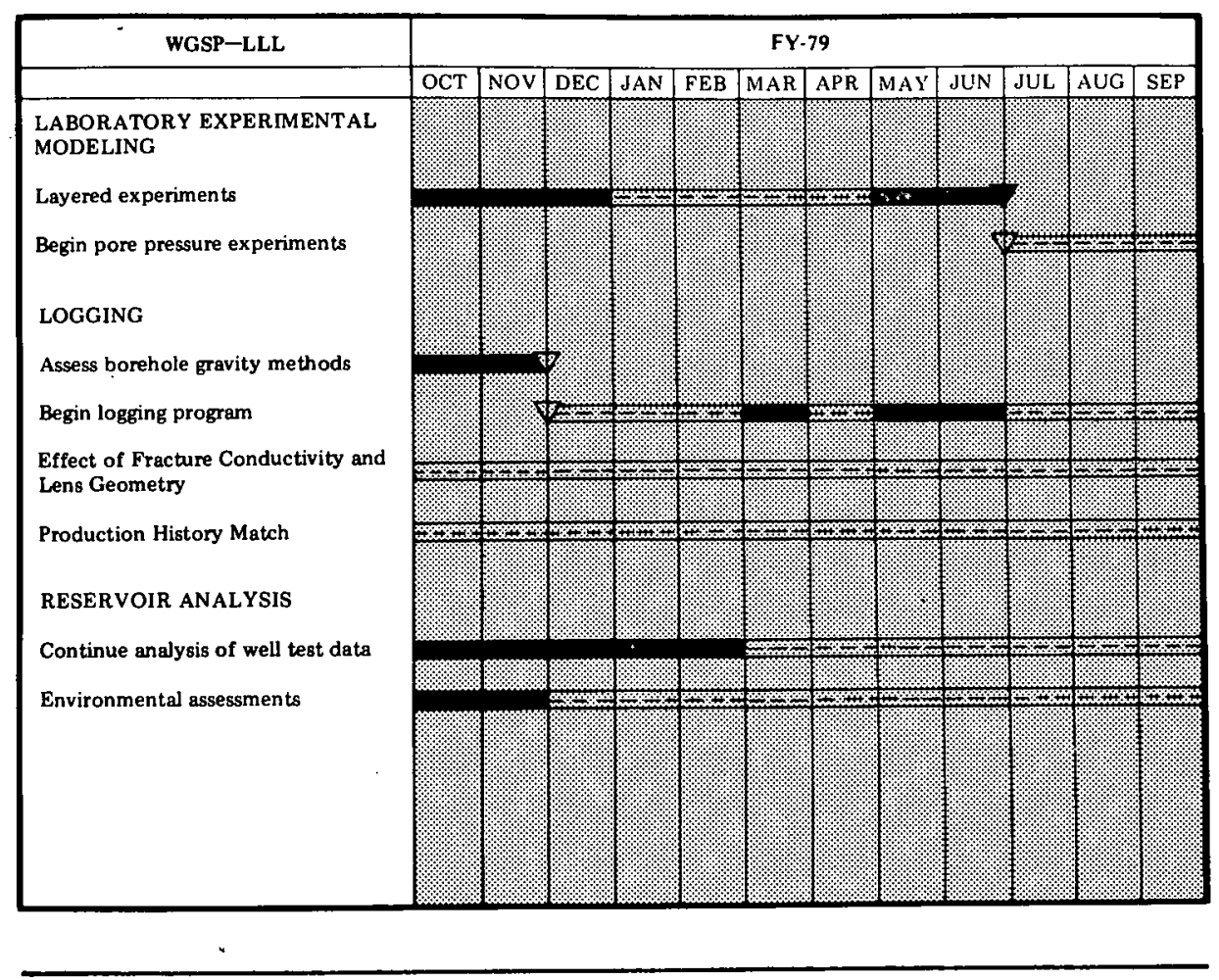

Figure 4-10 Milestone Chart - LLL 


\subsection{LOS ALAMOS SCIENTIFIC LABORATORY}

\subsubsection{Permeability and Porosity Determination on Core Samples}

Laboratory facilities have nearly been completed with the addition of two diamond blade rock saws, a Pistorius cut-off saw, coring drill, chipmunk crusher and centrifugal rock pulverizer, a hydrostatic confining chamber (for pressures up to $10,000 \mathrm{psi}$ ), and an Aminco mercury injection-porosimeter (pressures up to $5,000 \mathrm{psi}$ ).

A study of the microstructure and dynamic behavior of various western gas sands core samples has been initiated. By elucidating the physical structure of these reservoir rocks, as well as their response to varying conditions of temperature and pressure, the macroscale behavior of fluids present in these formations can possibly be better understood and predicted. Scanning electron and optical microscopy will be the principal analytical method. used.

An experimental program to study the relationship between the microstructure and the bulk permeability and porosity of a given formation has been designed to determine the importance and influence of flow through a rock's porous fabric versus flow along fracture channels. It will consist of:

- Microstructure Analysis

- Compression/Extension Dynamics

- Thermal Behavior

\subsubsection{Microstructure Analysis}

A systematic study of the microstructure of tight gas sand reservoirs utilizing a scanning electron microscope (SEM) should provide an understanding of what is responsible for porosity and permeability in a reservoir of this type. Investigations will include pore geometry and pore connection characterization, and attempts to correlate microstructural features (intergranular recrystallization, pore clogging by microparticles, etc.) with the bulk porosity and permeability of samples.

\subsubsection{Compression/Extension Dynamics}

As a rock is gradually compressed, changes in pore volume and fracture flow geometry occur and tend to decrease the porosity and permeability of the medium. Conversely, when a rock is subjected to progressively larger tensional stresses, microcracks will begin to form within or between grains. The density of these microcracks will increase gradually and these voids will begin to form themselves into arrays. Ultimately, an array of cracks will become large enough to satisfy macroscopic conditions for catastropic growth, and fracture failure will occur. 
It is known that increasing in situ lithostatic pressure causes a continuous decrease in formation permeability, but the mechanisms responsible for this decrease are not well known. An explanation of this phenomenon is important to predict flow at depth. Similarly, determining whether rock microstructure controls or influences relatively large scale fracture propagation could lead to insights into well stimulation techniques. Therefore, high magnification, real-time observations of microstructural features (e.g., pore throats, flow fractures) will be made while samples are subjected to varying compressional (ambient to in situ) and tensile (ambient to yield stress) forces.

\subsubsection{Thermal Behavior}

To understand better the role temperature plays in fluid flow through porous media, realtime SEM observations of physical changes occurring in core samples, and measurements of changes in fluid evolution rates will be made while cycling core samples between ambient and typical in situ temperatures.

Currently, an ISI scanning electron microscope $(7 \mathrm{~nm}$ resolution) and several optical and petrographic instruments are available for these studies. as well as transmission electron microscopes and microprobe analysis facilities, should observations or analyses be necessary or desirable. Present activities include the construction of a microscopy laboratory and the development of core sampling procedures and sample preparation techniques that will minimize alterations of natural structure. So the outlined studies can be accomplished, modifications of existing sample stages and designs for specialized specimen stages are currently being formulated.

\subsubsection{Geological Support Studies}

Seven sections of core sample from the Twin Arrow C\&K No. 4-14 well (Piceance Basin) were crushed, pulverized, and analyzed for nitrogen, carbon, hydrogen, and total ash after combustion on the CHN analyzer. Three of the pulverized samples were dried at $100^{\circ} \mathrm{C}$ for 20 hours and compared with samples which were directly analyzed after pulverization. The CHN results showed no statistical differences caused by drying.

Each sample was analyzed at least three times. The following table reports values which represent the averages of all the runs plus the standard deviation.

\begin{tabular}{ccccc} 
Depth (ft) & \% N & \% C & $\% \mathbf{H}$ & $\%$ Ash \\
\hline $1,009.45-1,009.65$ & $0.155 \pm 0.013$ & $3.29 \pm 0.16$ & $0.625 \pm 0.053$ & $88.9 \pm 0.5$ \\
$1,010.35-1,010.50$ & $0.169 \pm 0.023$ & $3.81 \pm 0.40$ & $0.651 \pm 0.081$ & $89.0 \pm 0.3$ \\
$1,012.35-1,012.60$ & $0.226 \pm 0.044$ & $1.95 \pm 0.27$ & $0.362 \pm 0.034$ & $91.0 \pm 0.1$ \\
$1,013.75-1,013.95$ & $0.155 \pm 0.017$ & $1.03 \pm 0.06$ & $0.349 \pm 0.031$ & $94.4 \pm 0.1$ \\
$1,015.60-1,015.80$ & $0.061 \pm 0.094$ & $1.73 \pm 0.13$ & $0.532 \pm 0.023$ & $85.5 \pm 3.4$ \\
$1,016.90-1,017.10$ & $0.054 \pm 0.020$ & $1.58 \pm 0.15$ & $0.517 \pm 0.058$ & $91.4 \pm 1.0$ \\
\hline
\end{tabular}

\subsubsection{Schedule Status}

Figure 4-11 is a milestone chart depicting LASL WGSP status. 

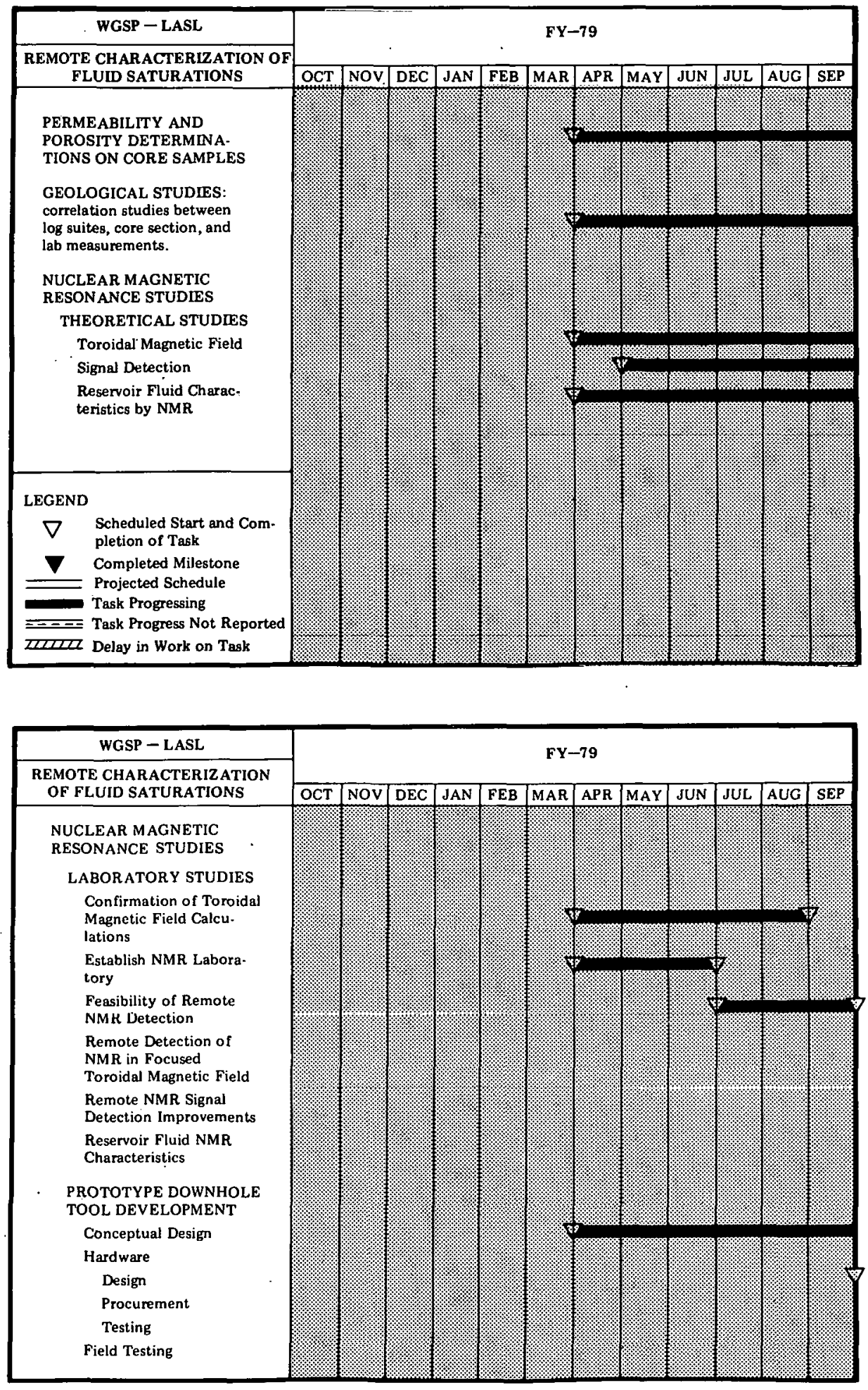

Figure 4-11 Milestone Chart-LASL

$-48-$ 


\subsection{SANDIA LABORATORIES}

\subsubsection{EGR Instrumentation and Diagnostic Program}

\subsubsection{Hydraulic Fracture Characterization}

The PDP 1134 computer is being used for test control and data acquisition with the surface electrical potential system (SEPS). The computer presently contains $32 \mathrm{~K}$ words of memory and will be expanded to $128 \mathrm{~K}$ words. The RT-11 operating system monitor and application programs occupy approximately $16 \mathrm{~K}$ words. The remaining $16 \mathrm{~K}$ words of memory are not adequate to permit the high-speed, long-time duration analog sampling required for instrumentation development. This memory expansion will provide a $96 \mathrm{~K}$ word data buffer and $32 \mathrm{~K}$ words for the RT-11 operating system monitor and applications program.

\subsubsection{Borehole Seismic System}

Preparations for Shell Oil Company's hydrofracture near Lake Texoma are nearly complete. The exact date when fracturing will occur is uncertain due to Shell's undefined preproduction tests. The hydrofracturing will probably begin mid to late November.

A dry nitrogen fracture is being planned October 18 for Weed-Henry's well near Columbus, Ohio. The borehole seismic system will be inserted in the open-hole below the fracture zone $(1,000$ to 1,100 feet $)$ and will monitor the fracturing.

A third fracture experiment is being planned for an Amoco well near Carthage, Texas. The date of this experiment is still uncertain, but it will likely occur in early December.

\subsubsection{Borehole Hydrophone System}

The Edo Western hydrophone system has undergone a second series of tests in the Sandia borehole in Area $\mathrm{Y}$ to determine if the reflections and/or standing wave interference were responsibile for the low formation velocities indicated in August's tests. The test was a repeat of the August one; in addition, the water level in the borehole was raised from a level of $142 \mathrm{ft}$ from the surface to $10 \mathrm{ft}$. Data from the vibroseis source and hammer blows were recorded. Although thorough analysis of the data has not been made, preliminary investigation indicates the change in the borehole water level did not appreciably influence the data. The signals received from vibroseis locations greater than approximately $50 \mathrm{ft}$ from the borehole were too weak to be useful because the power output from the vibroseis was low. A series of tests are being scheduled for the Carlsbad area, where deeper boreholes and a more powerful vibroseis are available.

Both the hardware and the electronics required to assemble and test a 30 -ft hydrophone system which uses the ITC sonic transducer have been received. Assembly and testing of this unit should be completed in the near future. 


\subsubsection{Nuclear Magnetism Logging}

Core analysis is continuing on the 11 samples taken from CIGE well No. 21 in the northeastern Uinta Basin. To date, NMR testing of these samples has not started.

\subsubsection{Formation Mapping}

Topographic surveying and seismic data acquisition have been delayed because of crew unavailability. Mobilization to site No. 1 is expected to take place in October.

\subsubsection{Schedule Status}

Figure 4-12 is a milestone chart depicting Sandia's progress in WGSP activities.

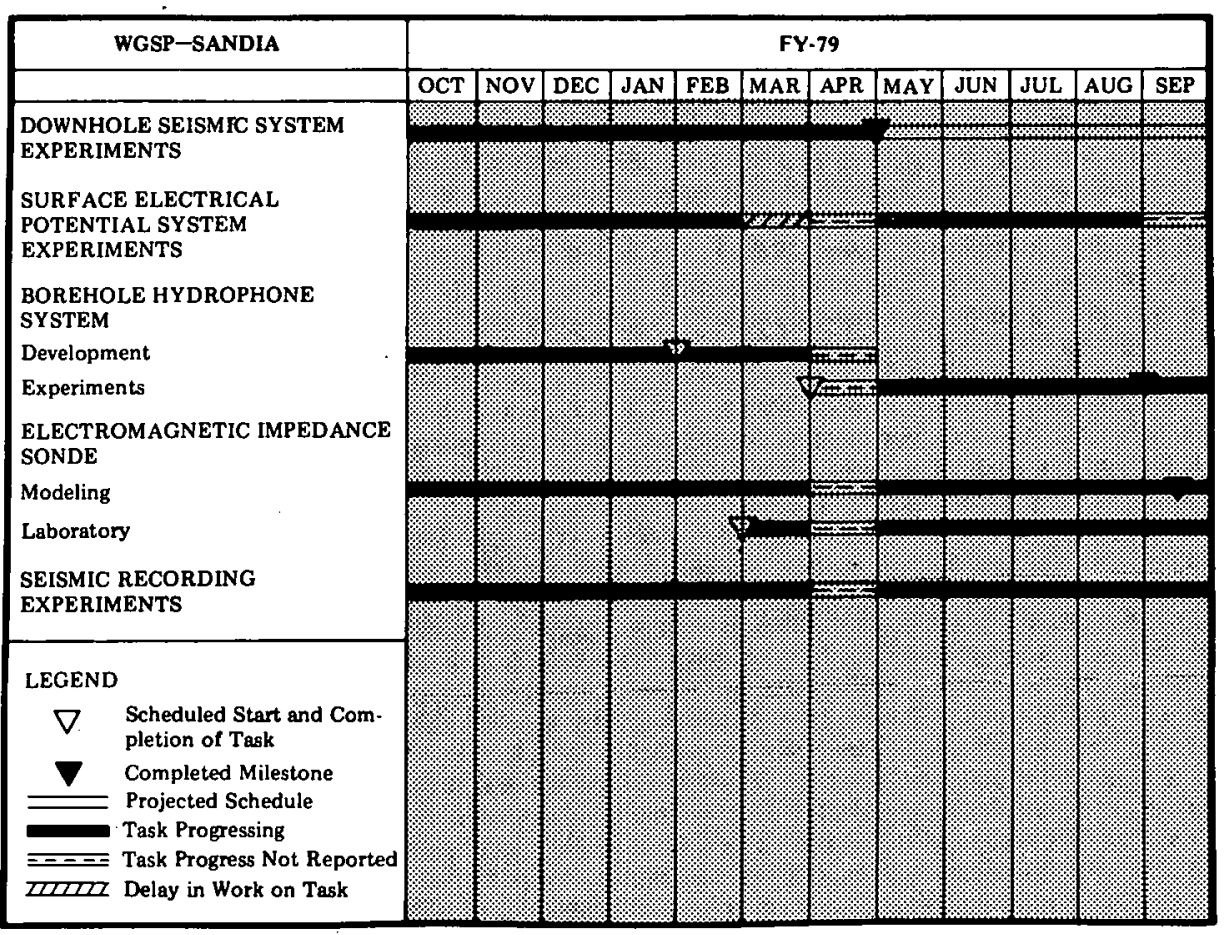

Figure 4-12 Milestone Chart-Sandia 


\section{FIELD TESTS AND DEMONSTRATIONS}

\subsection{BACKGROUND}

Field tests are essential to verify the findings of laboratory tests and modeling studies. The field test and demonstration program involves cooperation between industry and govern. ment and also interacts geologic studies with laboratory research and development. The following projects are active in the WGSP:

- A dry gas injection experiment in the Wattenberg Field, Colorado, by Colorado Interstate Gas Company,

- MHF demonstrations by Gas Producing Enterprises in the Uinta Basin, Utah,

- MHF treatment of the Cotton Valley Limestone Formation in Limestone County, Texas, by Mitchell Energy Corporation,

- MHF demonstrations in the Piceance Basin, Colorado, by Mobil Research and Development Corporation and Rio Blanco Natural Gas Company, and

- A mineback testing program by Sandia Laboratories.

Table 5-1 summarizes completed and active WGSP MHF treatments. Progress of these projects is presented in the following sections. 
Table 5-1 MHF Contract Location and Frac Data

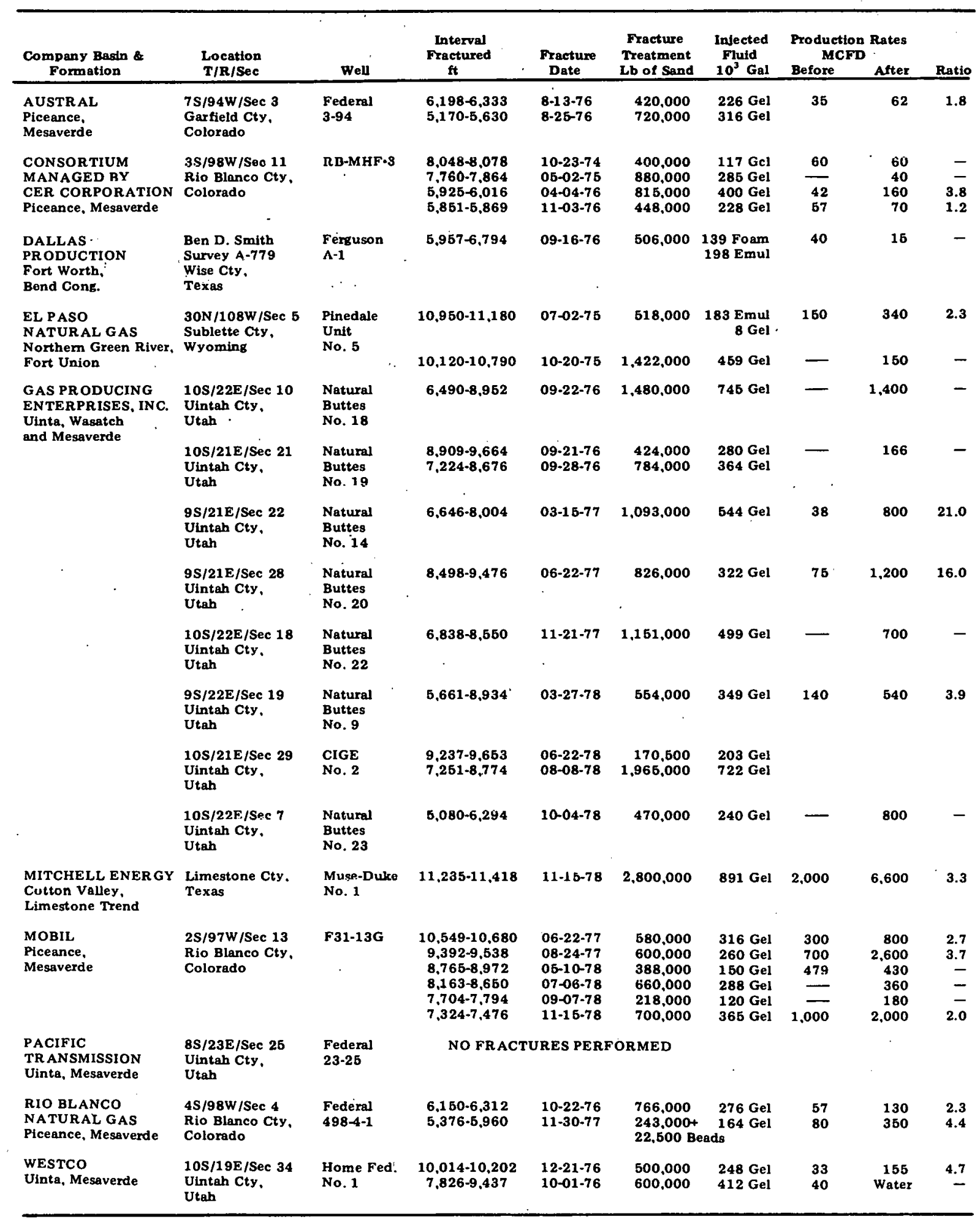




\section{FRACTURING EXPERIMENT}

CER Corporation

Las Vegas, Nevada

Interagency Agreement Date:

Anticipated Completion Date:

Project Cost (estimated):

Principal Investigator:

Technical Advisor for DOE:
June 19,1974

July 1, 1979 (Closing)

DOE ............................. $\$ 1,975,000$

Industry $\ldots \ldots \ldots \ldots \ldots \ldots \ldots \ldots, 1,630,000$

Total .............. $\overline{\$ 3,605,000}$

G. Ruetkehans

C. H. Atkinson

OBJECTIVE

This stimulation experiment is being conducted in low-permeability, massive gasbearing sandstone reservoirs in the Piceance Basin in western Colorado, to test advanced hydraulic fracturing technology where it has not been possible to obtain commercial production rates. This test is located about 1 mile from the 1973 Rio Blanco nuclear stimulation site to permit comparison of nuclear and hydraulic fracturing techniques in this area.

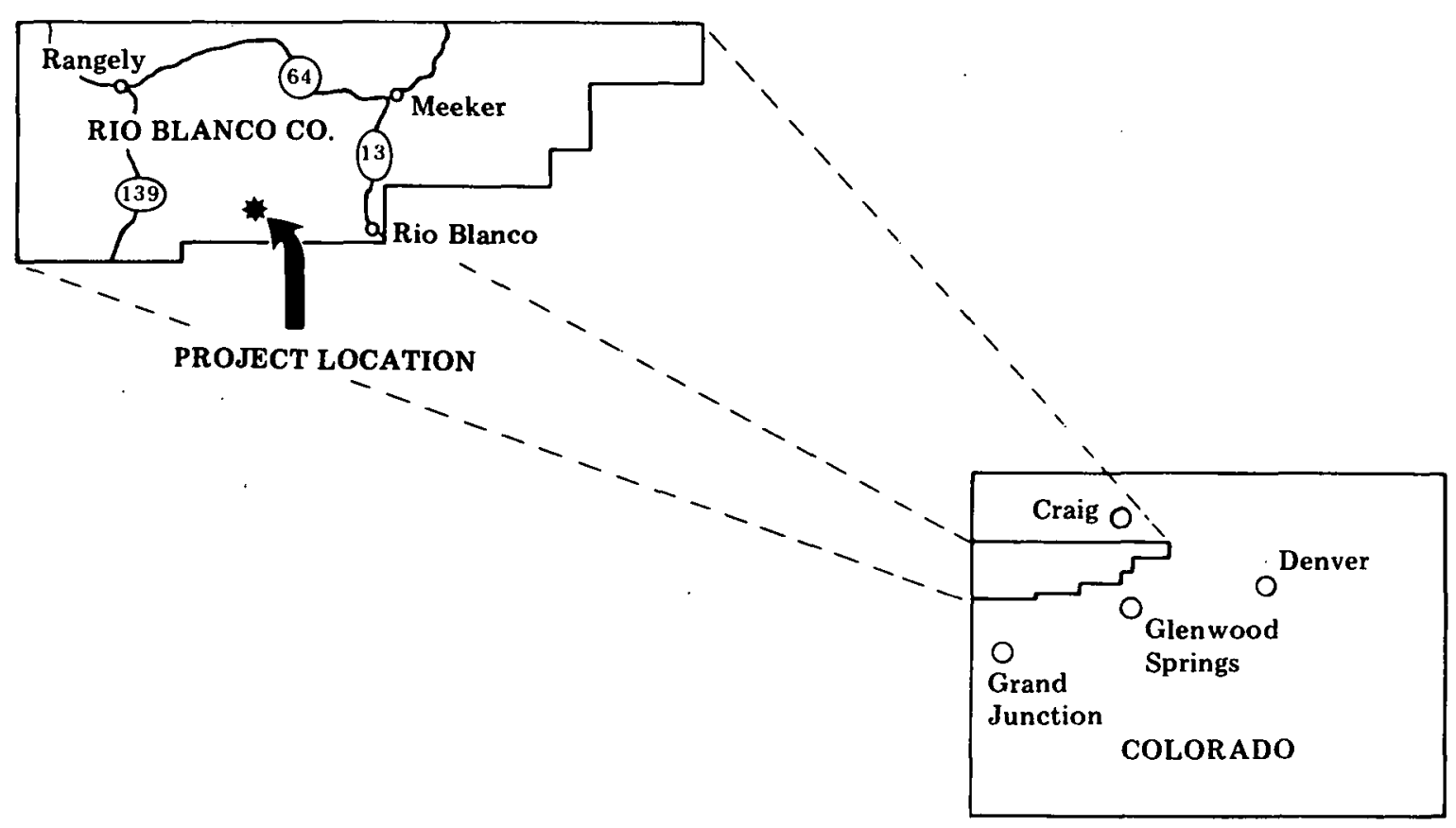




\subsection{CER CORPORATION}

DOE Contract No. EY-76-C-08-0623 was awarded to CER Corporation in March, 1974. The original contract provided for the drilling of a new well and two MHF treatments. Contract modifications added two additional MHF treatments and extended the terms of the contract.

Field activities on the RB-MHF 3 well have been suspended. CER Corporation is transferring the MHF well, to an operator who will attempt to complete the well, commingle the previously fractured zones, test the well and provide the data to the industrial participants and DOE.

The transfer is still pending. 
Colorado Interstate Gas Company

Colorado Springs, Colorado

Contract Date:

Anticipated Completion Date:

September 1, 1977

March 1, 1981

Total Project Cost (estimated):

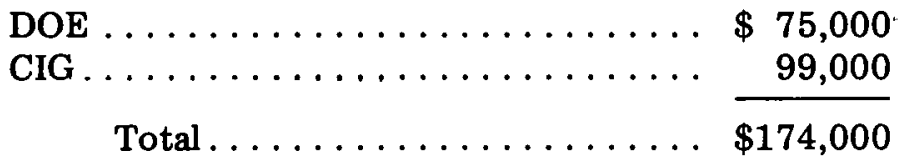

Principal Investigator:

David Wilson

Technical Project Officer for DOE:

C. H. Atkinson

\section{OBJECTIVE}

Cyclic injection of dry natural gas is the method to be used to increase production of tight gas sands.

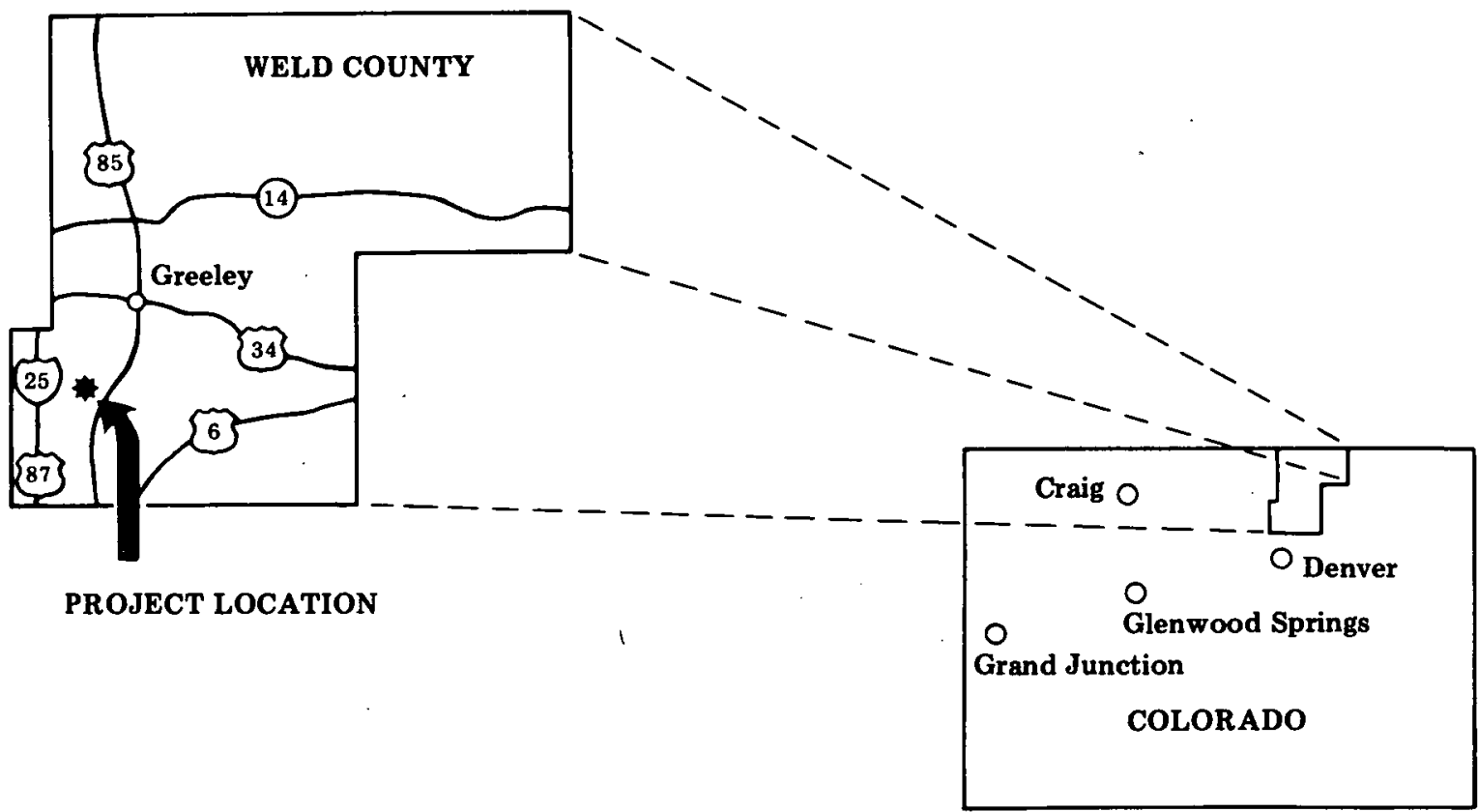




\subsection{COLORADO INTERSTATE GAS}

Cyclic injection and production of dehydrated gas continued at the Miller No. 1 and Sprague No. 1 wells, but with an increasing number of interruptions and compressor shutdowns, primarily due to faulty ball valve operation on the inlet side of the compressor. It appears that conventional gas pipeline valves do not provide the level of consistent operation under the varying pressure and volumetric conditions incurred during cyclic operations. It may be necessary to install valves and/or controls of a different design.

Figures 5-1 and 5-2 illustrate the production and injection volumes and associated pressure of these wells since cyclic operations began.

A table detailing these figures is available upon request. This table also includes the volume of makeup gas obtained from CIG's gathering system, the number of hours the compressor was operating and the amount of fuel used. 

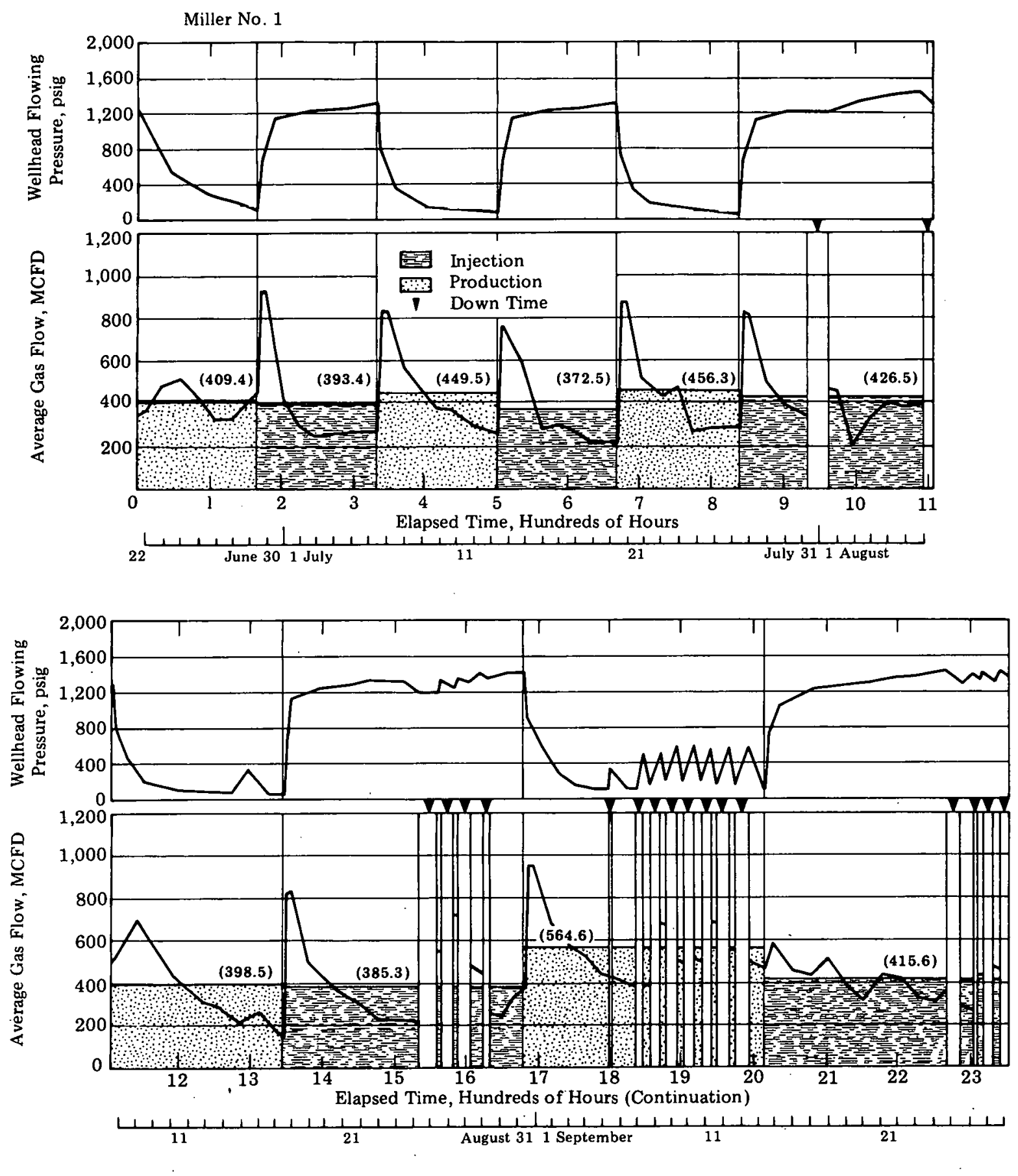

Figure 5-1 Production and Injection Volumes and Associated Pressure of CIG Miller No. 1 

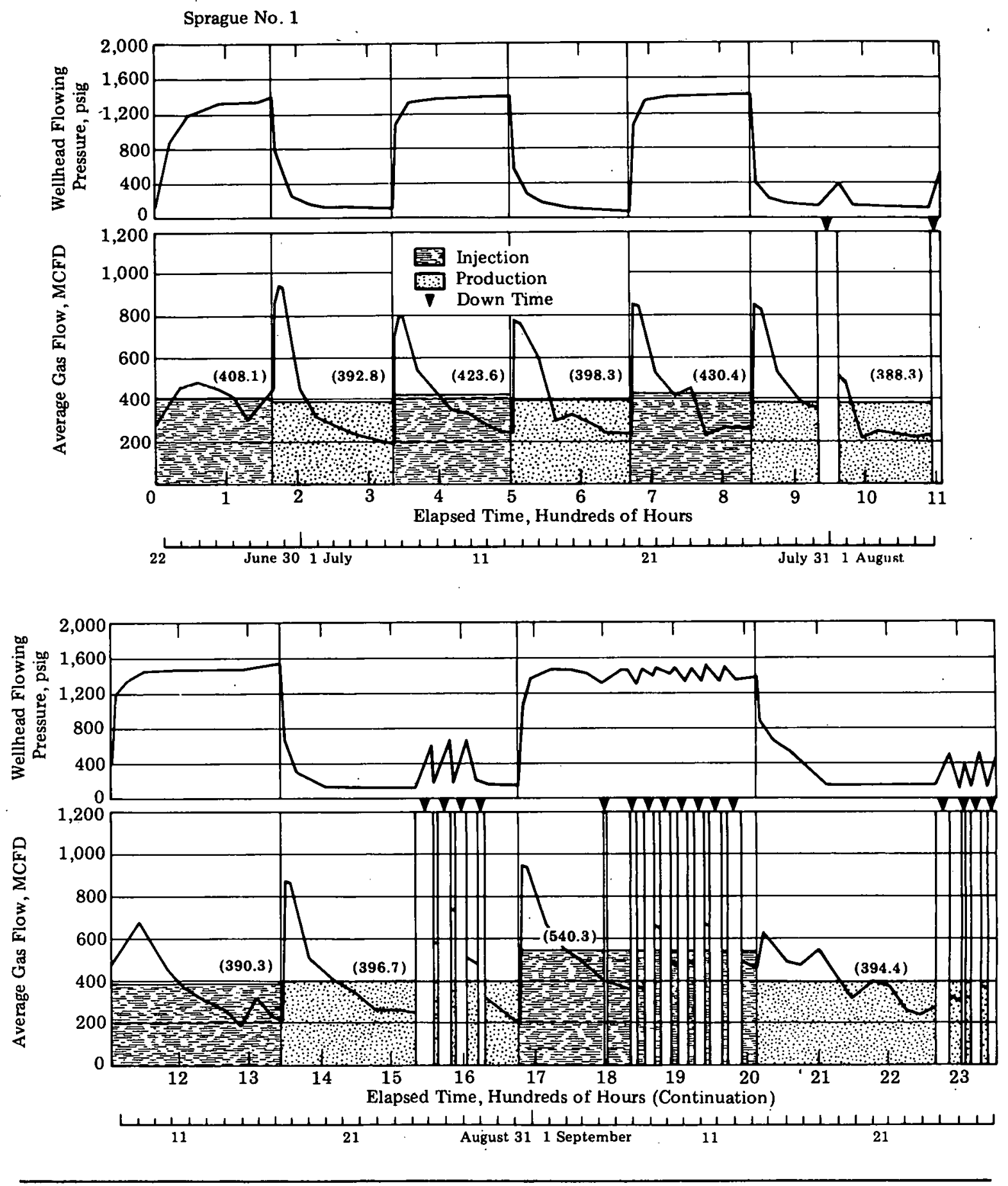

Figure 5-2 Production and Injection Volumes and Associated Pressure of CIG Sprague No. 1 Well 
CER Corporation

Las Vegas, Nevada

Principal Investigator:

Technical Advisor for DOE:
Status: Operational
R. L. Mann

C. H. Atkinson

\section{OBJECTIVE}

The DOE Well Test Facility, consisting of two vehicles, will provide a deep well instrumentation and investigation system to monitor and evaluate the productive potential of all types of wells.

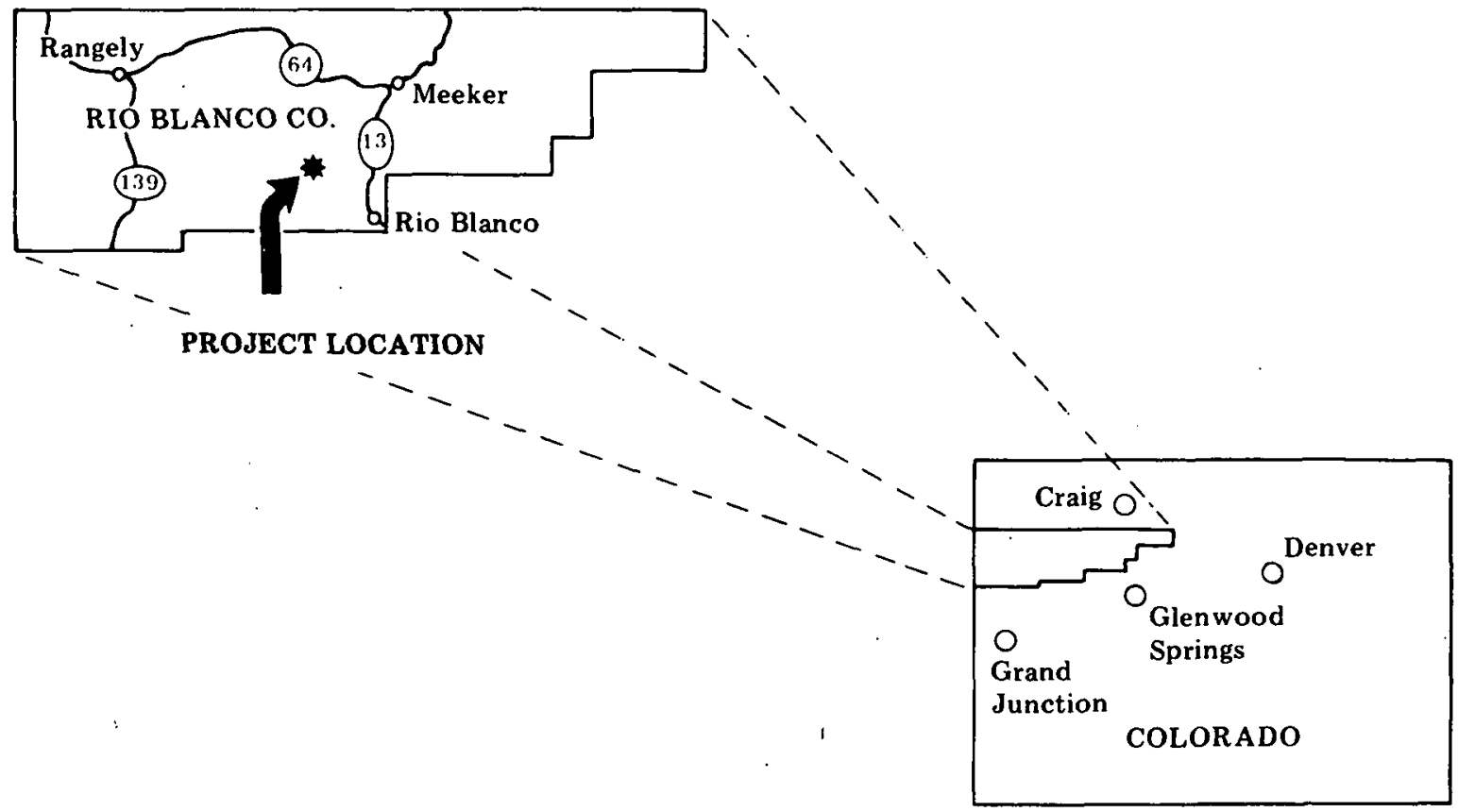




\subsection{DOE WELL TEST F ACILITY}

The DOE Well Test Facility continued to provide technical support to the GRI/Rio Blanco Natural Gas MHF experiment, located in Rio Blanco County, Colorado. During the first part of September, additional perforations were shot, the well cleaned and BHP buildup test conducted. Formation and well test data are tabulated in Table 5-2 and a horner plot of the BHP Buildup data is shown in Figure 5-3.

Greater detail of the activities on the RBNG No. 397-19-1 Government well can be found in Section 4.2.1. 
Table 5-2 397-19-1 Gov't Test Commencing September 13, RBNG

Net Gas Pay $=1.00 \mathrm{ft}$

Well Radius $=.2100 \mathrm{ft}$

Gas Specific Gravity $=.69000$

Carbon Dioxide $=0.0000$ mol pct
Formation Porosity -7.5000

Bottom Hole Temp. $=226.0^{\circ} \mathrm{F}$

Hydrogen Sulfide $=0.0000 \mathrm{~mol}$ pct

Nitrogen $=0.0000 \mathrm{~mol}$ pet

Buildup Test

Flow Time: $73.00 \mathrm{hrs}$.

Flow Rate: 13.00 MCFD

\begin{tabular}{|c|c|c|c|c|c|c|}
\hline $\begin{array}{l}\text { Time } \\
(\Delta \mathrm{T}) \\
(\mathrm{hrs})\end{array}$ & $\begin{array}{c}\text { Horner } \\
\text { Time }\end{array}$ & $\begin{array}{c}\text { Pressure } \\
\text { (psi) }\end{array}$ & $\begin{array}{l}\text { Slope } \\
\text { (psi/ } \\
\text { cycle) }\end{array}$ & $\begin{array}{l}\text { P-Star } \\
\text { (psi) }\end{array}$ & $\begin{array}{c}\mathbf{k h} \\
(\mu \mathrm{d}-\mathbf{f t})\end{array}$ & Skin \\
\hline 66.76 & 2.09 & $1,680.0$ & $3,195.0$ & $2,705.0$ & 13.4 & -2.47 \\
\hline 67.53 & 2.08 & $1,689.0$ & $3,195.0$ & $2,705.0$ & 13.3 & -2.47 \\
\hline 68.26 & 2.07 & $1,696.0$ & $2,727.0$ & $2,557.0$ & 15.6 & -2.55 \\
\hline 68.98 & 2.06 & $1,702.0$ & $2,788.0$ & $2,576.0$ & 15.3 & -2.54 \\
\hline 69.70 & 2.05 & $1,709.0$ & $2,597.0$ & $2,516.0$ & 16.9 & -2.57 \\
\hline 70.43 & 2.04 & $1,714.0$ & $2,853.0$ & $2,596.0$ & 14.9 & -2.53 \\
\hline 71.15 & 2.03 & $1,722.0$ & $2,901.0$ & $2,610.0$ & 14.7 & -2.52 \\
\hline 71.88 & 2.02 & $1,727.0$ & $2,490.0$ & $2,485.0$ & 17.1 & -2.59 \\
\hline 72.60 & 2.01 & $1,733.0$ & $2,530.0$ & $2,497.0$ & 16.8 & -2.58 \\
\hline 73.33 & 2.00 & $1,738.0$ & $2,566.0$ & $2,508.0$ & 16.5 & -2.58 \\
\hline 74.05 & 1.99 & $1,744.0$ & $2,606.0$ & $2,520.0$ & 16.3 & -2.57 \\
\hline 74.78 & 1.98 & $1,749.0$ & $2,419.0$ & $2,465.0$ & 17.5 & -2.61 \\
\hline 76.23 & 1.96 & $1,759.0$ & $2,597.0$ & $2,517.0$ & 16.3 & -2.57 \\
\hline 77.68 & 1.94 & $1,770.0$ & $2,547.0$ & $2,502.0$ & 16.6 & -2.58 \\
\hline 79.13 & 1.92 & $1,779.0$ & $2,356.0$ & $2,448.0$ & 18.0 & -2.62 \\
\hline 80.58 & 1.91 & $1,788.0$ & $2,422.0$ & $2,467.0$ & 17.5 & -2.61 \\
\hline 82.03 & 1.89 & $1,797.0$ & $2,214.0$ & $2,408.0$ & 19.1 & -2.65 \\
\hline 83.48 & 1.87 & $1,804.0$ & $2,130.0^{\circ}$ & $2,386.0$ & 19.8 & -2.66 \\
\hline 84.98 & 1.86 & $1,812.0$ & $2,188.0$ & $2,401.0$ & 19.3 & -2.65 \\
\hline 86.38 & 1.85 & $1,819.0$ & $2,096.0$ & $2,377.0$ & 20.1 & .2 .67 \\
\hline 87.83 & 1.83 & $1,826.0$ & $2,343.0$ & $2,442.0$ & 18.0 & -2.62 \\
\hline 91.45 & 1.80 & $1,845.0$ & $2,289.0$ & $2,428.0$ & 18.4 & -2.63 \\
\hline 95.08 & 1.77 & $1,861.0$ & $2,153.0$ & $2,394.0$ & 19.5 & -2.66 \\
\hline 98.70 & 1.74 & $1,876.0$ & $2,212.0$ & $2,408.0$ & 19.0 & -2.65 \\
\hline 102.32 & 1.71 & $1,891.0$ & $2,262.0$ & $2,420.0$ & 18.5 & -2.64 \\
\hline 105.95 & 1.69 & $1,905.0$ & $2,243.0$ & $2,416.0$ & 18.7 & -2.65 \\
\hline 109.51 & 1.67 & $1,918.0$ & $2,049.0$ & $2,371.0$ & 20.4 & -2.68 \\
\hline
\end{tabular}




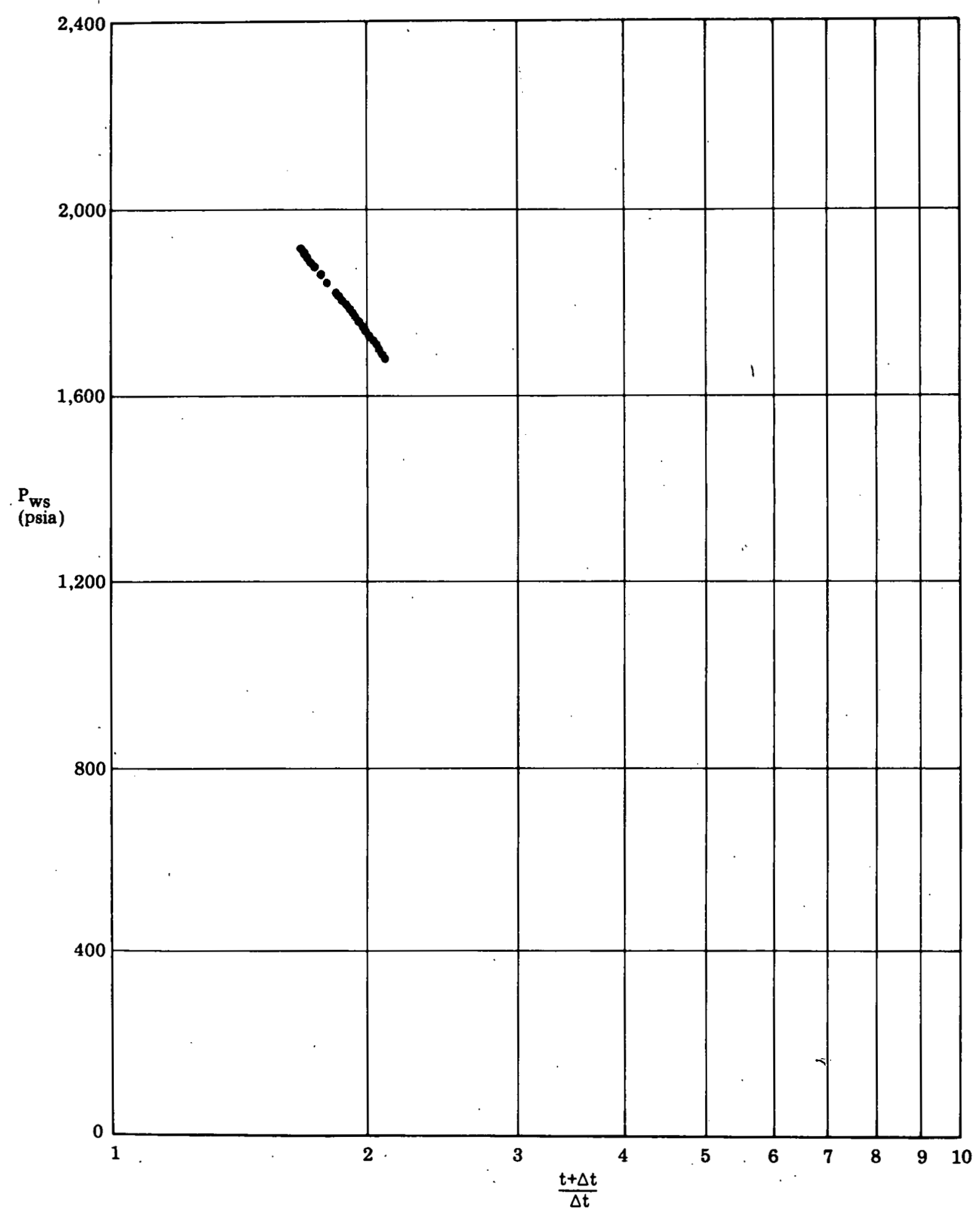

Figure 5-3 Horner Plot for September 13, BHP Test, RBNG-397-1 Gov't 


\section{NATURAL BUTTES UNIT, UINTAH COUNTY, UTAH MASSIVE HYDRAULIC FRACTURING DEMONSTRATION}

Gas Producing Enterprises, Inc.

Status: Active

Subsidiary of Coastal States Gas Co.

Houston, Texas

Contract Date:

Anticipated Completion:

July 1,1976

March 31, 1980

Total Project Cost (estimated):

DOE . . . . . . . . . . . . . \$2,827,000

Industry (prior costs) $\ldots \ldots \ldots \ldots \ldots \quad 1,881,000$

Industry (new costs) . . . . . . . . . . 3,051,000

Total . . . . . . . . . . . $\overline{\$ 7,759,000}$

Principal Investigator:

W. E. Spencer

Technical Project Officer for DOE:

C. H. Atkinson

\section{OBJECTIVE}

To evaluate the effectiveness of massive hydraulic fracturing for stimulating natural gas production from thick, deep sandstone reservoirs having low-permeability.

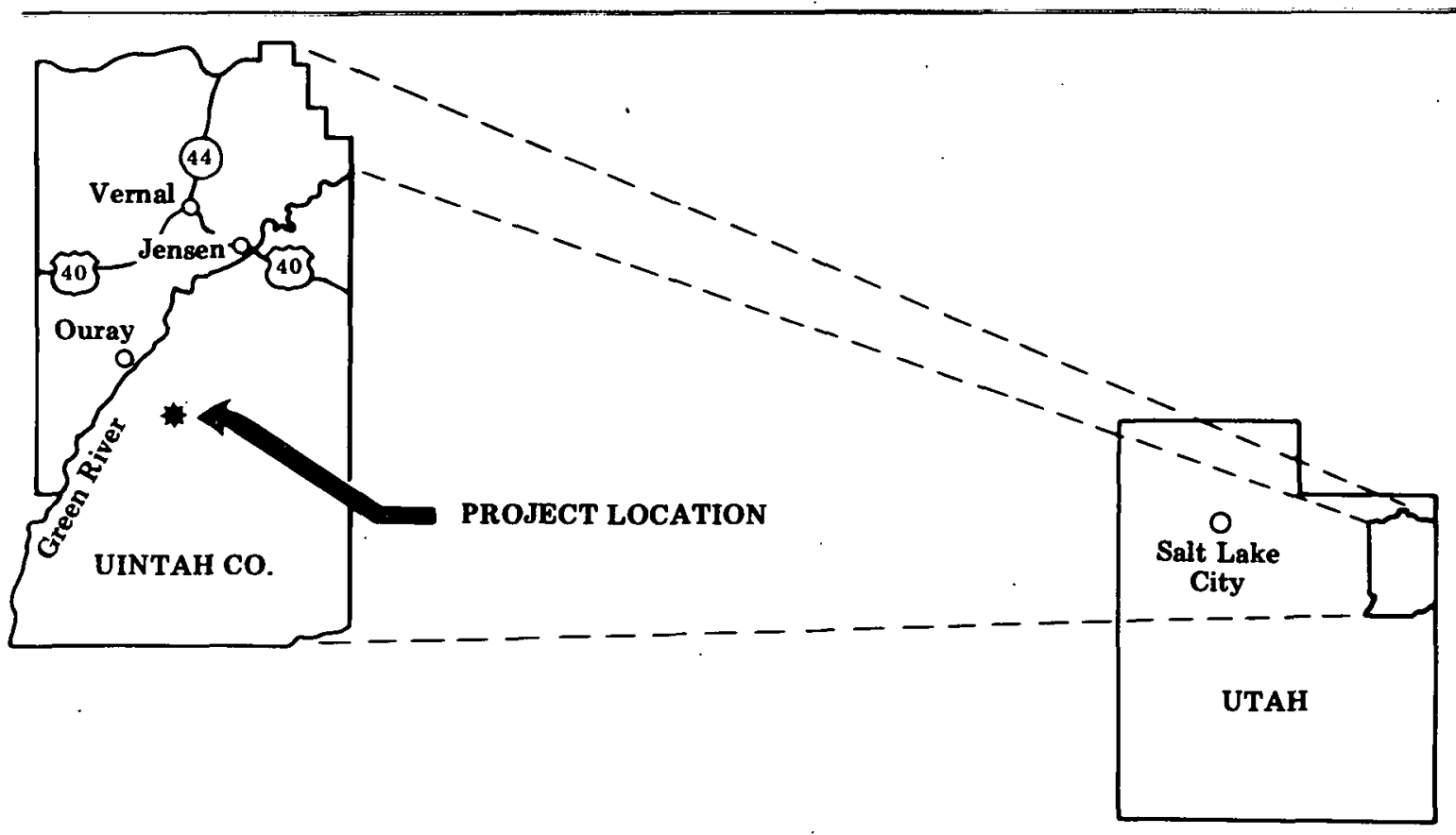




\subsection{GAS PRODUCING ENTERPRISES}

The GPE wells, Naturai Buttes Units $9,14,18$ and 20 flowed to sales during September. Natural Buttes Units 19 and 22 were shut in during the month. NBU 21 was temporarily abandoned in the Mesaverde horizon, but GPE will try to complete it in the Wasatch. Figures 5-4 through 5-9 show production figures.

A comprehensive final report is being prepared. 


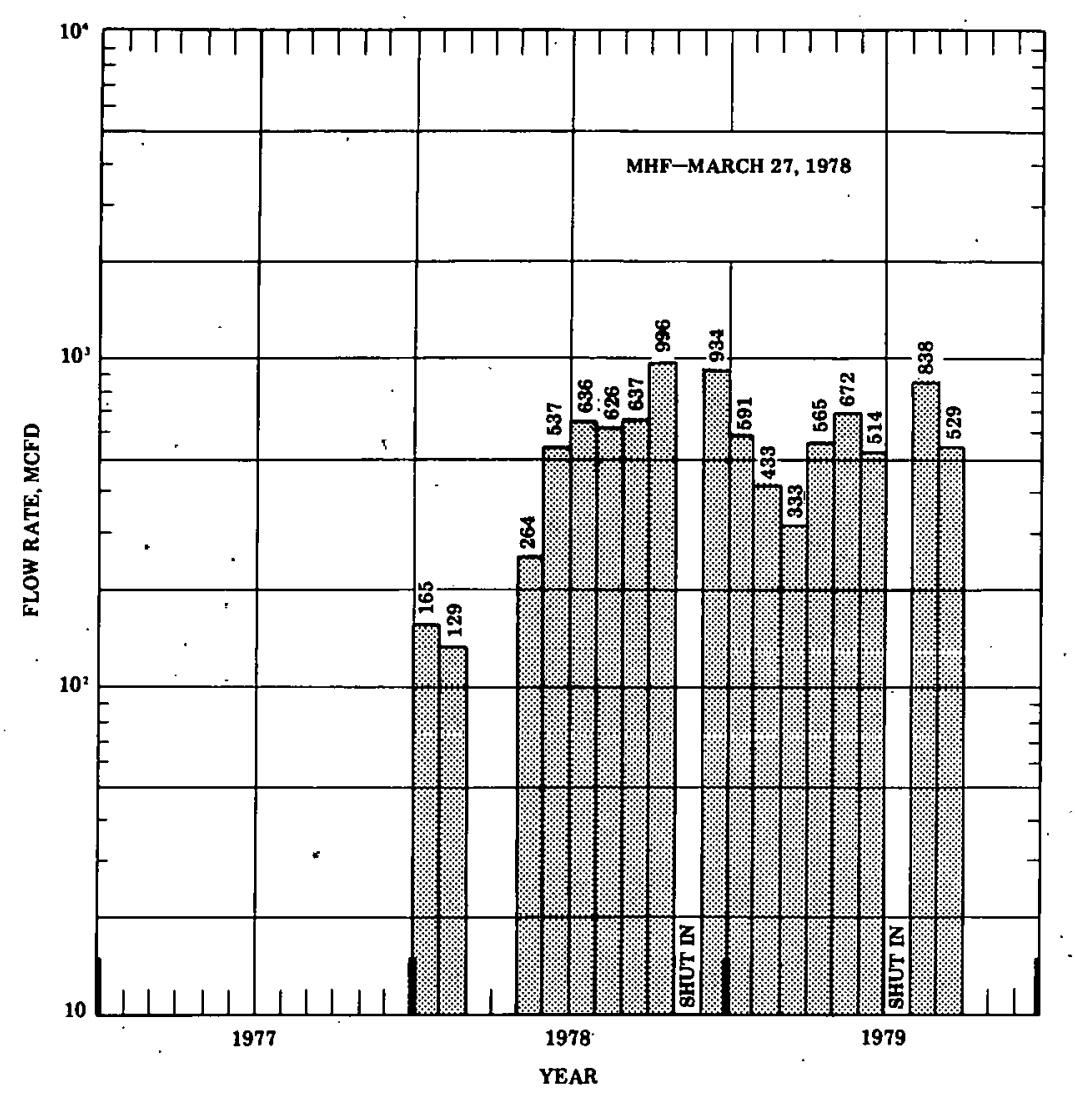

Figure 5-4

Flow Rate Performance of Natural Buttes No. 9 Well

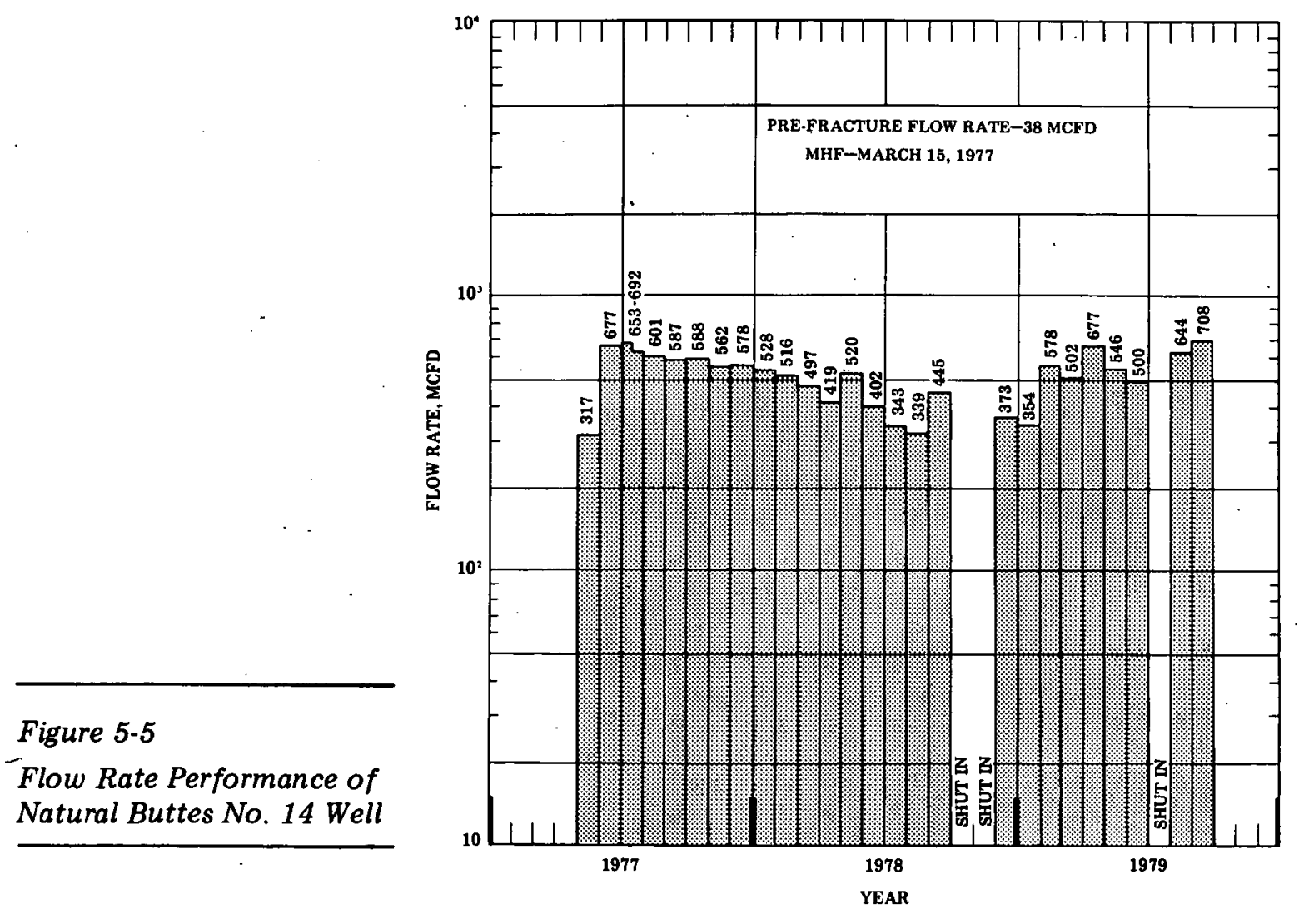



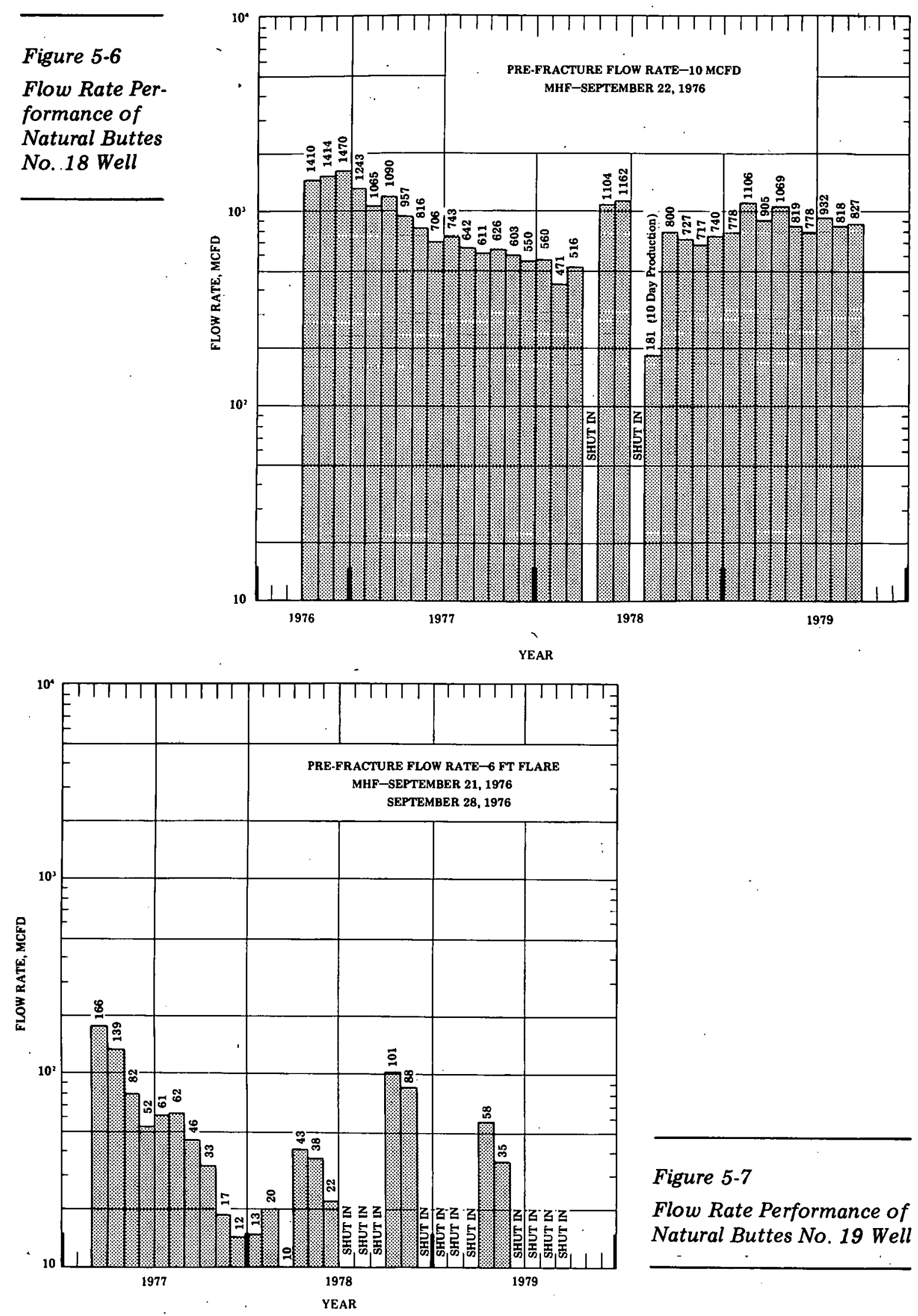

Figure 5-7

Flow Rate Performance of Natural Buttes No. 19 Well 


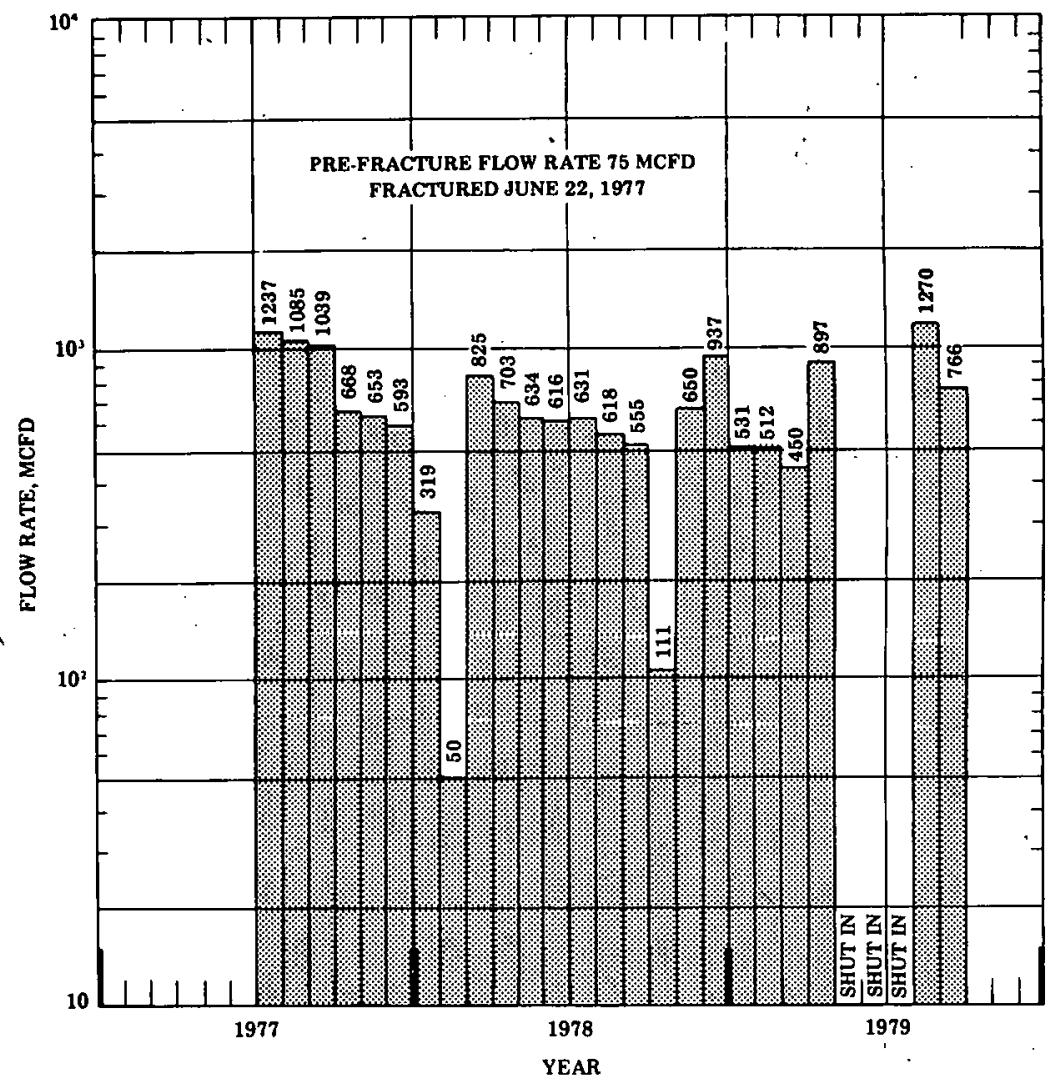

Figure 5-8

Flow Rate Performance

of Natural Buttes No. 20 Well

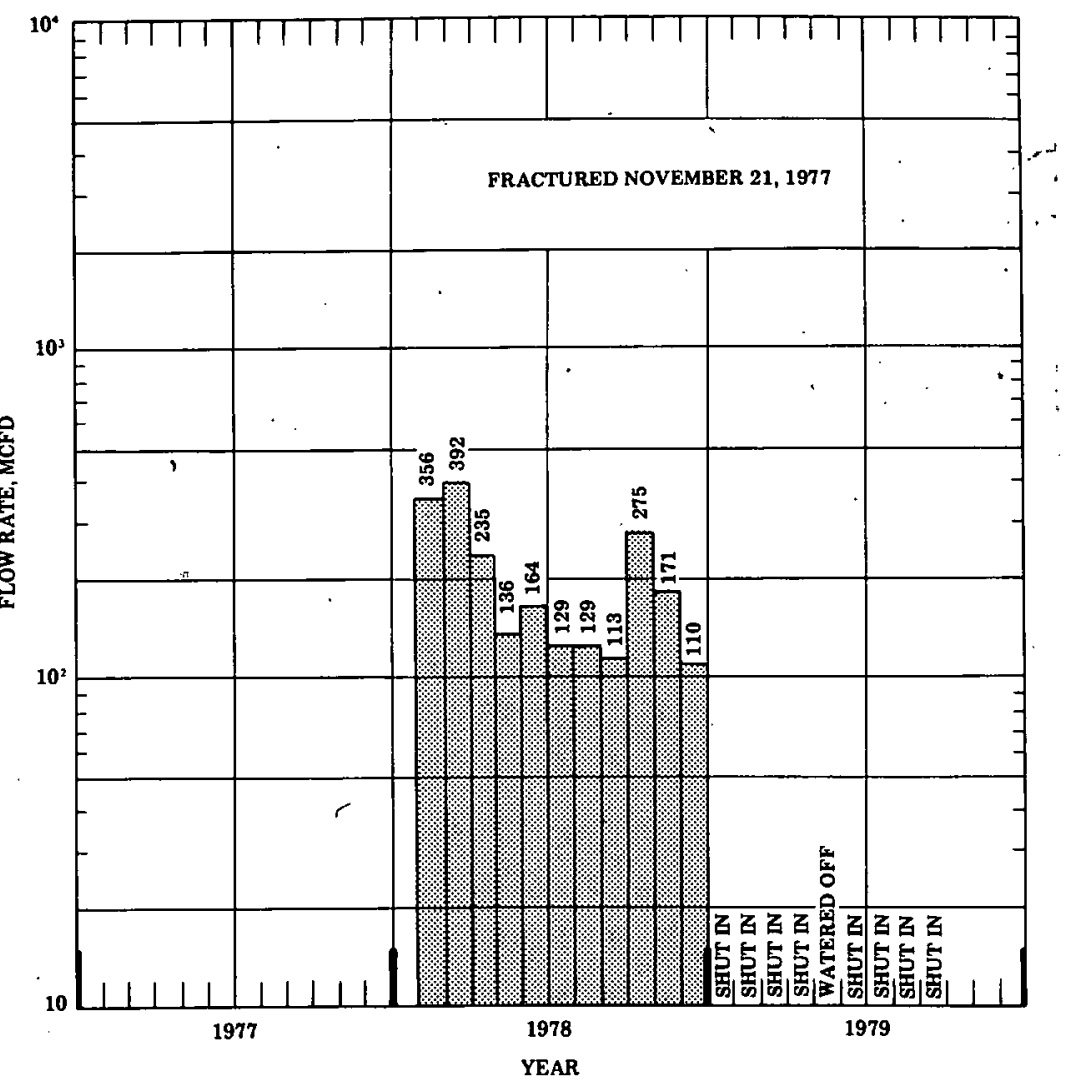


FALLON-NORTH PERSONVILLE FIELD, TEXAS, MASSIVE HYDRAULIC FRACTURING

EF-78-C-08-1547 DEMONSTRATION

Mitchell Energy Corporation

Houston, Texas

Contract Date:

Anticipated Completion:

March 15, 1978

April 30, 1979 (Extension being processed)

Total Project Cost (estimated):

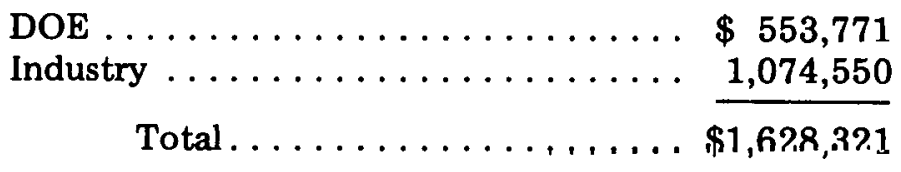

Principal Investigator:

F. D. Covey

Technical Project Officer for DOE:

C. H. Atkinson

Status: Active

\section{OBJECTIVE}

To test massive hydraulic fracturing in the Cotton Valley Limestone Formation.

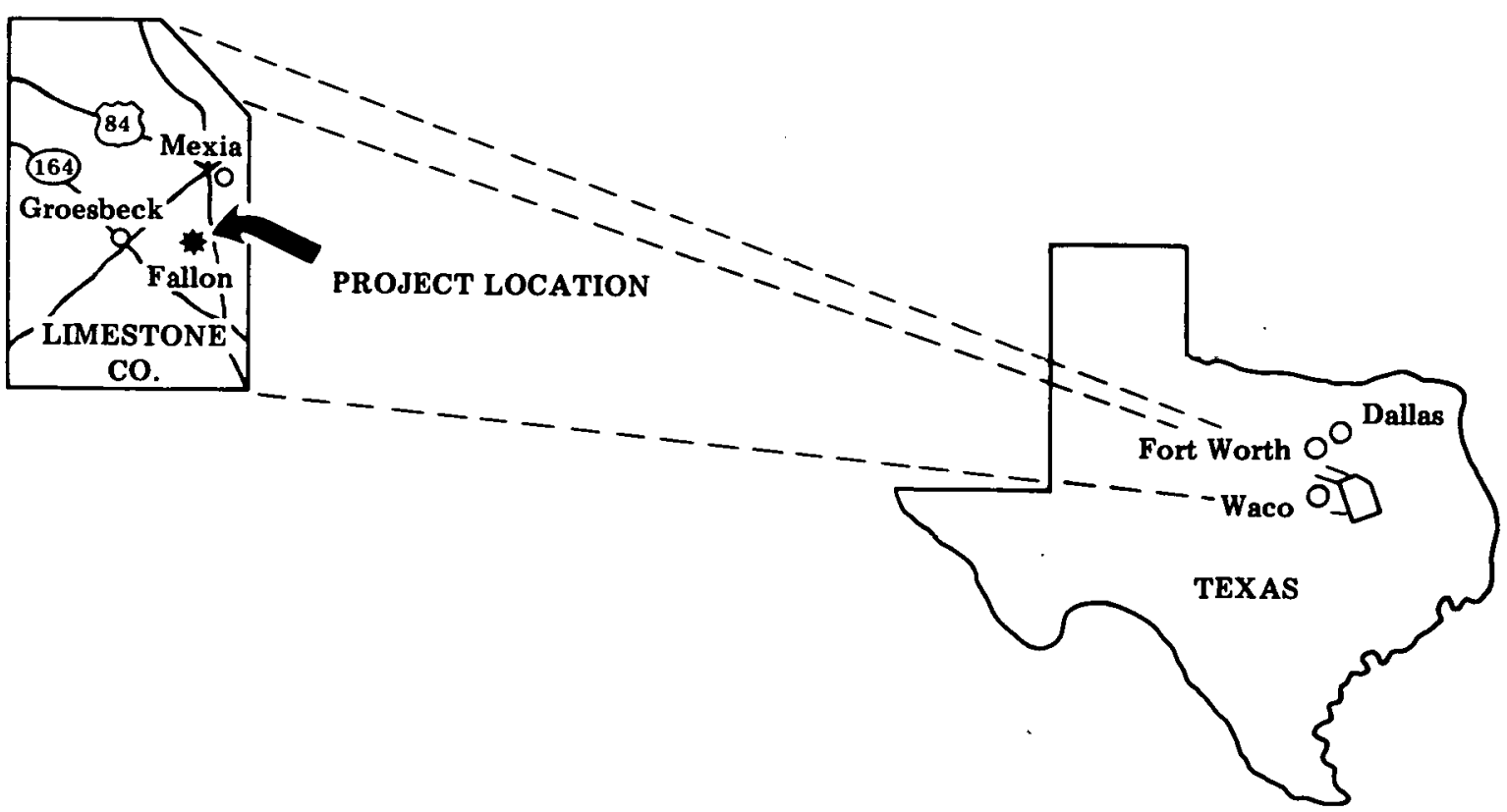




\subsection{MITCHELL ENERGY CORPORATION}

The Mitchell Energy Corporation Muse-Duke No. 1 was opened up on September 5, 1979, after a 28-day shut in period for a pressure buildup survey. The maximum wellhead shut in pressure was 2,590 psig. The initial rate after opening up was 5,700 MCFD and 18 BWPD with a flowing tubing pressure of 1,500 psig. At month's end, the flow rate was 5,100 MCFD and 24 BWPD, with a flowing tubing pressure of 1,800 psig. However, during the month, a series of curtailments occurred, precluding a stabilized flow rate.

Final pressure analysis was delayed due to difficulty in gathering all post pressure data, and some work had to be repeated. The results are expected to be available by the end of October. 
PICEANCE CREEK FIELD, COLORADO,

EY-76-C-08-0678

MASSIVE HYDRAULIC FRACTURING DEMONSTRATION

Mobil Research and Development Corporation

Dallas, Texas

Status: Active

Contract Date:

July 1,1976

Anticipated Completion:

September 30, 1979

Total Project Cost (estimated):

DOE . . . . . . . . . . . . . \$ \$2,510,000

Contractor (prior costs). . . . . . . . 2, 2,376,485

Contractor (new costs) . . . . . . . . 1,590,515

Total. . . . . . . . . . . \$6,477,000

Principal Investigator:

John L. Fitch

Technical Project Officer for DOE:

C. H. Atkinson

\section{OBJECTIVE}

To evaluate the effectiveness of massive hydraulic fracturing for stimulating natural gas production from thick, deep sandstone reservoirs having extremely low-permeability.

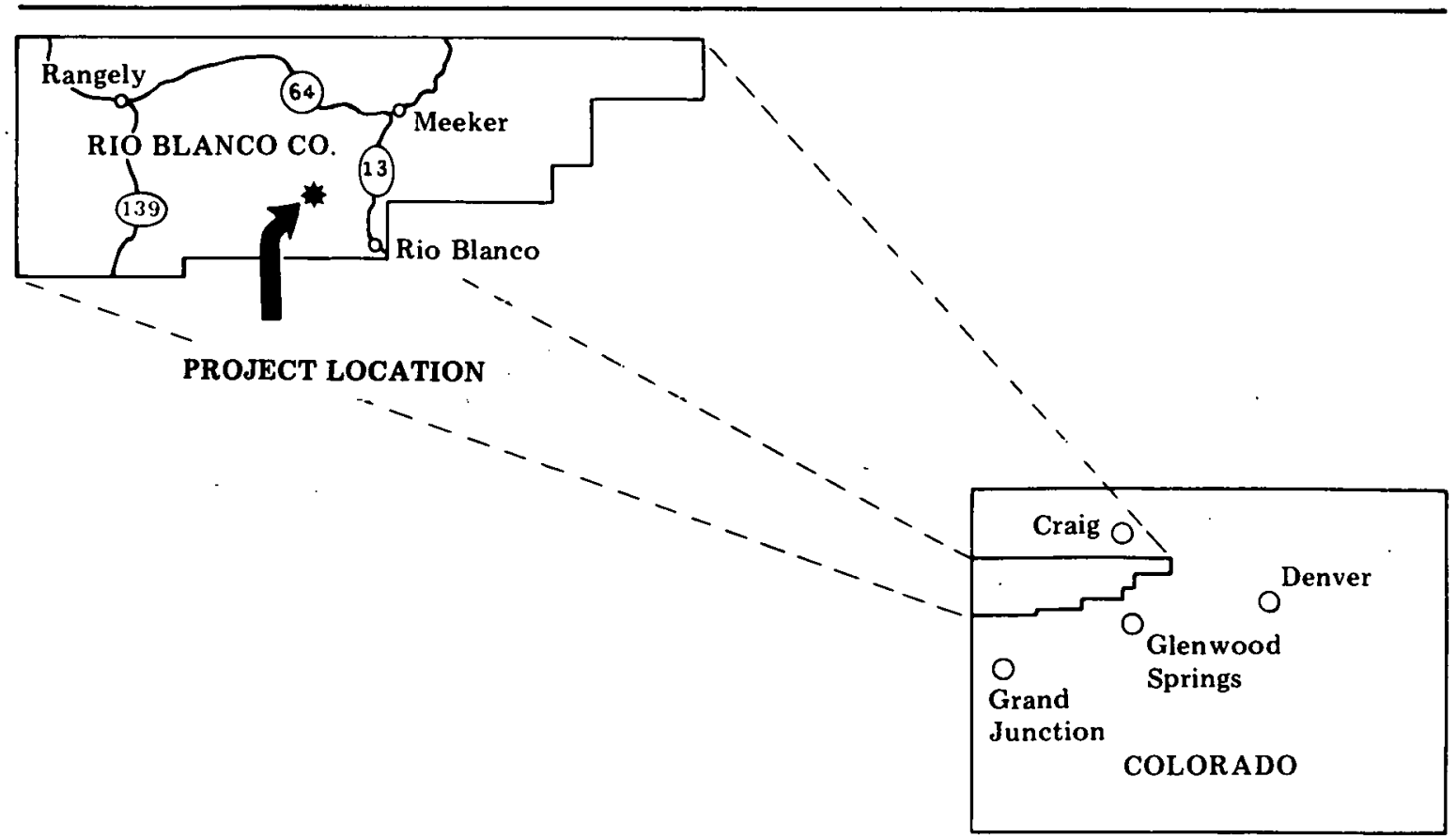




\subsection{MOBIL RESEARCH AND DEVELOPMENT}

A draft of the comprehensive final report was submitted to DOE for comments. The report has been returned to Mobil for printing. 
Denver, Colorado

Contract Date:

Anticipated Completion:

Total Project Cost (estimated):

Principal Investigator:

Technical Project Officer for DOE:
August 1, 1976

July 31,1979

DOE . . . . . . . . . . . . . \$ $\$ 410,000$

Contractor. . . . . . . . . .

Total. . . . . . . . . . . \$1,003,000

Robert E. Chancellor

C. H. Atkinson

\section{OBJECTIVE}

To evaluate the effectiveness of massive hydraulic fracturing for stimulating natural gas production from thick, deep sandstone reservoirs having extremely low permeability.

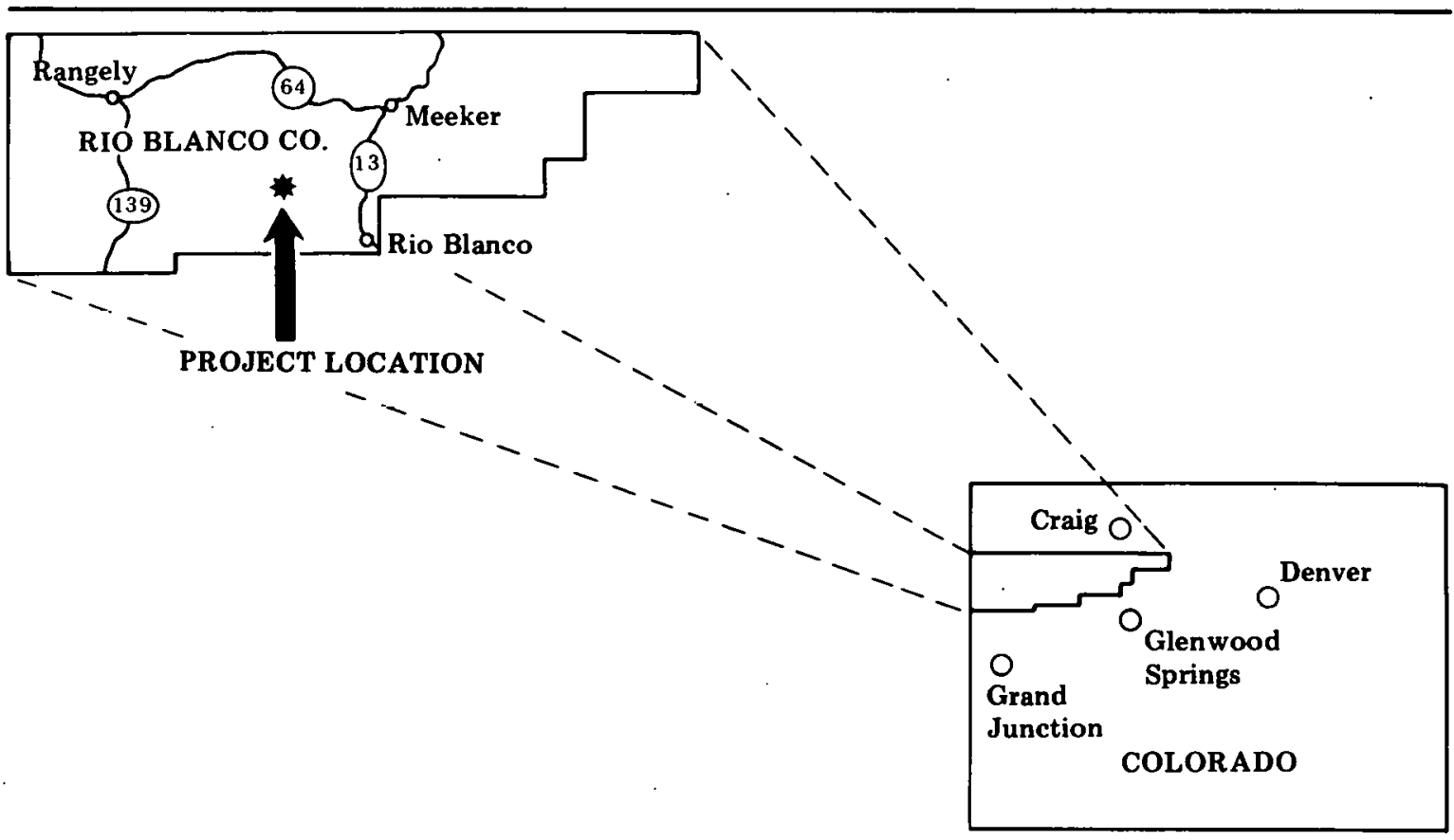




\subsection{RIO BLANCO NATURAL GAS COMPANY}

DOE Contract No. EY-76-C-08-0677 was signed with Rio Blanco Natural Gas Company in June, 1976. The first MHF treatment was performed on October 22, 1976. A supplemental agreement, effective October 1, 1977, provided for a second MHF treatment which was performed on November 30, 1977.

The final report is in preparation. 


\section{NEVADA TEST SITE \\ NYE COUNTY, NEVADA \\ MINEBACK TESTING}

Sandia Laboratories

Status: Active

Albuquerque, New Mexico

Principal Investigator:

D. A. Northrop

\section{OBJECTIVE}

To develop an understanding of the fracturing process for stimulation and thereby improve the production of natural gas from low-permeability reservoirs. This will be accomplished by conducting controlled fracture experiments which are accessible by mineback for direct observation and evaluation.

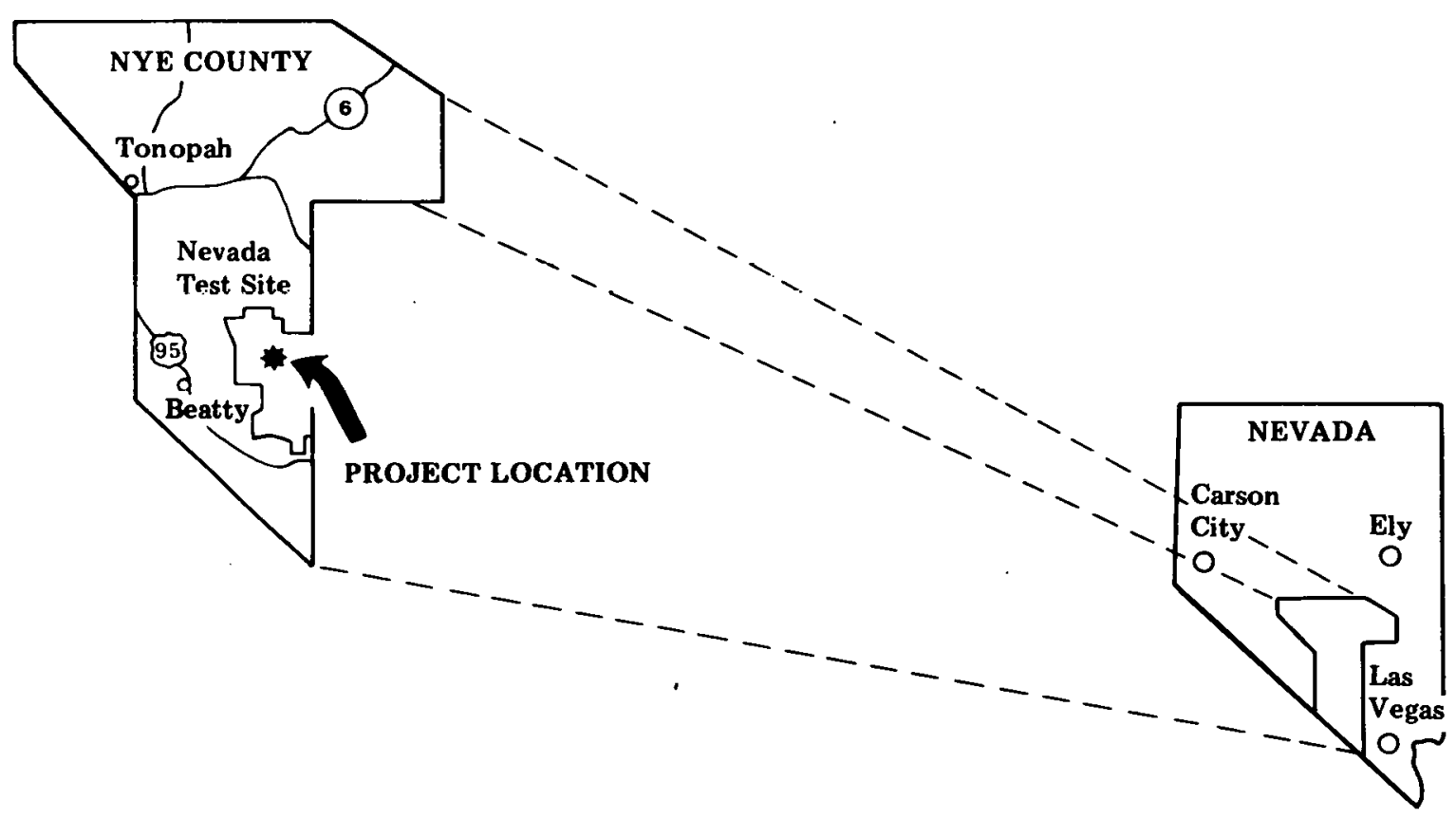




\subsection{SANDIA LABORATORY - MINEBACK}

\subsubsection{Hydraulic Fracture Containment Experiment}

\subsubsection{Hole No. 6 Formation Interface Fracture Experiment}

No additional work was performed on the exploratory coring program to delineate the shape of the Hole No. 6 fractures.

\subsubsection{Interface Test Series}

The Interface Test Series is a continuing in situ test to study hydraulic fracture containment and behavior in the presence of interfaces and in situ stress variations. CFE-3 and the parallel hole, CFE-4, were fractured in August and mineback was initiated in September. A map with the location of these fractures is shown in Figure 5-10. Preliminary data from the CFE-1 and CFE-2, which shows a sharp increase in the minimum principal in situ stress a few feet below the ash-flow tuff/ash-fall tuff interface. However, these fractures were initiated as much as $20 \mathrm{ft}$ below the interface and it appears that there is a second in situ stress anomaly further below the interface. The fractures have been observed to terminate at the approximate location of either of these stress increases.

\subsubsection{Schedule Status}

Figure $5-11$ is a milestone chart depicting Sandia mineback progress. 


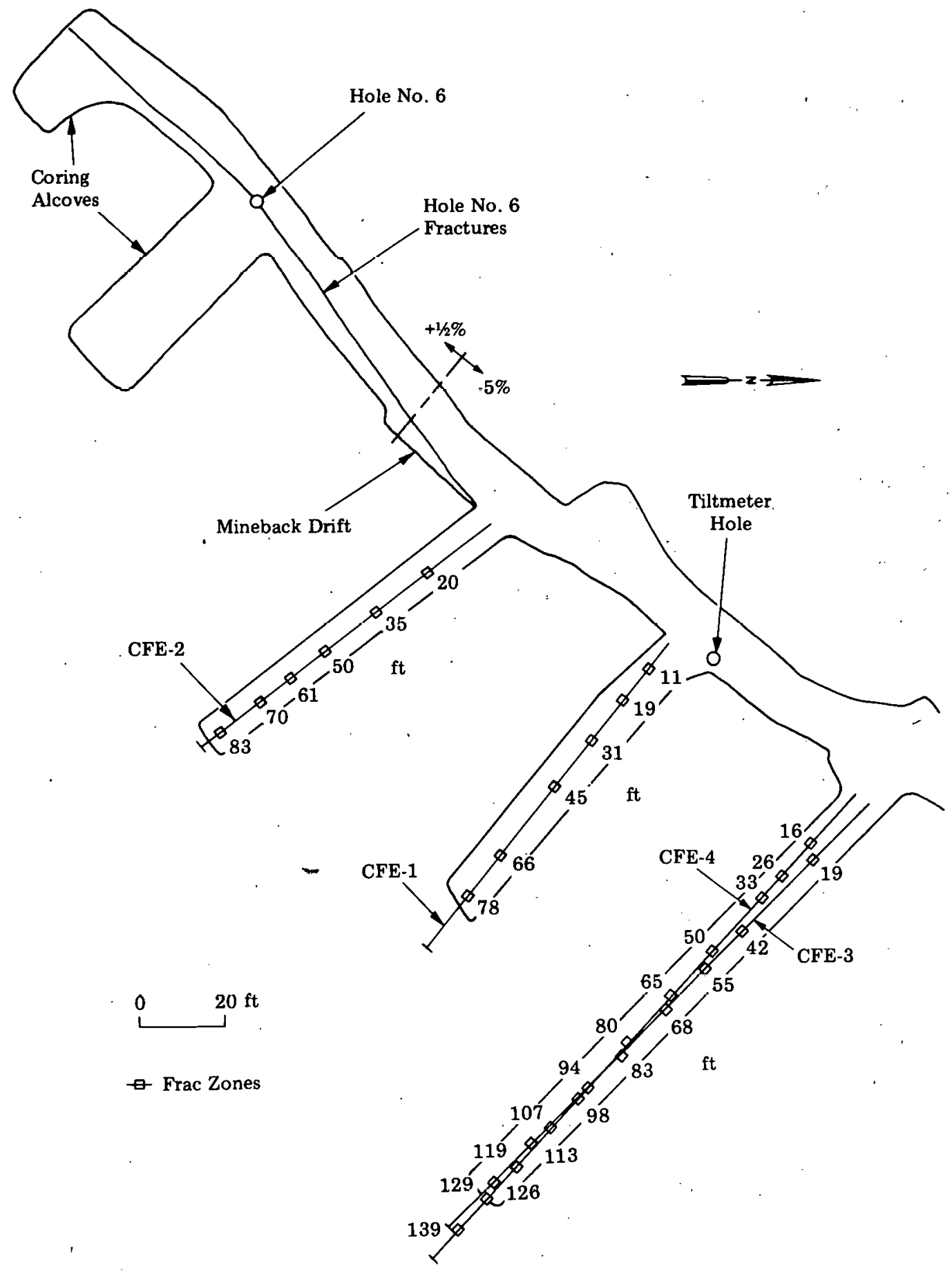

Figure 5-10 Location of CFE Fracture Zones and Mineback 


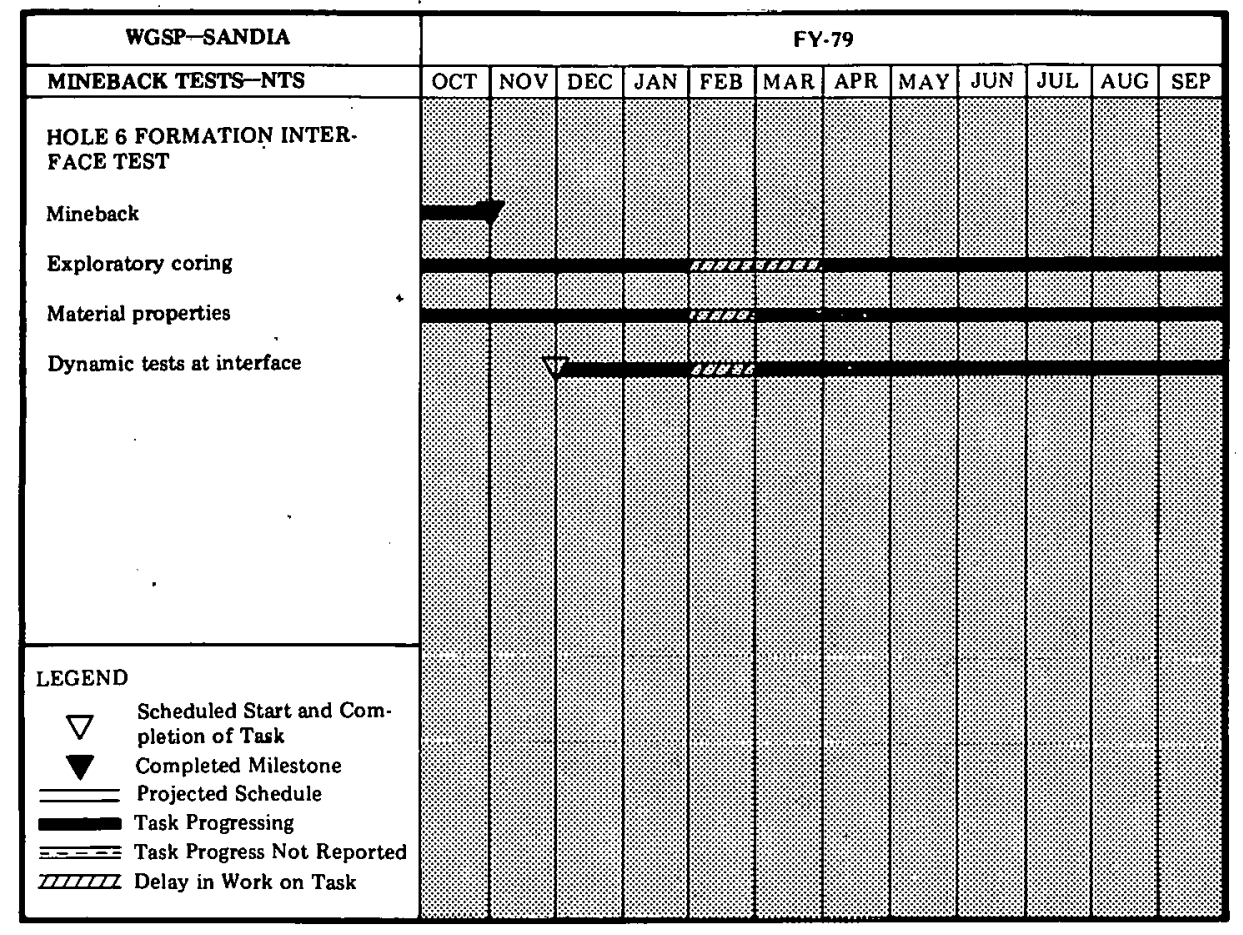

\begin{tabular}{|c|c|c|c|c|c|c|c|c|c|c|c|c|}
\hline WGSP-SANDIA & \multicolumn{12}{|c|}{ FY-79 } \\
\hline MINEBACK TESTS-NTS & OCT & NOV & DEC & JAN & FEB & MAR & APR & MAY & JUN & JUL & AUG & SEP \\
\hline \multicolumn{13}{|l|}{$\begin{array}{l}\text { HOLE } 7 \text { PROPPANT/ } \\
\text { CONDUCTIVITY TEST }\end{array}$} \\
\hline Design test & & & & 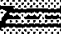 & 13 & $\ldots$ & 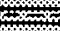 & 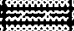 & 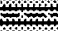 & $=$ & $\ldots$ & $=$ \\
\hline Conduct test & & & & & & & & & & & & \\
\hline Fracture testing & & & & & & & & & & & & \\
\hline Mineback & & & & & & & & & & & & \\
\hline INTERFACE ANALYSES & & & & & & & & & & & & \\
\hline Analytical analyses & & & & & & & & & & & & \\
\hline Numerical (code) analyses & & & & & & & & & & & & \\
\hline Laboratory experimentation & & & & & & & & & & & & \\
\hline & & & & & & & & & & & & \\
\hline & & & & & & & & & & & & \\
\hline & & & & & & & & & & & & \\
\hline & & & & & & & & & & & & \\
\hline
\end{tabular}

Figure 5-11 Milestone Chart-Sandia Mineback 